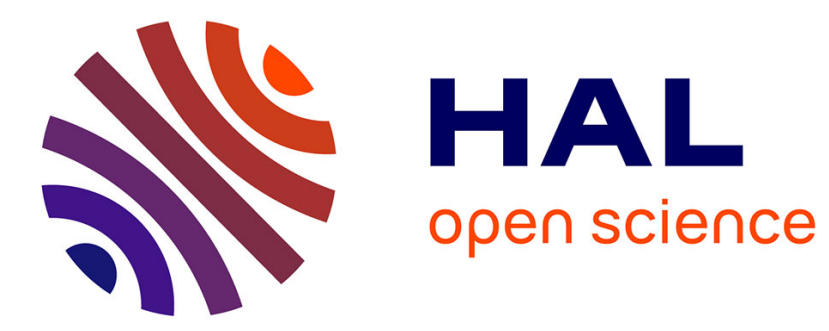

\title{
A computational theory for the production of limb movements
}

Emmanuel Guigon

\section{To cite this version:}

Emmanuel Guigon. A computational theory for the production of limb movements. Psychological Review, 2023, 130 (1), pp.23-51. 10.1037/rev0000323 . hal-03276320v2

\section{HAL Id: hal-03276320 \\ https://hal.science/hal-03276320v2}

Submitted on 19 Jul 2021

HAL is a multi-disciplinary open access archive for the deposit and dissemination of scientific research documents, whether they are published or not. The documents may come from teaching and research institutions in France or abroad, or from public or private research centers.
L'archive ouverte pluridisciplinaire HAL, est destinée au dépôt et à la diffusion de documents scientifiques de niveau recherche, publiés ou non, émanant des établissements d'enseignement et de recherche français ou étrangers, des laboratoires publics ou privés. 


\section{A computational theory for the production of limb movements}

Short title: Optimal motor control

Emmanuel Guigon (https://orcid.org/0000-0002-4506-701X)

Sorbonne Université, CNRS, Institut des Systèmes Intelligents et de Robotique, ISIR, F75005 Paris, France

\section{Corresponding author:}

Emmanuel Guigon

Sorbonne Université, CNRS, Institut des Systèmes Intelligents et de Robotique, ISIR

Pyramide Tour 55 - Boîte Courier 173 - 4 Place Jussieu, 75252 Paris Cedex 05, France

Tel: 33144276382 / Fax: 33144275145 / Email: emmanuel.guigon@sorbonne-universite.fr

Author Note: The ideas in this article are inspired from a preceding article (Guigon et al., 2019), but none of the material presented here has been presented in a symposium or a meeting, nor posted on preprint server. The author declares no competing financial interests.

(C) 2021, American Psychological Association. This paper is not the copy of record and may not exactly replicate the final, authoritative version of the article. Please do not copy or cite without authors' permission. The final article will be available, upon publication, via its DOI:

$10.1037 /$ rev0000323 


\begin{abstract}
Motor control is a fundamental process that underlies all voluntary behavioral responses. Several different theories based on different principles (task dynamics, equilibrium-point theory, passive-motion paradigm, active inference, optimal control) account for specific aspects of how actions are produced, but fail to provide a unified view on this problem. Here we propose a concise theory of motor control based on three principles: optimal feedback control, control with a receding time horizon, and task representation by a series of via-points updated at fixed frequency. By construction, the theory provides a suitable solution to the degrees-of-freedom problem, i.e. trajectory formation in the presence of redundancies and noise. We show through computer simulations that the theory also explains the production of discrete, continuous, rhythmic and temporally-constrained movements, and their parametric and statistical properties (scaling laws, power laws, speed/accuracy tradeoffs). The theory has no free parameters and only limited variations in its implementation details and in the nature of noise are necessary to guarantee its explanatory power.
\end{abstract}

Keywords: modeling, motor control, optimality, variability, time 


\section{Introduction}

Action is the only mean by which the nervous system can communicate evolutions of its internal states to the external world. In any case and irrespective of any theoretical construct, to produce a faint smile, a friendly handshake or a full running pattern, a voluntary (as opposed to a reflex) process should be triggered to create an appropriate temporal pattern of coordination directed to specific muscular groups (Lashley, 1951; Bernstein, 1967). Each and everyone could agree on this statement, yet there is no consensus on the nature of this process (not to say on its anatomical and physiological bases; Arber \& Costa, 2018). Although formal debates on this issue has long since disappeared from the literature (Feldman \& Levin, 1995; Kelso, 1995; Turvey, 1977; but for some recent revivals, see Friston, 2011; Huys, Perdikis, \& Jirsa, 2014; Mohan, Bhat, \& Morasso, 2019), one is still confronted with the same recurring and embarrassing questions when addressing motor control: Are there internal representations in the brain? Does one part of the brain act as a controller which enslaves other parts of the brain and the body? Does the brain manipulate position or force variables? In this framework, a central issue is as follows: how not to keep contemplating and discussing these difficulties, and make progress in the field of motor control modeling that could be beneficial for understanding movement disorders, improving rehabilitation devices or inspiring human-like robotics?

The goal of this article is to describe a computational theory ${ }^{1}$ for the production of limb movement that resolves or circumvents part of these difficulties. The starting point is a recent study of velocity fluctuations during slow movements (Guigon, Chafik, Jarrassé, \& Roby-Brami, 2019). This study shows that a slow movement (with a mean speed below 10

\footnotetext{
${ }^{1}$ The term "computational" is used to designate a class of models based on the theory of control (including optimal control) and internal models (Todorov \& Jordan, 2002; Wolpert \& Ghahramani, 2000). Detailed reading of Todorov and Jordan (2002) is an important prerequisite to understand the present article.
} 
$\mathrm{cm} / \mathrm{s})$ is well described by a time series of constant-duration $(\sim 0.13 \mathrm{~s})$, elementary, discrete displacements whose amplitude is proportional to mean movement speed ${ }^{2}$. The central observation is that the temporal structure of movement kinematics is invariant with changes in movement speed, consistent with the landmark study of Vallbo and Wessberg (1993). The study proposes a model to explain how slow movements are produced: a regular staircase goal position signal at $\sim 8 \mathrm{~Hz}$ is pursued by an optimal feedback controller with a temporal horizon of $0.28 \mathrm{~s}^{3}$. To visualize the process, imagine that you try to reach a virtual target that jumps to a different place every $1 / 8 \mathrm{~s}$ and that your strategy is to assume that at each time the target is stationary and should be reached smoothly in $0.28 \mathrm{~s}$. The resulting movement will be a series of aborted smooth segments of $1 / 8 \mathrm{~s}$ whose velocity is proportional to the size of jumps. The model suggests a general theoretical account of motor control in terms of goals intermittently updated at this frequency and optimally pursued at this horizon. According to the model, any fluctuation observed in a kinematic signal (diversely called submovement, segment, unit, pulse, ...) should be considered a consequence of the pursuit of a temporary goal $^{4}$. Here, a theory is derived on this basis in terms of three computational principles, and is shown to explain a broad range of motor phenomena: trajectory formation in discrete (pointto-point), continuous (drawing, handwriting), rhythmic and temporally-constrained tasks, ubiquity of isochronous behaviors, scaling laws, power laws and speed-accuracy tradeoffs. The article is organized in four parts. First, we outline the context of this study. Next, we

\footnotetext{
${ }^{2}$ An illustrative representation is a regular temporal staircase signal with speed-dependent stair height.

${ }^{3}$ The notions of elementary displacement and temporal horizon are not ambiguous. They emerge from processing and model-based description of experimental data (Guigon et al., $2019)$. Yet the reported values $(0.13 \mathrm{~s}$ for the duration of elementary displacements, $0.28 \mathrm{~s}$ for the temporal horizon) are not universal as they were obtained from data analysis and depend on the choice of a cutoff frequency for low-pass filtering.

4 Important notions related to submovement and intermittency are introduced and comprehensively discussed in Guigon et al. (2019).
} 
present our computational theory of motor control, and a set of simulations that illustrate its main characteristics. Finally, we provide a thorough discussion of the results and the theory.

\section{Context}

\section{The computational approach}

The computational approach to motor control ${ }^{5}$ is prototypically represented by the stochastic optimal feedback control (SOFC) theory of Todorov and Jordan (2002) which has reached a wide audience due to its conciseness, scope and explanatory power (Diedrichsen, Shadmehr, \& Ivry, 2010; Scott, 2004). This theory provides a principled solution to the problem of motor coordination (Bernstein's degrees-of-freedom problem), i.e. how redundant degrees of freedom at each level of the motor hierarchy (from neural space to task space) are coordinated to produce goal-directed actions, and accounts for many characteristics of motor acts (trajectories, structured variability, synergy formation, ...) as by-products of its principles.

SOFC came on top of fifty years of research that elaborated on the application of control, optimal control and optimal estimation theory to the description of human motor behaviors (Baron \& Kleinman, 1969; Flash \& Hogan, 1985; Hatze, 1976; Harris \& Wolpert, 1998; Hoff, 1994; Hogan, 1984; Nelson, 1983; Uno, Kawato, \& Suzuki, 1989; Wolpert, Ghahramani, \& Jordan, 1995). It contained not only a synthesis of previous proposals but also several new ideas on the nature of motor control. First, it abolished the long-held but embarrassing dichotomy between feedforward and feedback control, and replaced it by a more representative control/estimation architecture ${ }^{6}$. Second, it proposed to resolve

\footnotetext{
${ }^{5}$ See Discussion for a presentation of debates on motor control.

${ }^{6}$ In traditional control architectures, a feedforward controller generates an open-loop control signal (e.g. to follow a desired trajectory) and a feedback controller (e.g. a reflex) corrects
} 
redundancy on a moment-by-moment basis and simultaneously for all the available degrees of freedom (from neural space to task space). Third it gave a central, instrumental role to signal-dependent motor noise (multiplicative noise on motor commands; Harris \& Wolpert, 1998; Jones, Hamilton, \& Wolpert, 2002; Todorov, 2002) in the emergence of a "minimum intervention principle" according to which "deviations from the average trajectory are corrected only when they interfere with task performance" (Todorov \& Jordan, 2002). A likely consequence of this principle is the emergence of structured patterns of variability in which a large variability in the contribution of individual degrees of freedom from trial to trial can accompany a low goal-related variability (Bernstein's idea of "repetition without repetition"; concept of uncontrolled manifold; Scholz \& Schöner, 1999).

\section{Limitations}

Despite its successes, SOFC is plagued by several limitations, four of them being quite revealing. First, it comes with a heavy computational burden all the more so that a specific controller must be built for each task at hand. For instance, a via-point experiment (e.g. Experiment 1 in Todorov \& Jordan, 2002) would require a dedicated controller for every possible configuration of the via-points. The burden results from the formulation of the model in terms of a Linear Quadratic Gaussian (LQG) controller in which the task representation is embedded into the cost function. This approach blurs the distinction between skilled and unskilled actions, and leaves no room for multiple, eventually

deviations due to perturbations (Schaal, Ijspeert, \& Billard, 2003). Based on arguments drawn from observations on task variability and goal-directed motor corrections, Todorov and Jordan (2002) rejected these architectures and proposed a control scheme in which a feedforward command is elaborated at each time from feedback information (actual and predicted sensory inputs). The combination of feedforward and feedback control offers no room for open-loop control since control is recalculated at each time based on feedback information. 
suboptimal strategies which can exist to solve a motor task (Ganesh, Haruno, Kawato, \& Burdet, 2010; Kodl, Ganesh, \& Burdet, 2011).

Second, as the task representation is embedded into the cost function, LQG runs several objectives in parallel (e.g. find a solution of lowest energy expenditure possible and the closest possible to a goal), and makes compromises between the objectives. Parameters are needed to weight the different objectives which are not easy to identify experimentally (how one would trade energetic expenditure for accuracy, or positional accuracy for velocity accuracy?), if they ever exist.

Third, as the time to achieve a goal is chosen in advance and decreases gradually as the goal draws nearer, continuous motor behaviors (e.g. tracking, drawing, writing, scribbling, ...) and flexible adjustments following perturbations (Liu \& Todorov, 2007; Shadmehr \& Mussa-Ivaldi, 1994) are not properly explained in this framework. Ad hoc online updating of time is feasible (Liu \& Todorov, 2007), but lacks an underlying principle. In fact, time representation is a deep and ubiquitous issue. Considering again a via-point experiment, not only the overall task duration must be chosen, but also the time to reach each via-point. Experimental studies have shown that the temporal organization of reaching through via-points obeys to an isochrony principle, i.e. the transit time between successive points is almost constant (Flash \& Hogan, 1985; Kodl, Ganesh, \& Burdet, 2011). Isochrony is a venerable concept corresponding to a compensatory regulation of movement speed with amplitude to maintain movement duration approximately constant (Binet \& Courtier, 1893; Bryan, 1892; Denier van der Gon \& Thuring, 1965; Glencross, 1975; Lacquaniti, Terzuolo, \& Viviani, 1983; Viviani \& McCollum, 1983). The origin of isochrony is unknown ${ }^{7}$ and has been rarely addressed in computational studies (Flash \& Hogan, 1985; Flash, Meirovitch, \& Barliya, 2013; Saito, Tsubone, \& Wada, 2006). Incomplete compensation can be explained in

\footnotetext{
${ }^{7}$ Note that isochrony is found in models using classical feedback control and in related models (e.g. Bullock \& Grossberg, 1988).
} 
optimal control models by a cost of time (Harris \& Wolpert, 2006; Hoff, 1994; Shadmehr, Orban de Xivry, Xu-Wilson, \& Shih, 2010). Yet, in these models, complete compensation and strict temporal invariance (isochrony) are irrational and would require an infinite contribution of the cost of time.

Fourth, SOFC (and in fact all optimal control models) produces smooth movements of any duration whereas the smoothness of experimentally recorded movements decreases with increasing movement duration (Salmond, Davidson, \& Charles, 2017; Shmuelof, Krakauer, \& Mazzoni, 2012; review in Guigon et al., 2019). The discrepancy is due to the intrinsically time-invariant nature of optimal trajectories generated by the models. In the same vein, optimal control provides no account for movement intermittency (Doeringer \& Hogan, 1998; Guigon et al., 2019; Vallbo \& Wessberg, 1993), and the constant rate of peak velocity, peak acceleration and peak jerk in motor behaviors (Guigon et al., 2019; Shmuelof et al., 2012; Vallbo \& Wessberg, 1993).

\section{Three computational principles}

Three principles are proposed to exploit the power of the computational approach (Todorov \& Jordan, 2002), and overcome the above-mentioned limitations. They are derived from experimental and theoretical observations on slow movements which suggest that a motor task is represented by a time series of goals updated at a fixed frequency and optimally pursued at a fixed horizon (Guigon et al., 2019). The principles are described in the framework of control theory. Motor control is considered as a control problem in which the behavior of a controlled object is governed by a controller through a control policy and a set of goals to achieve. The 3 principles are the following: 


\section{The control policy is a "universal" optimal feedback control policy}

An optimal feedback control (OFC) policy is a function that takes as input the best estimated state of the controlled object (as given by an optimal state estimator), a goal state and a time to reach the goal, and provides the best control (relative to a cost function) that drives the controlled object to the goal in the given time (Bryson \& Ho, 1975). In the most general (nonlinear, continuous-time) setting, an OFC policy $\boldsymbol{u}(t)$ to reach a goal $\boldsymbol{x}^{G}(t)$ (which is a function of time since it can change at any time) can be formally written at each time $t$

$$
\boldsymbol{u}(t)=\boldsymbol{U}(t),
$$

where $\boldsymbol{U}$ is the function defined in $[t, T(t)]$ by

$$
\boldsymbol{U}\left(t^{\prime}\right)=\operatorname{argmin}_{\boldsymbol{u} \prime} \int_{t}^{T(t)} L\left(\boldsymbol{x}(\theta), \boldsymbol{u}^{\prime}(\theta)\right) d \theta \quad \text { (Equation 1) }
$$

for a dynamics of the controlled object given by

$$
\left.\dot{\boldsymbol{x}}(t)=\boldsymbol{f}(\boldsymbol{x}(t), \boldsymbol{u}(t))+\boldsymbol{n}_{d y n}(t), \quad \text { (Equation } 2\right)
$$

where $\boldsymbol{x}$ is the state of the controlled object ${ }^{8}$ (italic is used for scalars, bold italic for vectors, and bold for matrices, dot is for time derivative), $T$ the time to reach the goal (which is considered a function of time; see below for the choice of $T$ ), $f$ the dynamics of the object, $\boldsymbol{n}_{\boldsymbol{d y n}}$ a noise on the dynamics, and $L$ a cost function. Boundary conditions are given by

$$
\boldsymbol{x}(t)=\widehat{x}(t),
$$

and

$$
\boldsymbol{g}\left(\boldsymbol{x}(T(t)), \boldsymbol{x}^{G}(t)\right)=0, \quad(\text { Equation } 3)
$$

\footnotetext{
${ }^{8}$ The state of the object corresponds to the smallest possible subset of variables that are necessary to describe the behavior of the object (e.g. position, velocity, ...).
} 
where $\boldsymbol{g}$ is a function that specifies the final boundary conditions ${ }^{9}$, and the best estimated state $\widehat{\boldsymbol{x}}$ is defined by

$$
\dot{\hat{\boldsymbol{x}}}(t)=\boldsymbol{f}(\widehat{\boldsymbol{x}}(t), \boldsymbol{u}(t))+\mathbf{K}(t)(\boldsymbol{y}(t)-\mathbf{0} \widehat{\boldsymbol{x}}(t)), \quad(\text { Equation 4) }
$$

where $\mathbf{K}$ is the Kalman gain, $\mathbf{O}$ the observation matrix, and

$$
\boldsymbol{y}(t)=\boldsymbol{0} \boldsymbol{x}(t)+\boldsymbol{n}_{o b s}(t), \quad(\text { Equation } 5)
$$

where $\boldsymbol{n}_{o b s}$ is an observation noise term (see Stengel, 1984 for a mathematical description of optimal estimation and Kalman filtering). Equations 1 to 5 define an architecture that belongs to the class of control/estimation architectures (Figure 1A; Todorov, 2004). The fact that $\boldsymbol{x}^{G}(t)$ is a function of time does not mean that it prescribes a "desired trajectory". The actual trajectory corresponding to $\boldsymbol{x}^{G}(t)$ is not $\boldsymbol{x}^{G}(t)$, but a concatenation of pieces of trajectory produced by the control policy to reach $\boldsymbol{x}^{G}(t)$ at each time $t$.

In this formulation, the control policy (Equation 1) is deterministic and is the same irrespective of the task at hand, i.e. it is aware of the task only through constraints that set the goals (boundary conditions $\boldsymbol{x}^{G}(t)$; Equation 3) to achieve for this task. Such a control policy is called "universal" to indicate that it is a general purpose process independent of any specific task, without any other connotations. In order to justify this choice, we must prove that this formulation has the same explanatory power as the stochastic formulation used by Todorov and Jordan (SOFC), and alleviates some limitations of SOFC.

Figure 1. Building the model. A. Control/estimation architecture corresponding to Equations 1-5. B. Normalized boxcar function. C. Example of a step function. Here, the update times are regularly spaced with step duration $T_{\text {step }}$.

${ }^{9}$ A usual function $\boldsymbol{g}$ is $\boldsymbol{g}\left(\boldsymbol{x}(T(t)), \boldsymbol{x}^{G}(t)\right)=\boldsymbol{x}(T(t))-\boldsymbol{x}^{G}(t)$, but other functions can be used as well. Equation 3 indicates that the goal $\boldsymbol{x}^{G}(t)$ is a goal state, which means that a goal can be possibly specified for every state variable (e.g. position, but also velocity, ...). 
SOFC elaborates a stochastic control policy ${ }^{10}$ which is optimal with respect to the statistics of the noise on the dynamics (Equation 2) and observation (Equation 5), based on

$$
\boldsymbol{U}\left(t^{\prime}\right)=\operatorname{argmin}_{\boldsymbol{u}}\left\langle\int_{t}^{T(t)} L\left(\boldsymbol{x}(\theta), \boldsymbol{u}^{\prime}(\theta), \mathbf{P}(\theta)\right) d \theta\right\rangle
$$

where $\mathbf{P}$ is a set of parameters that specifies the task at hand, and \langle\rangle the mathematical expectation operator over noise. In this framework, the task is represented by an objective (quantity to minimize) rather than by a constraint (condition to fulfill; Equation 3) (Nelson, 1983). Interestingly, the very mechanism which is responsible for the explanatory power of SOFC (Todorov \& Jordan, 2002) is not univoquely related to a formulation as an LQG controller. The only requirements for the occurrence of the minimum intervention principle (see explanation above) are the presence of signal-dependent motor noise and a properly functioning optimal state estimator, irrespective of the stochastic or deterministic nature of the controller, and the formulation in terms of objectives or constraints (Guigon, Baraduc, \& Desmurget, 2008b). More specifically, the fundamental observation that motor variability is organized along redundant task dimensions (Scholz \& Schöner, 1999; Figure 1 in Todorov \& Jordan, 2002) does not necessitate a full-blown formulation in terms of stochastic optimality. The mathematical formulation of SOFC requires a strikingly large number of terms (Equations 2 and 3 in Todorov \& Jordan 2002, Supplementary Notes), but most of these terms could be removed without qualitative consequences on the results reported by Todorov and Jordan (Guigon et al., 2008b). Simulations have shown that a deterministic controller that handles control-dependent objectives and state-dependent, task-related constraints separately and a LQG controller account equally well for structured patterns of motor variability (Guigon et al., 2008b). The main difference between the two approaches is the fact that, in

\footnotetext{
${ }^{10}$ The distinction between stochastic and deterministic processes concerns only the control policy. In all OFC formulations, optimal state estimation is present and stochastic by construction.
} 
the former case, the controller is not stochastic, i.e. it is unaware of the statistics of noise. In fact, it remains to be proven that stochastic optimality is a necessary concept in motor control.

The proposed formulation (Equations 1 to 5) alleviates two limitations of SOFC. First, the computational burden of building a specific control policy for each task at hand is replaced by the burden of building a unique universal policy which is not more complex than any task specific policy ${ }^{11}$. Second, the parameters which are necessary to weight multiple simultaneous objectives in SOFC are absent when constraints are used rather than objectives, which eliminates extra parameters and extra rules to set them.

This discussion might appear uselessly technical. However, as long as principles are concerned, it is important to address the efficiency and conciseness of theoretical constructs. In practice, the two formulations discussed above have the same explanatory power.

\section{The time to reach a goal is constant irrespective of the time already spent for this goal}

Except in highly specific laboratory conditions, neither the world nor the body should be considered as stationary, e.g. due to noise and uncertainties, there is no such thing as a fixed target or fixed posture. Accordingly there is no such thing as the onset, the middle or the end of an action, but only an ongoing state that continuously evolves toward ever changing goals. Temporal flexibility is necessary to account for these facts and is afforded by the function $T(t)$ (Equation 1). The typical choice $T(t)=T_{0}$, where $T_{0}$ is a constant (finite fixed horizon), offers no flexibility as the remaining time to achieve a goal at each time $t$ is $T_{0}-t$ which decreases gradually as time passes and the goal draws nearer. In this framework, different $T_{0}$ are used to obtain movements of different durations which leads to the possibility of smooth

\footnotetext{
${ }^{11}$ The central problem of how an optimal control policy is built and stored by the nervous system remains unsolved.
} 
movements of any duration in contradiction with the fact that movement smoothness decreases with increasing movement duration, and only the fastest movements are likely to be smooth. An alternative choice is $T(t)=\infty$ (infinite horizon formulation; Qian, Jiang, Jiang, \& Mazzoni, 2013; Rigoux \& Guigon, 2012). In this case, movement duration is an emergent characteristic of optimal control, which provides a principled solution to the problem of flexibility. Yet the problem of smoothness remains open. A third choice, retained here as a principle, is $T(t)=t+T_{H}$, where $T_{H}$ is a constant (finite receding horizon), which provides a constant time $T_{H}$ to achieve a goal at each time (Berio, Calinon, \& Fol Leymarie, 2017; Bye \& Neilson, 2008, 2010; Guigon et al., 2019). We use the self-explanatory term receding horizon for $T_{H}^{12}$. In this case, the control policy becomes stationary (independent of time), produces isochronous behaviors, and is intrinsically flexible as any novel goal is automatically pursued at horizon $T_{H}$. Allowing $T_{H}$ to vary would lead to the abovementioned problem of smoothness. The receding horizon is thus considered fixed, not only within a movement, but in fact across all movements and tasks. The value of $T_{H}(0.28 \mathrm{~s})$ was identified in a study of slow movements (Guigon et al., 2019). The most important point is not the value of $T_{H}$ by itself, but the fact that it must be considered a constant rather than an open parameter. How movements of different durations are obtained in the framework of the receding horizon is the object of the third principle (see below).

\section{A task is defined by a sequence of goals played at a fixed and unique frequency}

A strong proposal of Todorov and Jordan is that motor behavior is based on the pursuit of goals rather than tracking of prescribed trajectories. Accordingly, a task can be defined by a discrete sequence of successive, non overlapping goal states (Equation 3), e.g. a sequence of

\footnotetext{
12 The concept of receding horizon belongs to the framework of model predictive control (Garcia, Prett, \& Morari, 1989). No technical information on this framework is needed to understand the model.
} 
via-points (Flash \& Hogan, 1985; Kodl et al., 2011; Experiment 1 in Todorov \& Jordan, 2002; Wada \& Kawato, 1995). In this framework, the most general definition of a task is a time series $\boldsymbol{x}^{G}(t)$ of $N$ goal states $\boldsymbol{x}_{k}^{G}$, updated at times $t_{k}$, given by

$$
\boldsymbol{x}^{G}(t)=\sum_{k=1}^{N-1} \boldsymbol{x}_{k}^{G} \operatorname{boxcar}\left(t, t_{k}, t_{k+1}\right)+\boldsymbol{x}_{N}^{G} \operatorname{boxcar}\left(t, t_{N}, \infty\right) \quad \text { (Equation 6) }
$$

for $t \in\left[t_{1}, \infty[\right.$, where $\operatorname{boxcar}(t, a, b)$ is the function which is 1 for $t \in[a, b]$ and 0 elsewhere (Figure 1B; https://en.wikipedia.org/wiki/Boxcar function). The boxcar function allows a single goal to be selected at each time. We only consider time series with a constant time interval called $T_{\text {step }}$, i.e. $\forall k, t_{k+1}-t_{k}=T_{\text {step }}$. The resulting time series $x^{G}(t)$ is a step function (https://en.wikipedia.org/wiki/Step function), where each step corresponds to a goal that is pursued for a certain time (example in Figure 1C). The goal states are called via-points except the last one which is called target. If any possible task is to be described by Equation 6, a principle should be provided for the choice of goal states and update times (value of $T_{\text {step }}$ ) for a given task. The proposed principle is based on observations on slow movements (Guigon et al., 2019), and is summarized in five rules:

- rule 1: if the task involves state-space constraints (e.g. spatial constraints), the goal states are chosen to match the constraints. For instance, a tracking task would be represented by a series of goals extracted from the trajectory to follow;

- rule 2: if there are no state-space constraints (e.g. scribbling), the goal states are chosen at will;

- rule 3: if the task involves temporal constraints (e.g. follow the beat of metronome), the update times are chosen to match the constraints (e.g. Figure $1 \mathrm{C}$ with $T_{\text {step }}=$ period of the metronome);

- rule 4: if there are no temporal constraints (e.g. scribbling), the goal states are updated with a fixed period $T_{G}=0.13 \mathrm{~s}$, i.e. $t_{k+1}-t_{k}=T_{G}$ (e.g. Figure $1 \mathrm{C}$ with $\left.T_{\text {step }}=T_{G}\right)$. 
- rule 5: the presence of state-space and temporal constraints does not prevent from considering additional goal states updated with period $T_{G}$.

The central element of the proposed principle is in rule 4 which gives a unique recipe to specify the time course of a motor act without a direct specification of its duration. The actual duration is an emergent consequence of the interaction between $T_{G}$ and $T_{H}$, and an increasing function of $N$ (number of goal states). The principle states that $T_{G}$ is the same across all movements and tasks. The value of $T_{G}(0.13 \mathrm{~s})$ was identified in Guigon et al. (2019), to account for velocity fluctuations at $\sim 8 \mathrm{~Hz}$ during slow movements. As for $T_{H}$, the most important point is not the value of $T_{G}$ by itself, but the fact that it must be considered a constant rather than an open parameter (see Table 1 for a summary on time notations).

Note that when the goals are updated with the period $T_{G}$ and pursued at horizon $T_{H}$ (which is longer than $T_{G}$ ), a goal may not be reached before the occurence of the next one.

In the LQG formulation used by Todorov and Jordan, the sequence of goals is integrated in the cost function and the resulting trajectory corresponds to the best way to run through the sequence. In the present approach, the sequence of goals is decided upstream of actual control which allows for multiple, possibly suboptimal task representations.

\section{Simulations}

We present a set of simulations that obey to the three proposed principles. They attempt to reproduce observed characteristics of motor behavior during different tasks. The architecture of the model (Figure 1A) is the same for all the simulations, and the simulations differ only by the sequence of goals used to describe the tasks (Figure 1C). The detailed functioning of the model is the following. A simulation duration $\Delta$ and a timestep of simulation $\delta$ are chosen. At each time $t$ in $[0, \Delta]$, a goal $\boldsymbol{x}^{G}(t)$ and the estimated state $\widehat{\boldsymbol{x}}(t)$ are available and a control policy $\boldsymbol{u}(t)$ is calculated over the interval $\left[t, t+T_{H}\right]$ to reach the current goal $\left(\boldsymbol{x}^{G}(t)\right)$ 
at time $t+T_{H}$ starting from the estimated state at time $t$ (Equations 1 and 3). Then the new state $\boldsymbol{x}(t+\delta)$ and new estimated state $\widehat{x}(t+\delta)$ at time $t+\delta$ due to the control, observation and noise at time $t$ are calculated from current state $\boldsymbol{x}(t)$ and current estimated state $\widehat{\boldsymbol{x}}(t)$ (Equations 2, 4 and 5). The process is repeated at time $t+\delta$ and stopped when current time is $\Delta$. Note that the simulation duration $\Delta$ defines the period of time during which the behavior of the model is simulated and has no influence on this behavior. $\Delta$ is chosen to cover the duration of the sequence of goals (see Table 1 for a summary on time notations).

The principles do not specify a unique model. Some freedom remains on how sequences of goals are built (in allocentric or egocentric coordinates) and on the nature of constraints at each goal (e.g. position, velocity, force, ...). The possible variations of the model are systematically tested.

There are no free parameters in the deterministic simulations and only noise parameters in the stochastic simulations. The noise parameters are given with no justification (the noise parameters not specified are 0), but a specific section (the last one) is dedicated to the study of noise and variability.

\section{Methods}

\section{One-dimensional inertial point}

The three computational principles were applied to an inertial point actuated by a linear muscle in the presence of noise, with a quadratic cost function, and a Kalman filter for optimal state estimation. The rationale for this choice (in particular the absence of redundancy) is the following. As the model inherits properties from the model of Todorov and Jordan (2002), we deem it not necessary to address issues already considered in their work (kinematic redundancy, muscular redundancy, formation of uncontrolled manifolds and synergies). 
The dynamics of the point was given by Equation 2, with the state vector $\boldsymbol{x}=$ [ $p$ v $\quad a e$ ] ( $p$ position, $v$ velocity, a muscular activation, $e$ muscular excitation), the control vector $\boldsymbol{u}=[u]$, and the vectorial function $\boldsymbol{f}$ defined by

$$
\left\{\begin{array}{c}
\dot{p}=v \\
m \dot{v}=a \\
\tau \dot{a}=-a+e \\
\tau \dot{e}=-e+u
\end{array}\right.
$$

where $m$ is the mass of the point and $\tau$ the muscle time constant. This formulation corresponds to a linear, second-order (Newtonian) dynamics coupled to a linear, second-order (low-pass filtering) force generator, and widely used in motor control models (Harris \& Wolpert, 1998; Todorov \& Jordan, 2002; van der Helm \& Rozendaal, 2000).

The cost function was defined by

$$
L(\boldsymbol{x}, \boldsymbol{u})=u^{2} .
$$

State estimation obeyed to Equation 4 and observation to Equation 5. The Kalman gain was calculated following Guigon et al. (2008b) for sources of noise described below.

\section{Task representation: series of goals and boundary conditions}

A task was represented by a time series of goal states (Equation 6). There are two ways to define a sequence. The next goal can be defined relative to the current goal or relative to the current (estimated) state of the system. It corresponds broadly to the distinction between allocentric and egocentric coding of goals. Thus we considered an absolute goal setting policy when

$$
\boldsymbol{x}_{k}^{G}=\boldsymbol{x}_{k-1}^{G}+\boldsymbol{\alpha}_{k} \quad(\text { Equation 7) }
$$

and a relative goal setting policy when

$$
\boldsymbol{x}_{k}^{G}=\widehat{\boldsymbol{x}}\left(t_{k}\right)+\boldsymbol{\alpha}_{k}, \quad(\text { Equation 8) }
$$

where $\boldsymbol{\alpha}_{k}$ is an arbitrary sequence. 
In the most general setting, final boundary conditions were defined by Equation 3. The choice of function $\boldsymbol{g}$ is a fundamental issue. For instance, different boundary conditions should probably be used for discrete and continuous movements, e.g. it may not be necessary or effective to impose a zero-velocity constraint at a via-point in a continuous movement. Yet there is no self-evident principle for the choice of $\boldsymbol{g}$ (e.g. what is an appropriate velocity constraint at a via-point in a continuous movement?), and it is necessary to consider and test different possibilities. There are two aspects in the construction of boundary conditions. First, the states to be constrained are chosen, the remaining unconstrained states being automatically determined by the optimal control process. For a 4 dimensional problem (position, velocity, activation, excitation), there are 15 different configurations, but only three were considered:

- the full-state constraint, i.e.

$$
\left\{\begin{array}{l}
p\left(t+T_{H}\right)-p^{G}(t)=0 \\
v\left(t+T_{H}\right)-v^{G}(t)=0 \\
a\left(t+T_{H}\right)-a^{G}(t)=0 \\
e\left(t+T_{H}\right)-e^{G}(t)=0
\end{array}\right.
$$

- the partial-position constraint, i.e.

$$
p\left(t+T_{H}\right)-p^{G}(t)=0 \quad(\text { Equation } 9)
$$

- the partial-position/velocity constraint, i.e.

$$
\left\{\begin{array}{l}
p\left(t+T_{H}\right)-p^{G}(t)=0 \\
v\left(t+T_{H}\right)-v^{G}(t)=0
\end{array}\right. \text { (Equation 10) }
$$

Second, in the full-state constraint, the desired values of the constrained states are chosen. It is in general easy to set positional constraints (e.g. positions of via-points) and sometimes possible to set velocity constraints (e.g. stationary via-points). For the other states, two conservative methods were considered:

- a zero-value method (the states go to 0), e.g. when only the position goal is known 


$$
\left\{\begin{array}{c}
p\left(t+T_{H}\right)-p^{G}(t)=0 \\
v\left(t+T_{H}\right)=0 \\
a\left(t+T_{H}\right)=0 \\
e\left(t+T_{H}\right)=0
\end{array}\right.
$$

or when the position and velocity goals are known

$$
\left\{\begin{array}{c}
p\left(t+T_{H}\right)-p^{G}(t)=0 \\
v\left(t+T_{H}\right)-v^{G}(t)=0 \\
a\left(t+T_{H}\right)=0 \\
e\left(t+T_{H}\right)=0
\end{array}\right.
$$

- a current-value method (the states keep their current value), e.g. when only the position goal is known

$$
\left\{\begin{array}{c}
p\left(t+T_{H}\right)-p^{G}(t)=0 \\
v\left(t+T_{H}\right)-v(t)=0 \\
a\left(t+T_{H}\right)-a(t)=0 \\
e\left(t+T_{H}\right)-e(t)=0
\end{array}\right. \text { (Equation 13) }
$$

or when the position and velocity goals are known

$$
\left\{\begin{array}{c}
p\left(t+T_{H}\right)-p^{G}(t)=0 \\
v\left(t+T_{H}\right)-v^{G}(t)=0 \\
a\left(t+T_{H}\right)-a(t)=0 \\
e\left(t+T_{H}\right)-e(t)=0
\end{array}\right. \text { (Equation 14) }
$$

This description of boundary constraints applied to the via-points (goal states 1 to $N-1$; Equation 6). The target (goal state $N$; Equation 6) was considered as stationary and always obeyed to Equation 11.

In summary, a task representation was defined by a series of goal, a goal setting policy (absolute or relative), a boundary constraint (full-state, partial-position, partialposition/velocity) and a boundary method for the full-state constraint (zero- or current-value). It should be noticed that the full-state constraint defined by Equation 11 or Equation 13 is not similar to the partial-position constraint (Equation 9). In the latter case, the values of velocity, activation, and excitation are constrained by optimization. For a similar reason, the full-state 
constraint defined by Equation 12 or Equation 14 is not similar to the partial-position/velocity constraint (Equation 10).

The apparent complexity in the construction of task representations is due to the fact that there are many implementation details and we deem it necessary to evaluate their contribution to the functioning of the model.

\section{Noise}

In the proposed framework, variability is modeled by the presence of white noise sources. We assumed that every process is possibly corrupted by noise and noise can be described by the standard deviation $(\sigma)$ of a Gaussian variable. The dynamics (subscript $m$ for motor; Equation 2) and observation (subscript $s$ for sensory; Equation 5) noises were described in terms of signal-independent (SIN) and signal-dependent (SDN) terms $\left(\operatorname{SIN}_{m}, \sigma^{\xi} ; S D N_{m}, \sigma^{\varepsilon}\right.$; $S I N_{s}, \sigma^{\omega} ; S D N_{S}, \sigma^{\epsilon}$; Guigon et al., 2008b; Todorov, 2005). Other sources of noise were considered for:

- $T_{H}$, i.e. initially and at every $T_{G}$, the nominal receding horizon $T_{H}$ became $\left(1+\sigma^{\zeta} \zeta\right) T_{H}$, where $\zeta$ is a realization of a standard normal variable and $\sigma^{\zeta}$ a parameter;

- $T_{G}$, i.e. at every $T_{G}$, the nominal goal time $T_{G}$ became $\left(1+\sigma^{\gamma} \gamma\right) T_{G}$, where $\gamma$ is a realization of a standard normal variable and $\sigma^{\gamma}$ a parameter;

- $p^{G}$, i.e. initially the nominal position goal $p^{G}$ became $\left(1+\sigma^{\chi} \chi\right) p^{G}$, where $\chi$ is a realization of a standard normal variable and $\sigma^{\chi}$ a parameter.

The dynamics and observation noises were termed execution noises as they act continuously during the movement. The other noises were termed planning noises as they act before the movement and possibly discretely during the movement (at each $T_{G}$ ). 


\section{Parameters}

Parameters were $m=1 \mathrm{~kg}, \tau=0.05 \mathrm{~s}, T_{H}=0.28 \mathrm{~s}, T_{G}=0.13 \mathrm{~s}$, and $\mathbf{0}$ was the $4 \times 4$ identity matrix. For calculating the Kalman gain, the ratio $\sigma^{\xi} / \sigma^{\omega}$ was 0.001 .

\section{Solutions}

The optimal feedback control policy and the Kalman gain were calculated analytically as described in Guigon et al. (2008b), and simulated numerically with the time step $\delta=$ $0.001 \mathrm{~s}$. A complete mathematical background is given in the Online Supplemental Material.

\section{Two-dimensional inertial point}

To simulate drawing movements, independent inertial points moving along perpendicular directions in the plane were considered (same formulation, same parameters).

\section{Data analysis}

Simulations produced time series of position that were analyzed to determine movement characteristics. Movement duration was defined by the time between the beginning of the simulation and the end of the movement obtained by a velocity threshold $(0.05 \mathrm{~m} / \mathrm{s})$. Endpoint variability was defined as the standard deviation of the position at the end of the movement. Timing variability was defined as the standard deviation of movement duration.

\section{Fastest point-to-point movements: smoothness and isochrony}

The fastest point-to-point movement was obtained when, starting from initial state $\boldsymbol{x}_{0}=\left[\begin{array}{llll}0 & 0 & 0 & 0\end{array}\right]$, a stationary target goal $\boldsymbol{x}^{G}=\left[\begin{array}{llll}p & 0 & 0 & 0\end{array}\right],(p$ is the prescribed movement amplitude; full-state constraint; Equation 11) was set at time $t=0$ and pursued at horizon $T_{H}$ (Figure 2A, dotted lines). The resulting trajectory was regular (Figure 2A) with a bell-shaped velocity profile (Figure 1B). As movement amplitude increased, peak velocity increased and movement duration remained constant (Figure 2B). These movements correspond to a unique 
class of smooth movements with biphasic acceleration profiles (Figure 2B, inset). They were close to minimum-jerk movements, with a jerk ratio of 1.5 (ratio between integrated jerk and jerk of the corresponding minimum-jerk trajectory). Note that the goal time $T_{G}$ played no role in these simulations since there were no via-points.

Two points should be noticed. First, by construction a movement obtained by control with a receding horizon never really stops in the sense that there always remains the same time to complete a movement irrespective of the remaining distance to the goal. In practice, movement duration can be properly defined by setting a threshold below which velocity fluctuations are negligible. The same consideration also applies to experimental recordings. Second, humans can produce movements that are faster than those described here $(\sim 0.4 \mathrm{~s}$ vs $\sim 0.2 \mathrm{~s}$ in Hoffman \& Strick, 1986). Much faster displacements $(\sim 0.2 \mathrm{~s})$ can be obtained in the model in the framework of rhythmic movements (see Figure 12). The movements described by Hoffman and Strick (1986) have large overshoots and terminal oscillations, and could be considered as aborted rhythmic movements.

Figure 2. Simulation of the fastest point-to-point movements. A. Position profiles. Amplitude of $0.1 \mathrm{~m}$ (black), $0.2 \mathrm{~m}$ (red), $0.3 \mathrm{~m}$ (green), $0.4 \mathrm{~m}$ (blue). Dotted lines show task representation. B. Velocity profiles. Inset: acceleration profiles.

\section{Slow point-to-point movements: segmentation}

A slow movement of mean speed $s$ was obtained with the full-state constraint, either the zero-value (Equation 12) or the current-value (Equation 14) method, and the absolute goal setting policy (Equation 7). The time series of goal states was built according to rules 1 and 4: goal states were chosen along the desired spatial path of the movement and updated with period $T_{G}$. Starting from initial state $\boldsymbol{x}_{0}=\left[\begin{array}{llll}0 & 0 & 0 & 0\end{array}\right]$, the first goal state was $\boldsymbol{x}_{1}^{G}=\left[\begin{array}{lll}s T_{G} & s & 0\end{array}\right]$, and the following goal states were given by Equation 7 with $\boldsymbol{\alpha}_{k}=\left[\begin{array}{llll}s T_{G} & 0 & 0 & 0\end{array}\right]$. Accordingly, 
the position constraint was a staircase temporal signal of base $T_{G}$ and height $s T_{G}$ (dotted lines; Figure 3A), and the velocity constraint a constant signal of size $s$ (dotted lines; Figure 3B). The simulated position and velocity profiles are shown in Figure 3 (plain lines) for 4 mean speeds. We showed previously that this model provides an accurate account of properties of slow movements (Guigon et al., 2019). Note that these movements can be terminated by setting a stationary target goal (Equation 6).

The relative goal setting policy (Equation 8) lead to slow movements which did not comply with the required mean speed. The partial-position/velocity constraint (Equation 10) lead to slow movements with smaller velocity fluctuations. As the value of $T_{H}$ was derived from the size of these fluctuations (Guigon et al., 2019), it raises the possibility for a lower value of $T_{H}$. Yet an independent cross-check of the value of $T_{H}$ was obtained in the study of drawing movements (see below).

Figure 3. Simulation of slow point-to-point movements. A. Position profiles. Mean speed of $0.025 \mathrm{~m} / \mathrm{s}$ (black), $0.05 \mathrm{~m} / \mathrm{s}$ (red), $0.075 \mathrm{~m} / \mathrm{s}$ (green), $0.1 \mathrm{~m} / \mathrm{s}$ (blue). Simulations with full-state constraint, zero-value method, and absolute goal setting policy. B. Velocity profiles. Dotted lines show task representation in position and velocity.

\section{Point-to-point movements of intermediate duration}

Point-to-point movements that are neither very fast nor very slow have been ubiquitously reported in the literature. For instance, the mean movement speed in the original data of Fitts (1954) was in the range 10-100 cm/s (amplitude 5-40 cm, duration 0.18-0.73 s). The velocity profile of such movements is more or less irregular and asymmetric with one or more peaks (Darling, Cole, \& Abbs, 1988; Morasso, Mussa-Ivaldi, \& Ruggiero, 1983; Salmond et al., 2017). Following the proposed principles, the duration of a movement is related to the number and position of via-points used to define it. There is a priori an infinite number of ways to set via-points. In keeping with the study of slow movements, we considered 
movements built using via-points specified by a fraction of movement amplitude (rules 1, 2 and 4). For a movement of amplitude $A$ and fraction $f$ (in $[0 ; 1]$ ), we took $\alpha_{k}=f A$ to define the position of the kth via-point (Equation 7 or Equation 8). A via-point whose position exceeded $A$ was set at $A$, and considered as a target (Equation 6). Simulations were run for one amplitude and 10 fractions (between 0.3 and 1), with full-state constraint and zero-value method (Equation 11), for absolute (Equation 7; Figure 4A) and relative (Equation 8; Figure 4D) goal setting policy.

For the absolute goal setting policy, the via-points were equally distributed along the movements (Figure 4A), and the trajectories were regular (Figure 4B) with velocity profiles that became more irregular as movement duration increased (Figure 4C). The fastest movement was identical to those described in Figure 2 and the slowest movement was similar to those described in Figure 3. For the relative goal setting policy, the distribution of viapoints along the movements was time-dependent (in fact, speed-dependent; Figure 4D), and the trajectories were as described for the absolute goal setting policy (Figure 4E,F). The main quantitative difference between the two goal setting policies is observed on the time to peak velocity which scales with duration in one case (Figure 4C), but not in the other (Figure 4F). This difference will be important in the study of scaling laws and Fitts' laws (see below).

Figure 4. Simulation of movements of intermediate duration. A. Position of viapoints (plain square) for 10 movements of the same amplitude (diamond: start point; circle: target) with the absolute goal setting policy. B. Trajectories of the movements described in A. C. Velocity profiles of the movements described in A. D. Same as A the relative goal setting policy. E. Trajectories of the movements described in D. F. Velocity profiles of the movements described in $\mathbf{D}$.

\section{Drawing movements: isochrony and power laws}

A shape was described by a sequence of appropriately placed via-points updated at period $T_{G}$ (rules 2 and 4). For instance, four points at the vertex of a square presented sequentially at 
interval $T_{G}$ generated a circle figure (partial-position constraint, Equation 9; absolute goal setting policy, Equation 7; Figure 5A). The corresponding times serie of positional goals is shown in Figure 5B. No velocity goals were imposed at the via-points. Tangential velocity was inversely correlated with curvature and cycle duration was constant (Figure 5E). A more precise circle drawing was obtained with an 8-points sequence (Figure 5C,D). Drawing had similar scaling properties, but was slower (Figure 5E). Cycle duration increased linearly with the number of via-points (Figure 5E, inset).

For the same via-points, the partial-position/velocity (Equation 10) and the full-state constraints (Equations 11-14) lead to polygons rather than circles. The relative goal setting policy (Equation 8) was not appropriate to obtain a specific geometric shape.

Figure 5. Drawing circles. A. A 10 -cm radius circle defined by 4 via-points
(colored squares; color code from left to right on the colorbar), 30 turns,
counterclockwise rotation. Mean cycle duration was $0.52 \mathrm{~s}$. Calibration is $10 \mathrm{~cm}$.
B. Time series of positional goals in $\mathrm{x}$ (thick) and y (thin) for A. C. A $10-\mathrm{cm}$
radius circle defined by 8 via-points (colored diamonds), 30 turns,
counterclockwise rotation. Mean cycle duration was $1.04 \mathrm{~s}$. D. Time series of
positional goals in $\mathrm{x}($ thick) and $\mathrm{y}($ thin $)$ for C. E. Relationship
curvature/tangential velocity (left axis, closed symbols) and curvature/cycle
duration (right axis, open symbols). Squares correspond to 4 via-points, diamond
to 8 via-points. Dashed line from Viviani and McCollum (1983), fig. 3A. Inset:
relationship between the number of via-points and cycle duration. Simulations
with partial-position constraint, absolute goal setting policy, in the presence of
$S D N_{m}\left(\sigma^{\varepsilon}=1\right)$.

Studies of drawing curved shapes revealed a simple power law relationship

$$
V(t) \propto C(t)^{-\beta}
$$

between tangential velocity $V$ and curvature $C$ with $\beta \approx 1 / 3$ (Lacquaniti, Terzuolo, \& Viviani, 1983). An ellipse defined by the four vertices of a rectangle (with an aspect ratio of 2) is shown in Figure 6A and complies with the one-third power law (Figure 6B).

Huh and Sejnowski (2015) showed that there exists a continuum of power laws related to the frequency content of shapes (see also Zago, Matic, Flash, Gomez-Marin, \& 
Lacquaniti, 2018). We built a via-point representation of 12 shapes (rules 2 and 4) corresponding to frequencies $v=2 / 33,2 / 5,3 / 5,2 / 3,4 / 5,4 / 3,3 / 2,2$ (ellipse), 5/2, 3, 4, 5 according to the equation

$$
\log (r)=\mu \sin (v \theta)
$$

in polar coordinates $(r, \theta), \mu$ is a parameter (chosen to obtain the shapes shown in Huh and Sejnowski, fig. 4). The exponent $\beta$ of the power law varied with frequency with a trend close to that reported experimentally (Figure 6C). The one-third power law was observed only for ellipses. A parametric analysis of these results shows that the chosen values of $T_{H}$ and $T_{G}$ provide the best account of experimental data over the range [0.18-0.48] for $T_{H}$ and [0.130.23] for $T_{G}$ (Appendix A, Figure A1).

Figure 6. Power laws. A. Ellipse defined by 4 via-points, 40 turns, counterclockwise rotation, arbitrary dimension. Same conventions as in Figure 5A. B. Instantaneous relationship between tangential velocity and curvature for the ellipse in $\mathbf{A} . \beta=0.328\left(R^{2}=0.98\right)$. C. Relationship between frequency content $v$ and power law exponent $\beta$ for 12 shapes. Dotted line from Huh and Sejnowski (2015), dashed line from Zago et al. (2018). Horizontal and vertical plain lines indicate the case of an ellipse $(v=2, \beta \approx 1 / 3)$. Simulations with partial-position constraint, absolute goal setting policy, in the presence of $\operatorname{SDN}_{m}\left(\sigma^{\varepsilon}=1\right)$.

\section{Handwriting}

The word "flow" was used for comparison with Huys et al. (2014). It was described as a time series of 21 via-points (Figure 7A): the letter $f$ was represented by two symmetrical rectangular triangles (dark blue), the letter $l$ by an isocele triangle (light blue), the letter $o$ by a smaller triangle (green) and the letter $w$ by a kind of $w$ (yellow to red). The methods were as described for drawing. The writing was smooth (Figure 7B), made of strokes (Figure 7C), and isochronous (by construction), consistent with experimental observations (Denier van der Gon, \& Thuring, 1965; Hollerbach, 1981; Viviani \& Terzuolo, 1982). This simulation is 
provided here for illustrative purpose. The via-points were chosen by hand. No general recipe for building word representations was searched for. In fact, there are no specific characteristics of handwriting that could be used to challenge the theory.

Figure 7. Writing the word "flow". A. Via-point representation of the word. The via-points are shown as nodes of a graph connected by colored edges. The order is indicated by the color gradient. $\mathbf{B}$. Written word. The colors correspond to the via-point representation. C. Velocity profile. No spatial unit is necessary since writing is isochronous. Simulation with partial-position constraint, absolute goal setting policy, in the absence of noise.

\section{Scaling laws}

Scaling laws correspond to ubiquitous relationships between movement characteristics (e.g. amplitude and duration; Fitts, 1954; Gordon, Ghilardi, Cooper, \& Ghez, 1994). For instance, the fastest point-to-point movements of Figure 2 have constant duration and time to peak velocity, and linearly increasing peak velocity and peak acceleration with amplitude (Figure 8A). We call this pattern an isochronous scaling law. To analyze the diversity of scaling laws, we considered movements built as described for movements of intermediate duration (Figure 4A,D). Simulations were run for 4 amplitudes and 10 fractions (between 0.5 and 1), with full-state constraint and zero-value method (Equation 11), for absolute (Equation 7; Figure 8B) and relative (Equation 8; Figure 8C) goal setting policy. For $f<1$, there was one via-point for absolute goal setting policy (Appendix B, Figure B1A), and one or two via-points for relative goal setting policy (Figure B1B). We observed that each fraction $f$ corresponded to a specific isochronous scaling law (one color; Figure 8B,C). For each amplitude, movement duration increased and peak velocity/acceleration decreased as $f$ decreased. As already observed (Figure 4C,F), the two goal setting policies differed on the time to peak velocity: it scaled with $f$ for absolute goal setting policy and was constant for 
relative goal setting policy (Figure $8 \mathrm{~B}, \mathrm{C}$ ). The scaling laws remained similar for the currentvalue method (Equation 13).

Figure 8. Scaling laws. A. Scaling laws for the fastest point-point movements (data from Figure 2). (circle) movement duration, left side axis; (square) time to peak velocity, right side axis. B. A family of isochronous scaling law obtained with full-state constraint, zero-value method and absolute goal setting policy. Each point corresponds to a movement of a given amplitude and a specific position of via-points. Each color corresponds to four movements (amplitudes $0.1,0.2,0.3,0.4 \mathrm{~m}$ ) with a specific fraction (10 fractions between 0.5 and 1$)$ of amplitude between the via-points. For instance a fraction of 0.6 means that the four movements have one via-point at $0.06,0.12,0.18$ and $0.24 \mathrm{~m}$, respectively. C. Same as $\mathbf{B}$ with relative goal setting policy.

From a family of isochronous scaling laws (Figure 8B,C), different types of scaling strategies could be obtained (Figure 9):

- via-points at a decreasing fraction of amplitude with absolute goal setting policy gave movements with increasing duration, time to peak velocity, peak velocity, and peak acceleration (Figure 9A; Gordon et al., 1994);

- via-points at a fixed distance with absolute goal setting policy gave movements with increasing duration, peak velocity and time to peak velocity, but constant peak acceleration (Figure 8B; non-dominant strategy in Sainburg \& Schaeffer, 2004);

- via-points at a decreasing fraction of amplitude with relative goal setting policy gave movements with increasing duration, peak velocity and peak acceleration, but constant time to peak velocity (Figure 9C; dominant strategy in Sainburg \& Schaeffer, 2004).

The model can thus account for the fact that the kinematic landmarks of a movement do not systematically scale with changes in movement duration (Baraduc, Thobois, Gan, Broussolle, \& Desmurget, 2013; Sainburg \& Schaeffer, 2004; Sarlegna, Blouin, Bresciani, Bourdin, Vercher, \& Gauthier, 2003; Torres \& Andersen, 2006; Worringham, 1991). The 
model does not provide a rationale for the different strategies, but this issue will become clearer in the framework of Fitts' law (see below).

Figure 9. Three scaling strategies. A. Absolute goal setting policy, via-points at fraction $0.9,0.8,0.7$ and 0.6 , for amplitude $0.1,0.2,0.3,0.4 \mathrm{~m}$, respectively. B. Absolute goal setting policy, via-points at $0.11 \mathrm{~m}$ for all amplitudes. C. Relative goal setting policy, via-points at fraction $0.9,0.8,0.7$ and 0.6, respectively. Same conventions as in Figure 8. Below each scaling strategie, the position of the via-points for each amplitude is shown (same format as in Figure 4A,D).

\section{Discrete and rhythmic movements}

There exists a classical distinction between discrete and rhythmic movements (Guiard, 1993; Hogan \& Sternad, 2007). An open question is whether the model can account for this distinction. To produce a rhythmic behavior, we considered a sequence of two alternating position goals (distance $0.2 \mathrm{~m}$ ) at a fixed frequency (corresponding to an internally generated periodic signal) as a task representation (rules 1 and 3), with partial-position constraint (Equation 9). At $1.5 \mathrm{~Hz}$, position, velocity and acceleration traces were almost sinusoidal (Figure 10A). There were no dwell periods (i.e. periods of near-zero velocity) and harmonicity (defined as the ratio between minimum and maximum acceleration; Guiard, 1993) was close to one. At $0.7 \mathrm{~Hz}$, kinematics was much more irregular with consistent dwell periods and near zero harmonicity (Figure 10B). Dwell time and amplitude decreased with frequency (Figure 10C), and harmonicity increased with frequency (Figure 10D). Qualitatively, nonzero dwell times were found for frequencies below $1 \mathrm{~Hz}$, i.e. for unidirectional displacements longer than $0.5 \mathrm{~s}$, consistent with Sternad, Marino, Charles, Duarte, Dipietro, \& Hogan (2013). The corresponding movements are considered as discrete (Hogan \& Sternad, 2007). We note that movements of such durations ( $>0.5 \mathrm{~s})$ could be produced using via-points (see Figure 8; rule 5) and would not encompass a dwell time. Using either the partial-position/velocity or the full-state constraint lead to qualitatively 
similar results at high frequencies and a shift to nonzero dwell times around $2 \mathrm{~Hz}$. These results do not depend on the goal time $T_{G}$ since the goals were updated at imposed frequencies.

These results show that the distinction between discrete and rhythmic movements could correspond to a change in behavior (appearance of dwell periods) with required movement frequency of one and the same control process. Further analysis of rhythmic movements is given in Appendix C.

Figure 10. Rhythmic movements. A. (top) Position and acceleration (bottom) Velocity. Vertical dashed and dotted lines delimit dwell periods (velocity threshold at $0.01 \mathrm{~m} / \mathrm{s}$ ). Frequency was $1.5 \mathrm{~Hz}$. Dwell time was $0.015 \mathrm{~s}$. Harmonicity was 0.67 . B. Same as A for a frequency of $0.7 \mathrm{~Hz}$. Dwell time was $0.172 \mathrm{~s}$. Harmonicity was 0.28 . C. Mean dwell time as a function of frequency. Inset: mean movement amplitude as a function of frequency. (gray circle) $1.5 \mathrm{~Hz}$; (black circle) $0.7 \mathrm{~Hz}$. D. Mean harmonicity as a function of frequency. Simulations with partial-position constraint, in the presence of $\operatorname{SDN} N_{m}\left(\sigma^{\varepsilon}=2\right)$. Simulation duration was $120 \mathrm{~s}$.

\section{Fitts' law}

In the presence of noise, scaling laws (see above) define a tripartite relationship between movement amplitude, duration and endpoint variability. We explored this relationship for discrete and rhythmic (reciprocal) movements.

\section{Discrete Fitts' law}

We simulated series of movements of different amplitudes and with via-points at different positions corresponding to the scaling strategies of Figure 8B (absolute goal setting policy) and $8 \mathrm{C}$ (relative goal setting policy) in the presence of $S D N_{m}$ (see below for the influence of noise on Fitts' law; Figure 16). We measured actual movement amplitude $(A)$, duration $(M T$, to keep a traditional notation) and endpoint variability $(W)$, and we analyzed the relationship between the effective index of difficulty $I D=\log _{2}(2 A / W)$ and $M T$. As expected from Fitts' 
law, $M T$ was a linear function of $I D$ (Figure 11A,B). We note that the actual range of $I D$ is arbitrary: changing the level of noise or the definition of endpoint variability would shift the range of $I D$ with no effect on $M T$ (this remark applies to all the simulations involving a calculus of $I D)$. Time to peak velocity and time after peak velocity increased with $I D$ for absolute goal setting policy (Figure 11C) while time to peak velocity remained constant for relative goal setting policy (Figure 11D). These two strategies were observed in the study of discrete Fitts' law (Poletti, Sleimen-Malkoun, Temprado, \& Lemaire, 2015; SleimenMalkoun, Temprado, \& Berton, 2013; Temprado, Sleimen-Malkoun, Lemaire, Rey-Robert, Retornaz, \& Berton, 2013). Note that, for each amplitude, peak velocity decreased with $I D$ for the relative goal setting policy (Figure 11B, inset; MacKenzie, Marteniuk, Dugas, Liske, \& Eckmeier, 1987). Fitts' law was observed with the zero-value method, but not with the current-value method.

Figure 11 . Discrete Fitts' law. A. Movement duration as a function of $I D .5$
amplitudes $(0.1-0.5 \mathrm{~m})$, via-points at 5 equi-spaced distances $(0.11,0.132,0.155$,
$0.178,0.2 \mathrm{~m})$. Simulations with full-state constraint, zero-value method, absolute
goal setting policy. B. Movement duration as a function of $I D .5$ amplitudes $(0.1-$
$0.5 \mathrm{~m})$, via-points at 5 fractions $(0.65,0.725,0.8,0.875,0.95)$. Simulations with
full-state constraint, zero-value method, relative goal setting policy. Inset: peak
velocity as a function of $I D$. (diamond) $0.1 \mathrm{~m}$; (up triangle) $0.2 \mathrm{~m}$; (down
triangle) $0.3 \mathrm{~m}$; (left triangle) $0.4 \mathrm{~m}$; (right triangle) $0.5 \mathrm{~m}$. C. Time to peak
(black) and time after peak (gray) velocity as a function of function of $I D$ for data
in A. D. Time to peak (black) and time after peak (gray) velocity as a function of
function of $I D$ for data in B. Simulations in the presence of $S D N_{m}\left(\sigma^{\varepsilon}=1\right)$.
Variability calculated over 500 trials per condition.

\section{Rhythmical (reciprocal) Fitts' law}

We simulated series of rhythmical movements of a given amplitude as explained above (Figure 10) for frequencies in the range $0.7-2.5 \mathrm{~Hz}$. As movement amplitude decreases with frequency (Figure 10C, inset), compensatory changes in movement amplitude with frequency were used to obtain the desired amplitude (Figure 12B, inset). For the given amplitude, 
movement duration and time to peak velocity increased linearly with $I D$ for $I D$ below 4 (Figure 12A). Dwell time increased with $I D$ (Figure 12B) and harmonicity decreased for ID below 4 (Figure 12C). These observations are consistent with experimental results for low IDs (Boyle \& Shea, 2013; Buchanan, 2013). The behavior changed above $I D=4$ (low frequencies) consistent with a transition between rhythmic and discrete movements (Huys, Fernandez, Bootsma, \& Jirsa, 2010). The data for $I D>4$ are shown, but are wrong in the sense that a different mechanism is involved at higher IDs (lower frequencies; see above, Figure 11B,D).

The rhythmical movements obeyed to Fitts' law, i.e. a univocal relationship was found between $I D$ and movement duration for different amplitudes (Figure 13A). Peak velocity decreased with $I D$ as observed experimentally (Figure 13B; Boyle \& Shea, 2013). A rhythmical Fitts' law was not observed for partial-position/velocity and full-state constraints.

Figure 12. Rhythmical Fitts' law (single amplitude). A. Movement duration (circle) and time to peak velocity (square) as a function of ID. Amplitude was $0.2 \mathrm{~m}$. B. Dwell time. Inset: compensatory changes in amplitude as a function of frequency. Dotted line corresponds to $0.2 \mathrm{~m}$ (desired amplitude). C. Harmonicity. (gray lines) data from Buchanan (2013). 15 frequencies in the range 0.7-2.5 Hz. Simulations with partial-position constraint, in the presence of $\operatorname{SDN}_{m}\left(\sigma^{\varepsilon}=2\right)$. Variability calculated over 500 trials per condition.

Figure 13. Rhythmical Fitts' law (multiple amplitudes). A. Movement duration as a function of $I D$ for 3 amplitudes: (red) $0.1 \mathrm{~m}$; (black) $0.2 \mathrm{~m}$; (green) $0.3 \mathrm{~m}$. B. Peak velocity as a function of $I D$. Same parameters as in Figure 12.

\section{Timing}

Movements can be made toward spatial targets, but also toward temporal events (e.g. the beat of a metronome; Howarth, Beggs, \& Bowden, 1971). There is evidence that movements directed at spatial and temporal goals are subserved by different processes (Howarth et al., 
1971; Huys et al., 2010). For the model, this means that the task representation used to produce rhythmic movements (see above) is not appropriate to explain how movements get synchronized with a metronome. An attempt to address this issue is the following. We observe that the kinematics of a movement toward a temporal goal have peculiar features, i.e. its velocity profile is asymmetrical and peak velocity occurs late in the movement (Cos, Girard, \& Guigon, 2015; Craig, Pepping, \& Grealy, 2005; Port, Lee, Dassonville, \& Georgopoulos, 1997; Rieger, 2007; Walter \& Rieger, 2012). Furthermore time to peak velocity increases all the more so that the period of the metronome increases (Cos et al., 2015). We assume that this property is specific to timing as it has never been reported in other motor conditions. We searched for a task representation of timing in terms of viapoints. The size of peak velocities depends on the distance between via-points, thus to obtain larger velocity peaks later in the movement, it is sufficient to consider nearby via-points at the begin of the movement and more distant via-points later. Accordingly, the distance between the via-points $n-1$ and $n$ was chosen to be $n f A$ where $A$ is the movement amplitude and $f$ a parameter in $[0,1]$. Simulated velocity profiles are shown in Figure 14A. Time to peak velocity increased with movement duration and occurred later in proportion of duration (Figure 14B). Variability in movement duration increased with movement duration in the presence of noise on the goal time (Figure 14A, inset). $S D N_{m}$ was not sufficient to obtain this kind of variability.

Figure 14. Simulation of movements with late peak velocity. A. Velocity profiles.
See Text for explanation. Colors from blue to red:
$f=0.025,0.05,0.075,0.1,0.125,0.15,0.2,0.3$. Movement amplitude was $0.2 \mathrm{~m}$.
Inset: standard deviation of movement duration as a function of movement
duration. B. Relationship between movement duration and time to peak velocity
in s (left axis, open squares) and $\%$ of movement duration (right axis, closed
squares). Simulations with full-state constraint, zero-value method, relative goal
setting policy, in the presence of $S D N_{m}\left(\sigma^{\varepsilon}=2\right)$ and noise on the goal time
$\left(\sigma^{\gamma}=0.005\right)$. Variability calculated over 500 trials per condition.


We assessed the relationship between amplitude, duration and variability for timing movements as we did for the study of Fitts' law. Movement duration increased with the index of difficulty, but the slope was steeper than for a Fitts' task (Figure 15A; Howarth et al., 1971; Huys et al., 2010). Spatial variability increased linearly with average velocity (Figure 15B; Schmidt, Zelaznik, Hawkins, Franck, \& Quinn, 1979; Wright \& Meyer, 1983). Temporal variability increased linearly with movement duration (Figure 15C; Bongers, Fernandez, \& Bootsma, 2009). These results show that the model can account for specific characteristics of speed/accuracy tradeoff in a timing task. We note that the model is unable to account for the presence of pauses that precede movements toward temporal events in synchronization tasks (Cos et al., 2015; Donnet, Bartolo, Fernandes, Cunha, Prado, \& Merchant, 2014; Hove \& Keller, 2010).

The robustness of these results is the following. The results remained qualitatively similar under the current-value method, for partial position-velocity and partial-position constraint, in the absence of $S D N_{m}$. The structure of temporal variability (Figure 15C) disappeared in the absence of temporal noise. The results were lost with absolute goal setting, in the presence of $S D N_{S}$ and with noise only on the receding horizon.

Figure 15. Tripartite relationship between amplitude, duration and variability for timing movements. A. Movement duration as a function $I D$. Three movement amplitudes $(0.2,0.3,0.4 \mathrm{~m})$ and 10 fractions $(f$ between 0.02 and 0.2$)$. Gray lines from Howarth et al. (1971), fig. 3: (dashed) data from Fitts (1954); (solid) timing task. B. Spatial variability as a function of average velocity. C. Temporal variability as a function of movement duration. Simulations with full-state constraint, zero-value method, relative goal setting policy, in the presence of $\operatorname{SDN}_{m}\left(\sigma^{\varepsilon}=2\right)$ and noise on the goal time $\left(\sigma^{\gamma}=0.005\right)$. Variability calculated over 500 trials per condition (amplitude, fraction).

\section{Structure of variability and the nature of noise}

In the preceding simulations, we used sources of white Gaussian noise to explain variability.

We address here the role of the different sources. 
We have shown that discrete Fitts' law can be explained by the presence of $S D N_{m}$ (Figure 11). We assessed the basic tripartite relationship between amplitude, duration and variability for the different types of noise (except $\operatorname{SIN}_{m}$ which has a deleterious effect on control). The simulations confirmed the proper role of $S D N_{m}$ (Figure 16, black; see Figure 11A) and showed that variability produced by the other sources of noise did not comply to Fitts' law, i.e. there was no univocal relationship between movement duration and ID (Figure 16, red, green, blue, purple, yellow).

Figure 16. Simulation of discrete Fitts' law for different sources of noise. (black)
$S D N_{m}\left(\sigma^{\varepsilon}=2\right) ;($ red $) S I N_{S}\left(\sigma^{\omega}=0.0001\right)$; (green) $S D N_{S}\left(\sigma^{\epsilon}=0.5\right) ;($ blue $) T_{H}$
$\left(\sigma^{\zeta}=0.2\right)$; (purple) $T_{G}\left(\sigma^{\gamma}=0.2\right)$; (yellow) $x^{G}\left(\sigma^{\chi}=0.01\right)$. Noise standard
deviations were chosen to obtain similar ranges of $I D$, except for noise on $T_{G}$
which cannot produce a larger variability. Only the type of noise indicated was
present. 3 amplitudes $(0.2,0.25,0.3 \mathrm{~m})$. Variability calculated over 500 trials per
condition.

When participants repeatedly produce movements of a given amplitude, movement duration $(M T)$ covaries with peak velocity $(P V)$, i.e. $P V \propto M T^{-q}$ with $q \approx 0.8$ (van Beers, Haggard, \& Wolpert, 2004; see also Messier \& Kalaska, 1999). This property was found in the model for $S D N_{m}$, noise on $T_{H}$ and noise on $T_{G}$ (Figures $17 \mathrm{~A}, \mathrm{~B}, \mathrm{C}$ ), although the velocity profiles were peculiar for the two latter noises (Figure 17A, inset; 17B).

\footnotetext{
Figure 17. Variability of velocity profiles. A. Velocity profiles (gray lines) and peak velocity (black circle) for $S D N_{m}\left(\sigma^{\varepsilon}=2\right)$. 100 trials are shown. Inset: same for $T_{H}$ (blue) and $T_{G}$ (purple) noise. B. Relationship between peak velocity and movement duration for the data in A. 500 trials are shown. C. Exponent $q$ of the relationship between peak velocity and movement duration vs $R^{2}$ of the relationship. Vertical dashed line indicates $q=0.8$ (expected value; van Beers et al., 2004). (black) $S D N_{m}\left(\sigma^{\varepsilon}=2\right)$; (red) $\operatorname{SIN}_{s}\left(\sigma^{\omega}=0.00005\right)$; (green) $S D N_{s}$ $\left(\sigma^{\epsilon}=0.25\right)$; (blue) $T_{H}\left(\sigma^{\zeta}=0.18\right)$; (purple) $T_{G}\left(\sigma^{\gamma}=0.2\right)$; (yellow) $\boldsymbol{x}^{G}$ $\left(\sigma^{\chi}=0.004\right)$. Standard deviations of noise were chosen to obtain an $I D \approx 7$, except for $T_{G}$. Simulations with full-state constraint, zero-value method, absolute goal setting policy. Amplitude $0.1 \mathrm{~m}$, via-point at $0.07 \mathrm{~m}$, chosen to obtain a movement duration around $0.4 \mathrm{~s}$ as in van Beers et al. (2004). Variability calculated over 500 trials.
} 
We assessed basic aspects of correlation, spatial and temporal variability across repetitions of a movement of a given amplitude (Figure 18): 1. correlation between position at kinematic landmarks and endpoint position (Heath, Westwood, \& Binsted, 2004; Figure 18A); 2. correlation between position at normalized time and endpoint position (Heath, Neely, \& Krigolson, 2008; Figure 18B); 3. correlation between the value of kinematic landmarks (Messier \& Kalaska, 1999; Figure 18C); 4. positional standard deviation at normalized time (Liu \& Todorov, 2007; Figure 18D); 5. positional standard deviation of kinematic landmarks (Khan, Elliot, Coull, Chua, \& Lyons, 2002; Figure 18E); 6. temporal standard deviation of kinematic landmarks (Valdez \& Amazeen, 2008; Figure 18F). Each panel in Figure 18 shows simulations with 6 types of noise (colored lines, circles) and experimental data (gray lines, triangles) which are characteristic of properties reported in the literature. We observed again that the presence of $S D N_{m}$ (black lines compared to gray lines) provides a good quantitative account of the structure of variability of point-to-point movements. The presence of the other sources of noise led to contrasted results in particular as far as correlations were concerned (Figure 18A,B,C). Interestingly, both execution and planning noises can account for classical observations on the time course of positional variance (Figure 18D; Krüger, Eggert, \& Straube, 2011; Krüger, Straube, \& Eggert, 2017; Liu \& Todorov, 2007; Mosier, Scheidt, Acosta, \& Mussa-Ivaldi, 2005; Osu, Morishige, Nakanishi, Miyamoto, \& Kawato, 2015; Selen, Beek, \& van Dieën, 2006) which is generally thought to arise from execution noise (Guigon et al., 2008b; Todorov \& Jordan, 2002).

Figure 18. Variability of kinematic landmarks. A. Correlation coefficient between position at peak acceleration (PA), peak velocity (PV) and peak deceleration (PD) and endpoint position. The green curve is superimposed on the red one. Data from Heath et al. (2004), fig. 7. B. Correlation coefficient between spatial position at normalized times and endpoint position. Data from Heath et al. (2008), fig. 7. C. Mean correlation coefficient between kinematic markers (A, amplitude). Data from Messier and Kalaska (1999), fig. 11. D. Positional standard deviation at normalized times. Data from Liu and Todorov (2007), 
fig. 1e. E. Positional standard deviation at kinematic markers in percent of movement amplitude. Data from Khan et al. (2002), fig. 5. F. Temporal standard deviation at kinematic markers in percent of movement amplitude. Data from Valdez and Amazeen (2008), fig. 8. (gray, triangle) experimental data; (black) $S D N_{m}\left(\sigma^{\varepsilon}=2\right)$; (red) $S I N_{s}\left(\sigma^{\omega}=0.000075\right) ;$ (green) $S D N_{S}\left(\sigma^{\epsilon}=0.4\right) ;($ blue $)$ $T_{H}\left(\sigma^{\zeta}=0.25\right)$; (purple) $T_{G}\left(\sigma^{\gamma}=0.2\right)$; (yellow) $x^{G}\left(\sigma^{\chi}=0.01\right)$. Standard deviations of noise were chosen to obtain similar ranges of positional standard deviation (D), except for $T_{G}$. Simulations with full-state constraint, zero-value method, absolute goal setting. Amplitude $0.2 \mathrm{~m}$, via-point at $0.18 \mathrm{~m}$. Variability calculated over 500 trials.

\section{Discussion}

We presented an ensemble of simulations of motor behaviors designed following two guidelines. First they all involved the same control process. Second they each contained a specific description of a task (task representation) in terms of a spatiotemporal sequence of goals. The sequence was in general naturally related to the task, sometimes not so naturally but intuitively related to the task (movements of intermediate duration), and in a case the construction was more speculative (timing). If all the results may not be equally convincing, the overall impression is that the proposed theory has a large explanatory power that extends across many aspects of motor control. Several aspects of this work (submovement, intermittency) have been comprehensively discussed previously (Guigon et al., 2019) and are not addressed here.

\section{Disclaimer}

The present work should not be considered as a subservient extension of a dominant theory (computational theory of motor control; Scott, 2004; Todorov \& Jordan, 2002; Todorov, 2004; Wolpert \& Ghahramani, 2000), but as the outcome of a slow and in-depth progression in the field of motor control (Guigon, 2010, 2011; Guigon et al., 2019; Rigoux \& Guigon, 2012). It acknowledges past and present debates on motor control (Ajemian \& Hogan, 2010; Feldman \& Levin, 1995; Friston, 2011; Mohan, Bhat, \& Morasso, 2019; Turvey, 1977), 
recognizes the existence of divergent positions, and makes some attempts toward a reconciliation. Using a receding horizon renders the control process stationary (i.e. independent of time) and intrinsically flexible in agreement with the dynamical systems approach to motor control (Kelso, 1995). The receding horizon also provides a natural continuity between movement and posture (Guigon, 2010) as in the equilibrium-point theory (Feldman \& Levin, 1995). Yet the proposed theory remains sensitive to numerous criticisms attached to the representational view of action (Warren, 2006). Instead of trying to argue in vain against these criticisms, we developed two points based on the position/force dichotomy and the structured nature of motor variability that illustrate the power of the computational approach over other modeling frameworks (see below Debates on motor control). It is not a manoeuvre to bypass a debate, but a way to focus on technical arguments that are amenable to experimental testing rather than conceptual and philosophical arguments.

\section{Time in motor control}

A central difficulty of the computational approach to motor control is how time is processed during the course of an action. By construction, no movement is possible without choosing in advance a time horizon (Equation 1). If no other principle is available, the time horizon remains fixed along the course of the movement, leading to a nonstationary control policy and an absence of flexibility in time. A paradoxical aspect of the proposed theory is to address these difficulties by setting two rigidly defined time constants (receding horizon, update period of goals). Control with a receding horizon benefits from an automatic updating of the time to achieve goals, which is necessary when perturbations are encountered (Liu \& Todorov, 2007; Pélisson, Prablanc, Goodale, \& Jeannerod, 1986; Shadmehr \& Mussa-Ivaldi, 1994). Although the initial time horizon is set to the receding horizon, actual movement duration is an emergent property of the interaction between the controller and the 
environment. Movement duration also depends on self-selected goals (via-points) updated at a fixed period. Simple rules for the choice of via-points produce realistic scaling laws, speed/accuracy tradeoffs, and synchronization movements. A time series of spatial goals is an appropriate proxy for the specification of movement duration, precision and timing. The theory provides a principled approach to time processing in motor control which is consistent with the existence of isochronous behaviors and the nonsmooth aspect of most movements expect the fastest ones.

\section{Isochrony}

A core property of the model is the production of isochronous actions. The origin of isochrony is unknown, but is considered here as a native characteristic of the model that underlies all other emergent properties. This view is consistent with the idea that time is given priority over space in motor control (Ashton, 1976; Lashley, 1951). For instance, in speech production, consistent timing is essential for intelligibility of speech (Faulkner \& Rosen, 1999) and is preserved in patients with Parkinson's disease at the expense of amplitudes and velocities of lip displacements (Caligiuri, 1987; Connor, Abbs, Cole, \& Gracco, 1989; Walsh \& Smith, 2012). In the production of rhythmic arm movements, LevyTzedek, Ben Tov, \& Karniel (2011) have shown that the participants exerted a stronger control on movement frequency than on amplitude or velocity.

Isochrony is not a priori a unitary concept. It has been observed for fast, discrete movements (Bryan, 1892; Jeannerod, 1984) and for slower, continuous motor activities (drawing, handwriting; Denier van der Gon \& Thuring, 1965; Lacquaniti, Terzuolo, \& Viviani, 1983; Viviani \& McCollum, 1983). In the former case, isochrony could be a direct consequence of control with a receding horizon. In the latter, it could be due to both the receding horizon and the representation of task by series of goals updated at a fixed 
frequency, i.e. isochronous actions are those that have the same number of via-points irrespective of their amplitude (Figure 5). The failure to keep being perfectly isochronous in drawing circle of increasing size (Bennequin, Fuchs, Berthoz, \& Flash, 2009; Viviani \& Cenzato, 1985; Viviani \& Schneider, 1991) could be explained by a strategic increase in the number of via-points with the perimeter of the circle to avoid an excessive increase in energetic expenditure, peak velocity, or variability (speed/accuracy tradeoff). Interestingly, the principles that lead to isochrony also account for the existence of power laws (Figure 6; Huh \& Sejnowski, 2015; Lacquaniti et al., 1983; Zago et al., 2018). Accordingly, the framework proposed by Bennequin et al. (2009) to explain isochrony and power laws in terms of multiple geometries might be unwarrantedly complex.

An open question is whether an optimality principle could account for isochrony. As noted previously, a cost of time (Harris \& Wolpert, 2006; Hoff, 1994; Shadmehr et al., 2010) could explain the compensatory regulation of movement speed with amplitude to maintain movement duration approximately constant. Yet we would never obtain a strict isochrony since it is not a rational behavior to spend more cost to keep a constant duration. A possible normative account of isochrony can be derived in the framework of reward/effort-based optimal motor control (Rigoux \& Guigon, 2012). We assume that the utility of producing an action of intensity $I$ (e.g. force, amplitude) and duration $T$ to obtain a reward $\rho$ is $J=\rho / T-$ $\varepsilon I^{2} / T^{2}$ (which is a simplified version of the model developed in Rigoux \& Guigon, 2012; $\varepsilon$ is a conversion factor) which defines a trade-off between the benefits and costs of an action. Maximum utility (with respect to $T$ ) is $J^{*}=\rho^{2} / 4 \varepsilon I^{2}$ and is obtained for $T^{*}=2 \varepsilon I^{2} / \rho$. We also assume that the reward $\rho$ can be interpreted as a "motivational investment", i.e. how hard we are ready to work for a given action, and we search for a function $\rho=\rho(I)$ which maximizes 


$$
\int \frac{J^{*}(I)}{\rho(I)} d I \text { (Equation 15) }
$$

(ratio between utility and investment across the range of action intensities). Using the calculus of variations, the function $\rho$ should obey to the differential equation

$$
I^{2} \dot{\rho}(I)-2 I \rho(I)=0,
$$

which gives $\rho(I)=K I^{2}$ where $K$ is a constant. The optimal utility is $J^{*}=K \rho / 4$ and the optimal time is $T^{*}=2 / K$ which is constant. In this framework, isochrony is the outcome of an optimization process (Equation 15). Although it is interesting to have a normative explanation of isochrony, it is unclear whether this explanation is superior and more informative than a basic explanation based on the role of isochrony in the synchronization of multiple neural and behavioral events.

\section{Handwriting}

Morasso and Mussa-Ivaldi (1982) distinguished muscle-oriented and space-oriented models of trajectory formation. In the former models, geometrical and mechanical (elastic) properties of muscles create parametric oscillatory dynamics that produce curved trajectories, e.g. drawings, letters (Hollerbach, 1981; Singer \& Tishby, 1994). These models foreshadowed refined developments in the framework of the dynamical approach to motor control in which interactions on multiple time scales and between multiple dynamical patterns produce sequential, coordinated actions (e.g. handwriting; Huys et al., 2014; Perdikis, Huys, \& Jirsa, 2011; for a different, but related work, see also Friston, Mattout, \& Kilner, 2011). The latter space-oriented models are based on trajectory control in space either through combinations of motor primitives (strokes; Bullock, Grossberg, \& Mannes, 1993; Edelman \& Flash, 1987; Morasso \& Mussa-Ivaldi, 1982) or through via-point representations (Gilet, Diard, \& Bessière, 2011; Meulenbroek, Rosenbaum, Thomassen, Loukopoulos, \& Vaughan, 1996; 
Wada \& Kawato, 1995; the present model). Most of these models produce realistic writing patterns, and many characteristic properties of handwriting such as isochrony and curvature/velocity relationship easily emerge. A more challenging issue is the absolute time scale of writing processes, e.g. the duration of individual strokes. Although it is difficult to pinpoint a single number (due to the heterogeneity of acquisition and data processing methods, in particular the filtering cutoff frequency), a realistic range is $0.1-0.3 \mathrm{~s}$ (Teulings \& Stelmach, 1991). The value obtained here is $\sim 0.19 \mathrm{~s}$ (16 velocity peaks in $3 \mathrm{~s}$; Figure $6 \mathrm{C}$ ) and is constant by construction. None of the models discussed above (nor other models) can account for the existence of a fixed duration of strokes because the processing time scale is in general determined by some free parameters (coefficients of a dynamics, stiffness, ...). The strength of the present theory is to enable the coexistence of processing at a constant time scale on the one hand and full temporal flexibility on the other hand.

\section{Fitts' law}

The fact that the model accounts for Fitts' law is not a surprise. The tripartite relationship between amplitude, duration and variability appears to be a ubiquitous consequence of control in the presence of multiplicative motor noise (Guigon et al., 2008a; Harris \& Wolpert, 1998; Hoff \& Arbib, 1992; Meyer, Abrams, Kornblum, Wright, \& Smith, 1988; Qian et al., 2013; Rigoux \& Guigon, 2012; Tanaka, Krakauer, \& Qian, 2006). The main contribution of the theory is to explain 3 types of Fitts' law (discrete, rhythmic, temporal) and the kinematics of movements that obey to these laws. This corresponds to a major improvement in the computational description of Fitts' law since previous models accounted for a single type of Fitts' law and only in terms of unrealistic smooth movements of arbitrary duration (Guigon et al., 2008a; Harris \& Wolpert, 1998; Jean \& Berret, 2017). 


\section{Motor variability}

Variability is a central theme in the study of motor control that goes beyond Fitts' law. Common observations are that no two movements toward the same goal are exactly the same and variability calculated along repeated trajectories is not uniform (structured variability; Darling et al., 1988; Todorov \& Jordan, 2002). Although most motor control theorists often cite Bernstein and agree with his claim that "the global structure of the movement remains the same, while the (details of the) movements never exactly repeat themselves" (citation from Meijer \& Bruijn, 2007; original article of Bernstein in Feldman \& Meijer, 1999), observations on motor variability and its structure have raised little interest and contributed little to theoretical constructs except in the computational framework (for an exception see Martin, Reimann, \& Schöner, 2019). It could be revealing to see how variability emerges in other frameworks.

Admittedly, the methodology used for the study of variability has obvious limitations. First, noise is modeled as a white Gaussian stochastic process, although other types of noise exist. Second, part of the motor variability may not be uniquely related to the presence of noise (e.g. existence of trial-to-trial corrective processes; van Beers, Brenner, \& Smeets, 2013) or to the presence of uncorrelated noise (e.g. existence of long-range correlations in series of motor actions; Slifkin \& Eder, 2012). Yet, despite these limitations, consistent conclusions can be drawn. First, signal-dependent (multiplicative) motor noise is a major determinant of variability (Figures 16, 17, 18) consistent with previous experimental and theoretical results (Guigon et al., 2008b; Harris \& Wolpert, 1998; Jones, Hamilton, \& Wolpert, 2002; Meyer et al., 1988; Todorov, 2002; Todorov \& Jordan, 2002). Second, planning noise contributes to movement variability, in particular for the production of temporal variability (Churchland, Afshar, \& Shenoy, 2006; van Beers et al., 2004), and 
possibly for the emergence of speed/accuracy tradeoff (Al Borno, Vyas, Shenoy, \& Delp, 2020).

\section{Discrete and rhythmic movements}

There is strong evidence that discrete and rhythmic movements are not of the same nature, i.e. neither a rhythmic movement results from the concatenation of discrete segments nor a discrete movement can be identified to a truncated rhythmic movement (Guiard, 1993; Hogan \& Sternad, 2007; van Mourik \& Beek, 2004). Theoretical considerations on this distinction based on motor primitives (e.g. central pattern generators) have been reviewed by Degallier and Ijspeert (2010). The central theme was related to nonlinear dynamical equations whose solution corresponds to discrete or rhythmic patterns depending on parameters (Degallier, Righetti, Natale, Nori, Metta, \& Ijspeert, 2008; de Rugy \& Sternad, 2003; Huys, Studenka, Rheaume, Zelaznik, \& Jirsa, 2008; Jirsa \& Kelso, 2005; Ronsse, Sternad, \& Lefèvre, 2009; Schaal, Kotosaka, \& Sternad, 2000; Schöner, 1990). None of the described models can account for the changes in characteristics of rhythmic movements with frequency and the transition to discrete movements at lower frequencies (Guiard, 1993; Sternad et al., 2013).

An alternative approach was described by Biess, Nagurka, \& Flash (2006) who defined discrete and rhythmic movements as solutions to optimal control problems with different boundary conditions. Our view of the difference between discrete and rhythmic movements is consistent with this idea. In this framework, rhythmicity is not the consequence of a continuous, autonomous dynamics, but of a discrete, periodic guidance. It should be noted that the transition between rhythmic and discrete movements observed around $1 \mathrm{~Hz}$ in the model does not correspond to a qualitative change in behavior (e.g. a bifurcation). A velocity threshold was used to detect "artificial" dwell periods, but in fact, the underlying velocity pattern changed with frequency along a continuum. 
According to the theory, the computational specificity of rhythmic movements is related to the management of constraints, i.e. goals are defined only by positional constraints (Equation 9). Discrete movements require a broader set of constraints for the extra demands of termination, and accordingly, larger computational resources. This view is consistent with the observation that the neural substrate of rhythmic movements is a subset of the neural substrate of discrete movements (Schaal, Sternad, Osu, \& Kawato, 2004).

\section{What makes the theory work?}

The first principle (optimal feedback control) is responsible for the basic machinery of motor control (Todorov \& Jordan, 2002) and the present model adds nothing specific to this point. The second principle (receding horizon) imposes a strong constraint on movement production: movements comply with a universal relationship between duration and jerkiness (Salmond et al., 2017; Shmuelof et al., 2012). Avoiding the production of unrealistic movements (e.g. smooth long-duration movements) probably contributes to the performance of the theory. The third principle is more enigmatic. It was adopted to explain velocity fluctuations during slow movements (Guigon et al., 2019) and seems effective to specify the kinematics and duration of a large class of movements. In fact, updating of goals at $\sim 8 \mathrm{~Hz}$ is equally efficient to produce point-to-point movements of different durations and arbitrary drawing movements, and account for their properties (scaling law, speed/accuracy tradeoffs, power laws). The reason for this is unclear but it is satisfying to note that no additional hypothesis was needed to extend the model beyond the scope of slow movements.

\section{Relation to previous models}

The current theory is based on two concepts (intermittency, receding horizon) which are used in the modeling framework developed by Bye and Neilson $(2008,2010)$. In their formalism, intermittency corresponds to successive 100-ms refractory periods. During a refractory 
period, processing of incoming information is delayed to the next period and a trajectory between predicted initial state and predicted target state is planned over a variable horizon. At the end of the refractory period, the planned trajectory is executed and a new refractory period starts in parallel. Accordingly, an action consists in a concatenated sequence of fixed duration submovements. Depending on the task, the variable horizon is either a receding horizon or a fixed horizon. For instance, a fixed horizon is used in tasks calling for temporal and spatial accuracy. On the one hand, our theory agrees with the view of Bye and Neilson that intermittent processes at a $100-\mathrm{ms}$ time scale (close to $T_{G}$ ) are fundamental in motor control. For Bye and Neilson, the rationale for intermittency is to account for the welldocumented psychological refractory period (Pashler, 1994). We could endorse this view since we have no independent reason for intermittency except that it helps accounting for the characteristics of kinematic fluctuations (Guigon et al., 2019). On the other hand, there is a clear difference between the variable horizon of Bye and Neilson which can be either a fixed or a receding horizon, is task-dependent, and varies as a parameter, and our receding horizon which is task-independent and is not a varying parameter. Bye and Neilson (2008) have shown that logarithmic and linear speed-accuracy tradeoffs rely on receding and fixed horizon control, respectively. In contrast, all speed-accuracy tradeoffs and their associated kinematics emerge from receding horizon control in our modeling framework.

\section{Limitations of the theory}

A limitation of the theory is the versatility in task representation (series of goals, nature of boundary conditions) afforded by the third computational principle. A series of goals could be chosen by hand to obtain any desired motor behavior. Playing with all available states (and not solely position and velocity) would still increase the size of the space of representations. Thus, although the model has no free parameters, an open question is 
whether extended variations in implementation details and in the nature of noise are necessary to guarantee its explanatory power. A summary of all the simulations is given in Table 2. Several patterns appear. First, the full-state constraint applies to discrete point-topoint movements (gray lines). For these movements, partial constraints on the position or velocity of via-points lead to poorer results. The value method plays no specific role except for discrete Fitts' law. We could remove this notion and consider only the zero-value method. Second, continuous and rhythmic movements (white lines) involve the partial-position constraint and poorer results were obtained with other types of constraint. Third, the goal setting policy is a relevant dimension for discrete movements. Fourth, signal-dependent motor noise is central to movement variability although temporal noise is also required to explain temporal variability.

Another limitation is related to the (im)possibility to falsify the theory. We have presented all what the theory can explain, but what about aspects that cannot be explained? We have not found any property that we have tried to reproduce and for which we have obtained a wrong prediction. Yet as neither a confirmation bias nor bad faith can be definitely excluded, we need to be careful on this issue.

The scope of the theory is not known precisely. Although the theory was developed exclusively in relation to movements of the upper limbs, there is no reason why it would not apply to other bodily movements (leg, trunk, head, face, ...). Yet it is unclear how a task like locomotion can be cast in the proposed framework. It is also unclear whether it could apply to eye movements. Saccades share a number of properties with limb movements (kinematics, scaling laws, Fitts' law) and are successfully modeled in the framework of optimal control (Harris \& Wolpert, 1998, 2006; Saeb, Weber, \& Triesch, 2011; van Beers, 2008). However, 
there is a striking absence of isochrony in saccade production ${ }^{13}$ which appears incompatible with the principles of the theory.

By construction, the theory leaves no room for a role of stiffness in the production of movements. As in many other computational models, the role of stiffness is thought to be restricted to a contribution to the compensation of external perturbations. Yet this view does not acknowledge the complex and refined contribution of stiffness to motor control (Burdet, Osu, Franklin, Milner, \& Kawato, 2001). Recent models have attempted to give a more precise account of the role of stiffness in the framework of optimal control models (Berret \& Jean, 2020; Mitrovic, Klanke, Osu, Kawato, \& Vijayakumar, 2010).

\section{Debates on motor control}

The debate about motor control has a large spectrum, but has in fact been centered on two Shakespearian dilemma: To control or not to control? To model or not to model? At one end of the spectrum, an ensemble of theoretical constructs (named below DA, dynamical approach) based on the ecological approach of Gibson $(1966,1986)$, physical theories of self-organized pattern formation (Haken, 1983) and dynamical system theory (Strogatz, 1994) claim that neither a centralized control nor a centralized representation are necessary for motor coordination, and behavioral motor patterns emerge from abstract, phenomenological nonlinear dynamics corresponding to interactions between the brain, the body and the environment (Kelso, 1995; Warren, 2006). At the other end, the computational approach views the nervous system (or part of it) as a computing device that builds models of the body and the environment, and controls changes in body states to achieve goals in the environment (Kawato, 1999; Todorov \& Jordan, 2002). In between, the Passive Motion

\footnotetext{
${ }^{13}$ Yet, other eye movements such as eyelid movements (Evinger, Shaw, Peck, Manning, \& Baker, 1984) and saccades without gaze-evoked blinks (Powers, Basso, \& Evinger, 2013) are isochronous.
} 
Paradigm $^{14}$ (PMP; Mohan \& Morasso, 2011) combines classical feedback control and forward modeling, the Equilibrium Point Theory (EPT; Feldman \& Levin, 1995) is closely related to classical feedback control theory ${ }^{15}$, but denies the existence of internal models, and the Active Inference theory (AIT) exploits the notion of internal (generative) model, but views motor control as an inference problem rather than a control problem (Friston, 2011). Available discussions have in general been restricted to these dilemma, and have failed to provide decisive, convincing arguments for or against a particular theory.

Interestingly, a line of reasoning based on the distinction between position and force control (Ostry \& Feldman, 2003) lead to sharper and less consensual arguments. On the one hand, models of movement control based on the direct specification of forces fail to properly take into account the existence of posture-stabilizing mechanisms (von Holst \& Mittelstaedt, 1950/1973), and incorrectly predict patterns of muscular coactivation for postural maintenance following a displacement (Ostry \& Feldman, 2003). On the other hand, production of movement based on position control is intrinsically compatible with postural control as a direct consequence of a postural resetting mechanism (Feldman \& Levin, 1995). In DA, EPT, PMP and AIT, the basic unified mechanism of movement production and postural control is determined by an autonomous dynamical system, and is based on a local fixed-point dynamics ${ }^{16}$, i.e. a restoring force ${ }^{17}$ is exerted which is a function of the distance to a (static or moving) equilibrium point with a constant or state-dependent (possibly nonlinear) gain (stiffness). This description fits with the notion of position control. According to these theories, postural maintenance should correspond to such fixed-point dynamics. Yet, there is

\footnotetext{
${ }^{14}$ Whether the PMP should be considered as a "computational" method or not is discussed in Mohan and Morasso (2011).

15 The control theoretical framework is not intrinsically incompatible with "noncomputational" approaches, e.g. some dynamical models can be cast in terms of the control theory (Sternad, 2000, pp 415-416).

${ }^{16}$ In the dynamical approach, the dynamics can also be governed by a limit-cycle attractor.

${ }^{17}$ In most cases, a second-order dynamics is used which allows to use the term "force". Yet this term is not quite appropriate for an abstract dynamics.
} 
strong evidence that posture cannot be uniquely described by this kind of process. For whole body posture, ankle stiffness is in general too low to guarantee passive stabilization, which calls for the existence of an active, predictive postural control mechanism (Amiri \& Kearney, 2020; Bottaro, Casadio, Morasso, \& Sanguineti, 2005; Casadio, Morasso, \& Sanguineti, 2005; Loram, Kelly, \& Lakie, 2001; Loram \& Lakie, 2002; Morasso \& Schieppati, 1999; Morasso \& Sanguineti, 2002). Studies on the manual stabilization of unstable objects revealed two strategies: a high-stiffness strategy and a low-stiffness, intermittent, predictive strategy (Lakie, Caplan, \& Loram, 2003; Saha \& Morasso, 2012). These observations indicate that theories based on position control would need to be amended to account for the need of predictive control, which in fact would violate their very principles. The computational approach is less sensitive to this debate between position and force control because it is versatile and can be cast in different ways, e.g. as predictive control of a positional variable (Guigon, 2010).

The ultimate goal of a theorist is, on the one hand, to design an overarching theory in his field of research and, one the other hand, to elaborate an argumentative discourse that supports his theory and can dismiss alternative, concurrent proposals. Although the two tasks are important on their own, the latter should not be used shamelessly to conceal difficulties in the former. At its inception (Kugler, Kelso, \& Turvey, 1980), the dynamical approach was conceived as a formal alternative to the cognitive/information-processing theory of action, but with no proper content. With the advent of the finger "wipers" task (Kelso, 1984; Kelso, Holt, Rubin, \& Kugler, 1981), ensuing modeling (Haken, Kelso, \& Bunz, 1985) and later developments (review in Beek, Peper, \& Stegeman, 1995; Kelso, 1995), the dynamical approach became a well-established theory of action encompassing behavioral and neural levels (Jirsa, Friedrich, Haken, \& Kelso, 1994; Schöner, 1990, 1994). Yet its explanatory power remains largely limited to variations around the original task (Sternad, 1998, 2000). 
Recent works (Huys et al., 2014; Warren, 2006) involved long arguments and long methodological developments, but produced little insights outside the historical scope of the theory. In the meantime, several critical issues remain unsolved. The first issue is related to the possibility that common principles apply to rhythmic and discrete actions (Beek et al., 1995; Daffertshofer, van Veen, Ton, \& Huys, 2014). The second issue concerns the lack of a general method to guide the design of a dynamical system for reproducing a given behavior (Ijspeert, Nakanishi, Hoffmann, Pastor, \& Schaal, 2013). A possible solution would be to use computational tools (optimization, machine learning techniques) to discover or learn appropriate dynamics (Ijspeert et al., 2013; Schaal, Mohajerian, \& Ijspeert, 2007), but it is not in the spirit of the dynamical approach. The third issue is related to the very nature of emergent trajectories defined in general in an abstract space (e.g. relative phase in Haken et al., 1985; but for a definition in task or body space, see Haken et al. 1985; Saltzman \& Kelso, 1987; Schaal et al., 2007) and how they can be translated into actual displacements of a necessarily mechanical object (e.g. a finger). The trajectories should thus be considered as an input to a trajectory-following system. At this stage, the difficulty is not solely related to the lack of flexibility of trajectory-based control (Schaal et al., 2007), but more deeply to the fact that remaining redundancies below the level of trajectory definition (e.g. in muscle or neural space) are neither considered nor exploited (Todorov \& Jordan, 2002). The fourth issue is the intrinsic temporal and spatial invariance of the solutions of autonomous dynamical systems which can be interesting in robotics (Schaal et al., 2007), but is not consistent with human motor behavior (Gentner, 1987; Smith, Goffman, Zelaznik, Ying, \& McGillem, 1995). The last two issues also apply to the Equilibrium-Point Theory (Feldman \& Levin, 1995) and the Active Inference theory (Friston, 2011; Friston et al., 2011) since they are cast in terms of a trajectory-following problem. The Passive Motion Paradigm is by construction more computational and versatile than DA, EPT and AIT, and in fact, by different aspects, not so 
far from optimal control models (Mohan \& Morasso, 2011). Yet this theory would need to be further develop toward an overarching account of motor control.

\section{Conclusion}

In keeping with the theory of Todorov and Jordan (2002), the present theory explains characteristics of motor control as by-products of computational principles (optimal feedback control, receding horizon, task representation by a series of goals). None of the principles is new (Guigon et al., 2019; Todorov \& Jordan, 2002), but their assemblage in an overarching theory of motor control is new. These principles are sufficient to provide a detailed account of a large set of motor behaviors (discrete, continuous, rhythmic and timing actions) and properties (scaling laws, power laws, speed-accuracy tradeoffs). The theory significantly extends our understanding of action production in the framework of control theory. 


\section{Tables}

\begin{tabular}{|c|l|}
\hline Time & Explanation \\
\hline$\Delta$ & duration of a simulation \\
\hline$t$ & current time during the simulation \\
\hline$\delta$ & timestep of the simulation - for numerical integration \\
\hline$T_{H}$ & receding horizon - in the model the value of $T_{H}$ is constant $(0.28 \mathrm{~s})$ \\
\hline$T_{0}$ & fixed horizon - used only to contrast with the receding horizon \\
\hline$T(t)$ & $\begin{array}{l}\text { time interval to reach a goal at time } t \text { (Equation } 1)- \text { the value of } T(t) \text { can } \\
\text { be: } T_{0} \text { (fixed horizon control); } \infty \text { (infinite horizon control); or } t+T_{H} \\
\text { (receding horizon control) }- \text { only the receding horizon control is used }\end{array}$ \\
\hline$T_{G}$ & $\begin{array}{l}\text { period at which goals are updated in the absence of temporal constraints }- \\
\text { in the model the value of } T_{G} \text { is constant }(0.13 \mathrm{~s})\end{array}$ \\
\hline$T_{\text {step }}$ & $\begin{array}{l}\text { period at which goals are updated in general (Figure } 1 \mathrm{C})- \text { the value of } \\
T_{\text {step }} \text { is: } T_{G} \text { in the absence of temporal constraints; any value in the presence } \\
\text { of temporal constraints (e.g. period of a metronome) }\end{array}$ \\
\hline$t_{k(k=1, \ldots N)}$ & times at which goals are updated (Equation 6$)-t_{k+1}-t_{k}=T_{\text {step }}$ \\
\hline
\end{tabular}

Table 1. Summary on time notations.

\begin{tabular}{|l|c|c|c|c|c|c|c|c|l|}
\hline exp & constr & var & VP & TA & VM & GS & noise & Fig & comment \\
\hline fastest & full-state & $p$ & - & $(11)$ & - & - & - & 2 & - \\
\hline slow & full-state & $p v$ & $\begin{array}{c}(12 / \\
14)\end{array}$ & - & Z/C & A & - & 3 & $\begin{array}{l}\text { Does not work with relative goal setting. } \\
\text { Works with partial pv constraints. }\end{array}$ \\
\hline interm. & full-state & $p$ & $(11)$ & $(11)$ & Z/C & A/R & - & 4 & - \\
\hline drawing & $\begin{array}{c}\text { partial } \\
\text { position }\end{array}$ & $p$ & $(9)$ & - & - & $\mathrm{A}$ & $S D N_{m}$ & 5,6 & $\begin{array}{l}\text { Poorer fit with partial pv and full-state } \\
\text { constraints, and with relative goal setting. }\end{array}$ \\
\hline writing & $\begin{array}{c}\text { partial } \\
\text { position }\end{array}$ & $p$ & $(9)$ & - & - & $\mathrm{A}$ & $S D N_{m}$ & 7 & $\begin{array}{l}\text { Poorer fit with partial pv and full-state } \\
\text { constraints, and with relative goal setting. }\end{array}$ \\
\hline $\begin{array}{l}\text { scaling } \\
\text { laws }\end{array}$ & $\begin{array}{c}\text { full-state } \\
\text { fiscte }\end{array}$ & $p$ & $(11)$ & $(11)$ & $\mathrm{Z} / \mathrm{C}$ & $\mathrm{A}$ & - & 8,9 & $\begin{array}{l}\text { Poorer scaling with partial p and pv } \\
\text { constraints. }\end{array}$ \\
\hline full-state & $p$ & $(11)$ & $(11)$ & $\mathrm{Z}$ & $\mathrm{A}$ & $S D N_{m}$ & 11 & $\begin{array}{l}\text { Loss of Fitts' law with current-value } \\
\text { method. Fundamental role of } S D N_{m} .\end{array}$ \\
\hline rhythmic & $\begin{array}{c}\text { partial } \\
\text { position }\end{array}$ & $p$ & - & $(9)$ & - & - & $S D N_{m}$ & 10 & $\begin{array}{l}\text { Qualitatively similar results with partial pv } \\
\text { and full-state constraints. }\end{array}$ \\
\hline
\end{tabular}




\begin{tabular}{|l|l|l|l|l|l|l|l|l|l|}
\hline & & & & & & & & & method. \\
\hline $\begin{array}{l}\text { rhythmic } \\
\text { Fitts }\end{array}$ & $\begin{array}{c}\text { partial } \\
\text { position }\end{array}$ & $p$ & - & $(9)$ & - & - & $S D N_{m}$ & 12,13 & $\begin{array}{l}\text { Loss of Fitts' law with partial pv and full- } \\
\text { state constraints. }\end{array}$ \\
\hline timing & full-state & $p$ & $(11)$ & $(11)$ & $\mathrm{Z} / \mathrm{C}$ & $\mathrm{R}$ & $T_{G}$ & 14,15 & $\begin{array}{l}\text { Importance of temporal noise to account } \\
\text { for temporal variability. }\end{array}$ \\
\hline
\end{tabular}

Table 2. Comparison between the simulations. constr: boundary constraint; var: variable; $p$ : position; $v$ : velocity; VP: via-point; TA: target; VM: value method; Z: zero-value; C: current-value; GS: goal setting policy; A: absolute; R: relative. Number in parentheses refer to equations. Gray lines indicate discrete movements.

\section{Appendix A - Parametric study of power laws}

A parametric analysis of the power laws obtained in drawing (Figure 6) has been performed.

It involved $T_{H}, T_{G}$, and the nature of boundary constraints. It shows that $T_{H}=0.28 \mathrm{~s}$, $T_{G}=0.13 \mathrm{~s}$, and the partial-position constraint give the best fit to observed data (Figure A1), which provides an independent cross-check for $T_{H}$ and $T_{G}$.

Figure A1. Parametric study of drawing power laws. A. Influence of $T_{H}$ for $T_{G}=0.13 \mathrm{~s}$ and partial-position constraint: (black) $T_{H}=0.28 \mathrm{~s} ;($ red $) T_{H}=$ $0.18 \mathrm{~s} ;$ (green) $T_{H}=0.38 \mathrm{~s}$; (blue) $T_{H}=0.48 \mathrm{~s}$. B. Influence of $T_{G}$ for $T_{H}=0.28 \mathrm{~s}$ and partial-position constraint: (black) $T_{G}=0.13 \mathrm{~s} ;$ (red) $T_{G}=$ $0.18 \mathrm{~s}$; (green) $T_{G}=0.23 \mathrm{~s}$. C. Influence of boundary constraints for $T_{H}=0.28 \mathrm{~s}$ and $T_{G}=0.13 \mathrm{~s}$ : (black) partial-position constraint; (red) partial-position/velocity constraint with current-value method; (green) partial-position/velocity constraint with zero-value method; (blue) full-state constraint with current-value method; (purple) full-state constraint with zero-value method.

\section{Appendix B - Choice of via-points}

Figure B1. Via-points. A. Position of the via-points for the point-to-point movements of Figure 8B. Absolute goal setting policy. B. Position of the viapoints for the point-to-point movements of Figure 8C. Relative goal setting policy. (diamond) start point; (plain square) via-point; (circle) target. 


\section{Appendix C - Phase transition in rhythmic movements}

A cardinal observation in the study of rhythmic movements is the frequency-dependent phase transition in bimanual coordination (Kelso, 1984), i.e. synchronized, asymmetric (parallel) finger displacements are suddenly replaced by symmetric displacements as synchronization frequency increases. A popular explanation of this phenomenon is provided by the HakenKelso-Bunz model in terms of phase transition in a parameterized nonlinear dynamical system (Haken et al., 1985). The predictive power of this model is undeniable and striking (Kelso, 1995), yet its special-purpose nature is a matter of concern (Ijspeert et al., 2013). Todorov and Jordan (2002) proposed a partial account of phase transition in the framework of optimal feedback control. They observed that in a one-dimensional sinusoidal tracking task with a redundant, 2-dof (telescopic) arm, the phase between the first segment of the arm and the endpoint changed abruptly with frequency (at $\sim 2.8 \mathrm{~Hz})^{18}$. The present theory could endorse this view to extend its explanatory power. Yet an alternative approach can be developed based on the notion of task representation. In the study of drawing movements, we have shown that the fastest two-dimensional drawing defined by internally updated via-points has 3 via-point and lasts $\sim 0.38$ s, i.e. $\sim 2.6 \mathrm{~Hz}$ for repetitive drawing (Figure 5E, inset). In this framework, the only mean to increase movement frequency is to make a one-dimensional drawing (2 via-points). If we consider that asymmetric and symmetric patterns abstractly correspond to $2 \mathrm{D}$ and $1 \mathrm{D}$ representations, respectively, phase transition is explained by the necessary change of dimension to increase movement frequency. A phase transition between drawing the figure "eight" and drawing the figure "zero" has been described by Buchanan, Kelso, \& Fuchs (1996) with a critical frequency between 1.2 and $1.4 \mathrm{~Hz}$. In the model, the figure "eight" can be drawn with different numbers of via-points (here from 8 to 4;

18 Experimentally measured transition frequencies vary considerably between participants (Kelso, 1984). In Scholz, Kelso, \& Schöner (1987), the range is 2-2.6 Hz. 
Figure $\mathrm{C} 1 \mathrm{~A}, \mathrm{~B}, \mathrm{D}, \mathrm{E}$ ). The minimum number of via-points is 4 (which can also be used to draw the figure "zero"; Figure C1F) corresponding to a frequency of $1.89 \mathrm{~Hz}$. The discrepancy between the experimental and theoretical transition frequencies may be related to what is considered as a proper "eight" representation. For instance, a transition could occur around $1.28 \mathrm{~Hz}$, between 8 and 6 points, if the 6 points are interpreted as a "zero" rather than as a "eight" (Figure C1A,C). Overall, this view of phase transition is consistent with the perceptual rather than motoric nature of bimanual coordination (Mechsner, Kerzel, Knoblich, \& Prinz, 2001).

Figure C1. Via-point representations for drawing the figure "eight" and the figure "zero" (left column) and actual drawing (right column). The starting point is indicated by a open square, 50 turns. The actual number of via-points may be different from the observed number of via-points since some via-points can be used several times. Simulations with partial-position constraint, absolute goal setting policy, in the presence of $S D N_{m}\left(\sigma^{\varepsilon}=1\right)$.

\section{References}

Ajemian, R., \& Hogan, N. (2010). Experimenting with theoretical motor neuroscience. Journal of Motor Behavior, 42, 333-342.

doi: https://dx.doi.org/10.1080/00222895.2010.529332

PMID: https://www.ncbi.nlm.nih.gov/pubmed/21184350

Al Borno, M., Vyas, S., Shenoy, K. V., \& Delp, S. (2020). High-fidelity musculoskeletal modeling reveals a motor planning contribution to the speed-accuracy tradeoff. Elife, 9, e57021.

doi: https://dx.doi.org/10.7554/eLife.57021

PMID: https://pubmed.ncbi.nlm.nih.gov/33325369

Amiri, P., \& Kearney, R. E. (2020). Patterns of muscle activation and modulation of ankle intrinsic stiffness in different postural operating conditions. Journal of Neurophysiology, 123, 743-754.

doi: https://dx.doi.org/10.1152/jn.00558.2019

PMID: https://www.ncbi.nlm.nih.gov/pubmed/31913747

Arber, S., \& Costa, R. M. (2018). Connecting neuronal circuits for movement. Science, 360, 1403-1404.

doi: https://dx.doi.org/10.1126/science.aat5994

PMID: https://www.ncbi.nlm.nih.gov/pubmed/29954969 
Ashton, R. (1976). Aspects of timing in child development. Child Development, 47, 622626.

doi: https://dx.doi.org/10.2307/1128176

Baraduc, P., Thobois, S., Gan, J., Broussolle, E., \& Desmurget, M. (2013). A common optimization principle for motor execution in healthy subjects and parkinsonian patients. Journal of Neuroscience, 33, 665-677. doi: https://dx.doi.org/10.1523/JNEUROSCI.1482-12.2013

PMID: https://www.ncbi.nlm.nih.gov/pubmed/23303945

Baron, S., \& Kleinman, D. L. (1969). The human as an optimal controller and information processor. IEEE Transactions on Man-Machine Systems, 10, 9-17. doi: https://dx.doi.org/10.1109/TMMS.1969.299875

Beek, P. J., Peper, C. E., \& Stegeman, D. (1995). Dynamical models of movement coordination. Human Movement Science, 14, 573-608. doi: https://dx.doi.org/10.1016/0167-9457(95)00028-5

Bennequin, D., Fuchs, R., Berthoz, A., \& Flash, T. (2009). Movement timing and invariance arise from several geometries. PLoS Computational Biology, 5, e1000426. doi: https://dx.doi.org/10.1371/journal.pcbi.1000426 PMID: https://www.ncbi.nlm.nih.gov/pubmed/19593380

Berio, D., Calinon, S., \& Fol Leymarie, F. (2017). Generating calligraphic trajectories with model predictive control. In Proceedings 43rd Conference on Graphics Interface (pp. 132-139). Edmonton, Alberta, Canada: Canadian Human-Computer Communications Society School of Computer Science.

doi: https://dx.doi.org/10.20380/GI2017.17

Bernstein, N. (1967). The co-ordination and regulation of movements. Oxford, England: Pergamon Press.

Berret, B., \& Jean, F. (2020). Stochastic optimal open-loop control as a theory of force and impedance planning via muscle co-contraction. PLoS Computational Biology, 16, e1007414.

doi: https://dx.doi.org/10.1371/journal.pcbi.1007414

PMID: https://www.ncbi.nlm.nih.gov/pubmed/32109941

Biess, A., Nagurka, M., \& Flash, T. (2006). Simulating discrete and rhythmic multi-joint human arm movements by optimization of nonlinear performance indices. Biological Cybernetics, 95, 31-53.

doi: https://dx.doi.org/10.1007/s00422-006-0067-7

PMID: https://www.ncbi.nlm.nih.gov/pubmed/16699783

Binet, A., \& Courtier, J. (1893). Sur la vitesse des mouvements graphiques. Revue Philosophique, 35, 664-671.

Bongers, R. M., Fernandez, L., \& Bootsma, R. J. (2009). Linear and logarithmic speedaccuracy trade-offs in reciprocal aiming result from task-specific parameterization of an 
invariant underlying dynamics. Journal of Experimental Psychology: Human Perception \& Performance, 35, 1443-1457.

doi: https://dx.doi.org/10.1037/a0015783

PMID: https://www.ncbi.nlm.nih.gov/pubmed/19803648

Bottaro, A., Casadio, M., Morasso, P. G., \& Sanguineti, V. (2005). Body sway during quiet standing: Is it the residual chattering of an intermittent stabilization process? Human Movement Science, 24, 588-615.

doi: https://dx.doi.org/10.1016/j.humov.2005.07.006

PMID: https://www.ncbi.nlm.nih.gov/pubmed/16143414

Boyle, J. B., \& Shea, C. H. (2013). Micro-movements of varying difficulties: Wrist and arm movements. Experimental Brain Research, 229, 61-73.

doi: https://dx.doi.org/10.1007/s00221-013-3590-5

PMID: https://www.ncbi.nlm.nih.gov/pubmed/23732949

Bryan, W. L. (1892). On the development of voluntary motor ability. American Journal of Psychology, 5, 125-204.

doi: https://dx.doi.org/10.2307/1410865

Bryson, A. E. (1999). Dynamic optimization. Englewood Cliffs, NJ, USA: Prentice-Hall. ISBN: http://www.isbnsearch.org/isbn/9780201361872

Bryson, A. E., \& Ho, Y.-C. (1975). Applied optimal control - Optimization, estimation, and control. New York, NY, USA: Hemisphere Publ Corp

ISBN: http://www.isbnsearch.org/isbn/9780891162285

Buchanan, J. J. (2013). Flexibility in the control of rapid aiming actions. Experimental Brain Research, 229, 47-60.

doi: https://dx.doi.org/10.1007/s00221-013-3589-y

PMID: https://www.ncbi.nlm.nih.gov/pubmed/23760281

Buchanan, J. J., Kelso, J. A. S., \& Fuchs, A. (1996). Coordination dynamics of trajectory formation. Biological Cybernetics, 74, 41-54.

doi: https://dx.doi.org/10.1007/s004220050217

PMID: https://www.ncbi.nlm.nih.gov/pubmed/8573652

Bullock, D., \& Grossberg, S. (1988). Neural dynamics of planned arm movements: Emergent invariance and speed-accuracy properties during trajectory formation. Psychological Review, 95, 49-90.

doi: https://dx.doi.org/10.1037/0033-295X.95.1.49

PMID: https://www.ncbi.nlm.nih.gov/pubmed/3281179

Bullock, D., Grossberg, S., \& Mannes, C. (1993). A neural network model for cursive script production. Biological Cybernetics, 70, 15-28.

doi: https://dx.doi.org/10.1007/BF00202562

Burdet, E., Osu, R., Franklin, D. W., Milner, T. E, \&, Kawato, M. (2001). The central nervous system stabilizes unstable dynamics by learning optimal impedance. Nature, $414,446-449$ 
doi: https://dx.doi.org/10.1038/35106566

PMID: https://www.ncbi.nlm.nih.gov/pubmed/11719805

Bye R. T., \& Neilson, P. D. (2008). The BUMP model of response planning: Variable horizon predictive control accounts for the speed-accuracy tradeoffs and velocity profiles of aimed movement. Human Movement Science, 27, 771-798.

doi: https://dx.doi.org/10.1016/j.humov.2008.04.003

PMID: https://www.ncbi.nlm.nih.gov/pubmed/18774616

Bye, R. T., \& Neilson, P. D. (2010). The BUMP model of response planning: Intermittent predictive control accounts for $10 \mathrm{~Hz}$ physiological tremor. Human Movement Science, 29, 713-736.

doi: https://dx.doi.org/10.1016/j.humov.2010.01.006

PMID: https://www.ncbi.nlm.nih.gov/pubmed/20674054

Caligiuri, M. P. (1987). Labial kinematics during speech in patients with parkinsonian rigidity. Brain, 110, 1033-1044.

doi: https://dx.doi.org/10.1093/brain/110.4.1033

PMID: https://www.ncbi.nlm.nih.gov/pubmed/3651793

Casadio, M., Morasso, P. G., \& Sanguineti, V. (2005). Direct measurement of ankle stiffness during quiet standing: Implications for control modelling and clinical application. Gait Posture, 21, 410-424.

doi: https://dx.doi.org/10.1016/j.gaitpost.2004.05.005

PMID: https://www.ncbi.nlm.nih.gov/pubmed/15886131

Churchland, M. M., Afshar, A., \& Shenoy, K. V. (2006). A central source of movement variability. Neuron, 52, 1085-1096.

doi: https://dx.doi.org/10.1016/j.neuron.2006.10.034

PMID: https://www.ncbi.nlm.nih.gov/pubmed/17178410

Connor, N. P., Abbs, J. H., Cole, K. J., \& Gracco, V. L. (1989). Parkinsonian deficits in serial multiarticulate movements for speech. Brain, 112, 997-1009.

doi: https://dx.doi.org/10.1093/brain/112.4.997

PMID: https://www.ncbi.nlm.nih.gov/pubmed/2775998

Cos, I., Girard, B., \& Guigon, E. (2015). Balancing out dwelling and moving: Optimal sensorimotor synchronization. Journal of Neurophysiology, 114, 146-158.

doi: https://dx.doi.org/10.1152/jn.00175.2015

PMID: https://www.ncbi.nlm.nih.gov/pubmed/25878154

Craig, C., Pepping, G. J., \& Grealy, M. (2005). Intercepting beats in predesignated target zones. Experimental Brain Research, 165, 490-504.

doi: https://dx.doi.org/10.1007/s00221-005-2322-x

PMID: https://www.ncbi.nlm.nih.gov/pubmed/15912367

Daffertshofer, A., van Veen, B., Ton, R., \& Huys, R. (2014). Discrete and rhythmic movements - Just a bifurcation apart? In Proceeding IEEE International Conference on Systems, Man and Cybernetics (pp. 778-783). San Diego, CA, USA: IEEE.

doi: https://dx.doi.org/10.1109/SMC.2014.6974005 
Darling, W. G., Cole, K. J., \& Abbs, J. H. (1988). Kinematic variability of grasp movements as a function of practice and movement speed. Experimental Brain Research, 73, 225-235.

doi: https://dx.doi.org/10.1007/BF00248215

PMID: https://www.ncbi.nlm.nih.gov/pubmed/3215301

Degallier, S., \& Ijspeert, A. (2010). Modeling discrete and rhythmic movements through motor primitives: A review. Biological Cybernetics, 103, 319-338.

doi: https://dx.doi.org/10.1007/s00422-010-0403-9

PMID: https://www.ncbi.nlm.nih.gov/pubmed/20697734

Degallier, S., Righetti, L., Natale, L., Nori, F., Metta, G., \& Ijspeert, A. J. (2008). A modular bio-inspired architecture for movement generation for the infant-like robot iCub. In Proceedings of the 2nd IEEE RAS/EMBS International Conference on Biomedical Robotics and Biomechatronics (pp. 795-800). Scottsdale, AZ, USA: IEEE Press.

doi: https://dx.doi.org/10.1109/BIOROB.2008.4762847

Denier van der Gon, J. J., \& Thuring, J. P. (1965). The guiding of human writing movements. Kybernetik, 2, 145-148.

doi: https://dx.doi.org/10.1007/BF00272310

de Rugy A., \& Sternad, D. (2003). Interaction between discrete and rhythmic movements: Reaction time and phase of discrete movement initiation against oscillatory movements. Brain Research, 994, 160-174.

doi: https://dx.doi.org/10.1016/j.brainres.2003.09.031

PMID: https://www.ncbi.nlm.nih.gov/pubmed/14642641

Diedrichsen, J., Shadmehr, R., \& Ivry, R. B. (2010). The coordination of movement: Optimal feedback control and beyond. Trends in Cognitive Science, 14, 31-39.

doi: https://dx.doi.org/10.1016/j.tics.2009.11.004

PMID: https://www.ncbi.nlm.nih.gov/pubmed/20005767

Doeringer, J. A., \& Hogan, N. (1998). Intermittency in preplanned elbow movements persists in the absence of visual feedback. Journal of Neurophysiology, 80, 1787-1799. doi: https://dx.doi.org/10.1152/jn.1998.80.4.1787

PMID: https://www.ncbi.nlm.nih.gov/pubmed/9772239

Donnet, S., Bartolo, R., Fernandes, J. M., Cunha, J. P., Prado, L., \& Merchant, H. (2014). Monkeys time their pauses of movement and not their movement-kinematics during a synchronization-continuation rhythmic task. Journal of Neurophysiology, 111, 21382149.

doi: https://dx.doi.org/10.1152/jn.00802.2013

PMID: https://www.ncbi.nlm.nih.gov/pubmed/24572098

Edelman, S., \& Flash, T. (1987). A model of handwriting. Biological Cybernetics, 57, 2536.

doi: https://dx.doi.org/10.1007/BF00318713

PMID: https://www.ncbi.nlm.nih.gov/pubmed/3620543 
Evinger, C., Shaw, M. D., Peck, C. K., Manning, K. A., \& Baker R. (1984). Blinking and associated eye movements in humans, guinea pigs, and rabbits. Journal of Neurophysiology, 52, 323-339.

doi: https://dx.doi.org/10.1152/jn.1984.52.2.323

PMID: https://www.ncbi.nlm.nih.gov/pubmed/6481436

Faulkner, A., \& Rosen, S. (1999). Contributions of temporal encodings of voicing, voicelessness, fundamental frequency, and amplitude variation to audio-visual and auditory speech perception. Journal of the Acoustical Society of America, 106, 20632073.

doi: https://dx.doi.org/10.1121/1.427951

PMID: https://www.ncbi.nlm.nih.gov/pubmed/10530029

Feldman, A. G., \& Levin, M. F. (1995). The origin and use of positional frames of reference in motor control. Behavioral and Brain Sciences, 18, 723-806.

doi: https://dx.doi.org/10.1017/S0140525X0004070X

Feldman, A. G., \& Meijer, O. G. (1999). Bernstein's heritage. Discovering the right questions in motor control: Movements (1929). Motor Control, 3, 105-134.

doi: https://dx.doi.org/10.1123/mcj.3.2.105

PMID: https://www.ncbi.nlm.nih.gov/pubmed/10198145

Fitts, P. M. (1954). The information capacity of the human motor system in controlling the amplitude of movement. Journal of Experimental Psychology, 47, 381-391.

doi: https://dx.doi.org/10.1037/h0055392

PMID: https://www.ncbi.nlm.nih.gov/pubmed/13174710

Flash, T., \& Hogan, N. (1985). The coordination of arm movements: An experimentally confirmed mathematical model. Journal of Neuroscience, 5, 1688-1703.

doi: https://dx.doi.org/10.1523/JNEUROSCI.05-07-01688.1985

PMID: https://www.ncbi.nlm.nih.gov/pubmed/4020415

Flash, T., Meirovitch, Y., \& Barliya A. (2013). Models of human movement: Trajectory planning and inverse kinematics studies. Robotics and Autonomous Systems, 61, 330339.

doi: https://dx.doi.org/10.1016/j.robot.2012.09.020

Friston, K. (2011). What is optimal about motor control? Neuron, 72, 488-498.

doi: https://dx.doi.org/10.1016/j.neuron.2011.10.018

PMID: https://www.ncbi.nlm.nih.gov/pubmed/22078508

Friston, K., Mattout, J., \& Kilner, J. (2011). Action understanding and active inference.

Biological Cybernetics, 104, 137-160.

doi: https://dx.doi.org/10.1007/s00422-011-0424-z

PMID: https://www.ncbi.nlm.nih.gov/pubmed/21327826

Ganesh, G., Haruno, M., Kawato, M., \& Burdet, E. (2010). Motor memory and local minimization of error and effort, not global optimization, determine motor behavior. Journal of Neurophysiology, 104, 382-390. 
doi: https://dx.doi.org/10.1152/jn.01058.2009

PMID: https://www.ncbi.nlm.nih.gov/pubmed/20484533

Garcia, C., Prett, D., \& Morari, M. (1989). Model predictive control: Theory and practice A survey. Automatica, 25, 335-348.

doi: https://dx.doi.org/10.1016/0005-1098(89)90002-2

Gentner, D. R. (1987). Timing of skilled motor performance: Tests of the proportional duration model. Psychological Review, 94, 255-276.

doi: https://dx.doi.org/10.1037/0033-295X.94.2.255

Gibson, J. J. (1966). The senses considered as perceptual systems. Boston, MA, USA: Houghton Mifflin.

ISBN: http://www.isbnsearch.org/isbn/9780395044940

Gibson, J. J. (1986). The ecological approach to visual perception. Boston, MA, USA: Houghton Mifflin.

ISBN: http://www.isbnsearch.org/isbn/9780898599596

Gilet, E., Diard, J., \& Bessière, P. (2011). Bayesian action-perception computational model: Interaction of production and recognition of cursive letters. PLoS One, 6, e20387.

doi: https://dx.doi.org/10.1371/journal.pone.0020387

PMID: https://www.ncbi.nlm.nih.gov/pubmed/21674043

Glencross, D. J. (1975). The effects of changes in task conditions in the temporal organization of a repetitive speed skill. Ergonomics, 18, 18-27.

doi: https://dx.doi.org/10.1080/00140137508931436

Gordon, J., Ghilardi, M. F., Cooper, S. E., \& Ghez, C. (1994). Accuracy of planar reaching movements. II. Systematic extent errors resulting from inertial anisotropy. Experimental Brain Research, 99, 112-130.

doi: https://dx.doi.org/10.1007/BF00241416

PMID: https://www.ncbi.nlm.nih.gov/pubmed/7925785

Guiard, Y. (1993). On Fitts's and Hooke's laws: Simple harmonic movement in upperlimb cyclical aiming. Acta Psychologica (Amsterdam), 82, 139-159.

doi: https://dx.doi.org/10.1016/0001-6918(93)90009-G

PMID: https://www.ncbi.nlm.nih.gov/pubmed/8475763

Guigon, E., Baraduc, P., \& Desmurget, M. (2008a). Computational motor control: Feedback and accuracy. European Journal of Neuroscience, 27, 1003-1016.

doi: https://dx.doi.org/10.1111/j.1460-9568.2008.06028.x

PMID: https://www.ncbi.nlm.nih.gov/pubmed/18279368

Guigon, E., Baraduc, P., \& Desmurget, M. (2008b). Optimality, stochasticity, and variability in motor behavior. Journal of Computational Neuroscience, 24, 57-68.

doi: https://dx.doi.org/10.1007/s10827-007-0041-y

PMID: https://www.ncbi.nlm.nih.gov/pubmed/18202922 
Guigon, E. (2010). Active control of bias for the control of posture and movement. Journal of Neurophysiology, 104, 1090-1102.

doi: https://dx.doi.org/10.1152/jn.00162.2010

PMID: https://www.ncbi.nlm.nih.gov/pubmed/20538773

Guigon, E. (2011). Models and architectures for motor control: Simple or complex?. In F. Danion \& M. L. Latash (Eds.), Motor control: Theories, experiments, and applications (pp. 478-502). Oxford, England: Oxford University Press.

ISBN: http://www.isbnsearch.org/isbn/9780195395273

Guigon, E., Chafik, O., Jarrassé, N., \& Roby-Brami, A. (2019). Experimental and theoretical study of velocity fluctuations during slow movements in humans. Journal of Neurophysiology, 121, 715-727.

doi: https://dx.doi.org/10.1152/jn.00576.2018

PMID: https://www.ncbi.nlm.nih.gov/pubmed/30649981

Haken, H. (1983). Synergetics: An introduction: Nonequilibrium phase transitions and self-organization in physics, chemistry, and biology ( ${ }^{\text {rd }}$ rev. ed.). Berlin, Germany: Springer-Verlag.

doi: https://dx.doi.org/10.1007/978-3-642-88338-5

Haken, H., Kelso, J. A. S., \& Bunz, H. (1985). A theoretical model of phase transitions in human hand movements. Biological Cybernetics, 51, 347-356.

doi: https://dx.doi.org/10.1007/BF00336922

PMID: https://www.ncbi.nlm.nih.gov/pubmed/3978150

Harris, C. M., \& Wolpert, D. M. (1998). Signal-dependent noise determines motor planning. Nature, 394, 780-784.

doi: https://dx.doi.org/10.1038/29528

PMID: https://www.ncbi.nlm.nih.gov/pubmed/9723616

Harris, C. M., \& Wolpert, D. M. (2006). The main sequence of saccades optimizes speedaccuracy trade-off. Biological Cybernetics, 95, 21-29.

doi: https://dx.doi.org/10.1007/s00422-006-0064-x

PMID: https://www.ncbi.nlm.nih.gov/pubmed/16555070

Hatze, H. (1976). The complete optimization of a human motion. Mathematical Bioscience, 28, 99-135.

doi: https://dx.doi.org/10.1016/0025-5564(76)90098-5

Heath, M., Westwood, D. A., \& Binsted, G. (2004). The control of memory-guided reaching movements in peripersonal space. Motor Control, 8, 76-106.

doi: https://dx.doi.org/10.1123/mcj.8.1.76

PMID: https://www.ncbi.nlm.nih.gov/pubmed/14973339

Heath, M., Neely, K., \& Krigolson, O. (2008). Response modes influence the accuracy of monocular and binocular reaching movements. Motor Control, 12, 252-266.

doi: https://dx.doi.org/10.1123/mcj.12.3.252

PMID: https://www.ncbi.nlm.nih.gov/pubmed/18698109 
Hoff, B. (1994). A model of duration in normal and perturbed reaching movement. Biological Cybernetics, 71, 481-488.

doi: https://dx.doi.org/10.1007/BF00198466

Hoff, B., \& Arbib, M. A. (1992). A model of the effect of speed, accuracy, and perturbation on visually guided reaching. In R. Caminiti, P. B. Johnson PB, \& Y. Burnod (Eds.), Control of arm movement in space. Neurophysiological and computational approaches (pp. 285-306). Heidelberg, Germany: Springer-Verlag ISBN: http://www.isbnsearch.org/isbn/9783540550549

Hoffman, D. S., \& Strick, P. L. (1986). Step-tracking movements of the wrist in humans. I. Kinematic analysis. Journal of Neuroscience 6, 3309-3318. doi: https://dx.doi.org/10.1523/JNEUROSCI.06-11-03309.1986 PMID: https://www.ncbi.nlm.nih.gov/pubmed/3772433

Hogan, N. (1984). An organizing principle for a class of voluntary movements. Journal of Neuroscience, 4, 2745-2754.

doi: https://dx.doi.org/10.1523/JNEUROSCI.04-11-02745.1984

PMID: https://www.ncbi.nlm.nih.gov/pubmed/6502203

Hogan, N., \& Sternad, D. (2007). On rhythmic and discrete movements: Reflections, definitions and implications for motor control. Experimental Brain Research, 181, 1330.

doi: https://dx.doi.org/10.1007/s00221-007-0899-y

PMID: https://www.ncbi.nlm.nih.gov/pubmed/17530234

Hollerbach, J. M. (1981). An oscillation theory of handwriting. Biological Cybernetics, $39,139-156$.

doi: https://dx.doi.org/10.1007/BF00336740

Hove, M. J., \& Keller, P. E. (2010). Spatiotemporal relations and movement trajectories in visuomotor synchronization. Music Perception, 28, 15-26.

doi: https://dx.doi.org/10.1525/mp.2010.28.1.15

Howarth, C. I., Beggs, W. D. A., \& Bowden, J. M. (1971). The relationship between speed and accuracy of movement aimed at a target. Acta Psychologica (Amsterdam), 35, 207218.

doi: https://dx.doi.org/10.1016/0001-6918(71)90022-9

Huh, D., \& Sejnowski, T. J. (2015). Spectrum of power laws for curved hand movements. Proceedings of the National Academy of Sciences of the United States of America, 112, E3950-E3958.

doi: https://dx.doi.org/10.1073/pnas.1510208112

PMID: https://www.ncbi.nlm.nih.gov/pubmed/26150514

Huys, R., Studenka, B. E., Rheaume, N. L., Zelaznik, H. N., \& Jirsa, V. K. (2008). Distinct timing mechanisms produce discrete and continuous movements. PLoS Computational Biology, 4, e1000061.

doi: https://dx.doi.org/10.1371/journal.pcbi.1000061

PMID: https://www.ncbi.nlm.nih.gov/pubmed/18437236 
Huys, R., Fernandez, L., Bootsma, R. J., \& Jirsa, V. K. (2010). Fitts' law is not continuous in reciprocal aiming. Proceedings of the Royal Society of London B: Biological Science, 277, 1179-1184.

doi: https://dx.doi.org/10.1098/rspb.2009.1954

PMID: https://www.ncbi.nlm.nih.gov/pubmed/20018791

Huys, R., Perdikis, D., \& Jirsa V. K. (2014). Functional architectures and structured flows on manifolds: A dynamical framework for motor behavior. Psycholological Review, $121,302-336$

doi: https://dx.doi.org/10.1037/a0037014

PMID: https://www.ncbi.nlm.nih.gov/pubmed/25090422

Ijspeert, A. J., Nakanishi, J., Hoffmann, H., Pastor, P., \& Schaal, S. (2013). Dynamical movement primitives: Learning attractor models for motor behaviors. Neural Computation, 25, 328-373.

doi: https://dx.doi.org/10.1162/NECO_a 00393

PMID: https://www.ncbi.nlm.nih.gov/pubmed/23148415

Jean, F., \& Berret, B. (2017). On the duration of human movement: From self-paced to slow/fast reaches up to Fitts's law. In J.-P. Laumond, N. Mansard, \& J.-B. Lasserre (Eds.), Geometric and numerical foundations of movements (pp. 43-65). Cham, Switzerland: Springer.

doi: https://dx.doi.org/10.1007/978-3-319-51547-2_3

Jeannerod, M. (1984). The timing of natural prehension movements. Journal of Motor Behavior, 16, 235-254.

doi: https://dx.doi.org/10.1080/00222895.1984.10735319

PMID: https://www.ncbi.nlm.nih.gov/pubmed/15151851

Jirsa, V. K., \& Kelso, J. A. S. (2005). The excitator as a minimal model for the coordination dynamics of discrete and rhythmic movement generation. Journal of Motor Behavior, 37, 35-51.

doi: https://dx.doi.org/10.3200/JMBR.37.1.35-51

PMID: https://www.ncbi.nlm.nih.gov/pubmed/15642691

Jirsa, V. K., Friedrich, R., Haken, H., \& Kelso, J. A. S. (1994). A theoretical model of phase transitions in the human brain. Biological Cybernetics, 71, 27-35.

doi: https://dx.doi.org/10.1007/s004220050065

PMID: https://www.ncbi.nlm.nih.gov/pubmed/8054384

Jones, K. E., Hamilton, A. F. C., \& Wolpert, D. M. (2002). Sources of signal-dependent noise during isometric force production. Journal of Neurophysiology, 88, 1533-1544. doi: https://dx.doi.org/10.1152/jn.2002.88.3.1533

PMID: https://www.ncbi.nlm.nih.gov/pubmed/12205173

Kawato, M. (1999). Internal models for motor control and trajectory planning. Current Opinion in Neurobiology, 9, 718-727.

doi: https://dx.doi.org/10.1016/S0959-4388(99)00028-8

PMID: https://www.ncbi.nlm.nih.gov/pubmed/10607637 
Kelso, J. A. S. (1984). Phase transitions and critical behavior in human bimanual coordination. American Journal of Physiology, 246, R1000-R1004.

doi: https://dx.doi.org/10.1152/ajpregu.1984.246.6.R1000

PMID: https://www.ncbi.nlm.nih.gov/pubmed/6742155

Kelso, J. A. S. (1995). Dynamic patterns: The self-organization of brain and behavior. Cambridge, MA, USA: MIT Press.

ISBN: http://www.isbnsearch.org/isbn/9780262611312

Kelso, J. A. S., Holt, K. G., Rubin, P., \& Kugler, P. N. (1981). Patterns of human interlimb coordination emerge from the properties of non-linear, limit cycle oscillatory processes: Theory and data. Journal of Motor Behavior, 13, 226-261.

doi: https://dx.doi.org/10.1080/00222895.1981.10735251

PMID: https://www.ncbi.nlm.nih.gov/pubmed/15215072

Khan, M. A., Elliot, D., Coull, J., Chua, R., \& Lyons J. (2002). Optimal control strategies under different feedback schedules: Kinematic evidence. Journal of Motor Behavior, $34,45-57$.

doi: https://dx.doi.org/10.1080/00222890209601930

PMID: https://www.ncbi.nlm.nih.gov/pubmed/11880249

Kodl, J., Ganesh, G., \& Burdet E. (2011). The CNS stochastically selects motor plan utilizing extrinsic and intrinsic representations. PLoS One, 6, e24229.

doi: https://dx.doi.org/10.1371/journal.pone.0024229

PMID: https://www.ncbi.nlm.nih.gov/pubmed/21912679

Krüger, M., Eggert, T., \& Straube, A. (2011). Joint angle variability in the time course of reaching movements. Clinical Neurophysiology, 122, 759-766.

doi: https://dx.doi.org/10.1016/j.clinph.2010.10.003

PMID: https://www.ncbi.nlm.nih.gov/pubmed/21030301

Krüger, M., Straube, A., \& Eggert, T. (2017). The propagation of movement variability in time: A methodological approach for discrete movements with multiple degrees of freedom. Frontiers in Computational Neuroscience, 11, 93.

doi: https://dx.doi.org/10.3389/fncom.2017.00093

PMID: https://www.ncbi.nlm.nih.gov/pubmed/29081743

Kugler, P. N., Kelso, J. A. S., \& Turvey, M. T. (1980). On the concept of coordinative structures as dissipative structures: I. Theoretical lines of convergence. In G. E. Stelmach, \& J. Requin (Eds.), Tutorials in motor behavior, Vol 1 (pp. 3-47). Amsterdam, The Netherlands: North-Holland. doi: https://dx.doi.org/10.1016/S0166-4115(08)61936-6

Lacquaniti, F., Terzuolo, C., \& Viviani, P. (1983). The law relating the kinematic and figural aspects of drawing movements. Acta Psychologica (Amsterdam), 54, 115-130. doi: https://dx.doi.org/10.1016/0001-6918(83)90027-6

PMID: https://www.ncbi.nlm.nih.gov/pubmed/6666647 
Lakie, M., Caplan, N., \& Loram, I. D. (2003). Human balancing of an inverted pendulum with a compliant linkage: Neural control by anticipatory intermittent bias. Journal of Physiology (London), 551, 357-370.

doi: https://dx.doi.org/10.1113/jphysiol.2002.036939

PMID: https://www.ncbi.nlm.nih.gov/pubmed/12832494

Lashley, K. S. (1951). The problem of serial order in behavior. In L. A. Jeffress (Ed.), Cerebral mechanisms of behavior (pp. 112-136). New York, NY, USA: John Wiley \& Sons.

Levy-Tzedek, S., Ben Tov, M., \& Karniel, A. (2011). Rhythmic movements are larger and faster but with the same frequency on removal of visual feedback. Journal of Neurophysiology, 106, 2120-2126.

doi: https://dx.doi.org/10.1152/jn.00266.2011

PMID: https://www.ncbi.nlm.nih.gov/pubmed/21813746

Liu, D., \& Todorov, E. (2007). Evidence for the flexible sensorimotor strategies predicted by optimal feedback control. Journal of Neuroscience, 27, 9354-9368.

doi: https://dx.doi.org/10.1523/JNEUROSCI.1110-06.2007

PMID: https://www.ncbi.nlm.nih.gov/pubmed/17728449

Loram, I. D., Kelly, S. M., \& Lakie, M. (2001). Human balancing of an inverted pendulum: Is sway size controlled by ankle impedance? Journal of Physiology (London), 532, 879-891.

doi: https://dx.doi.org/10.1111/j.1469-7793.2001.0879e.x

PMID: https://www.ncbi.nlm.nih.gov/pubmed/11313453

Loram, I. D., \& Lakie, M. (2002). Direct measurement of human ankle stiffness during quiet standing: The intrinsic mechanical stiffness is insufficient for stability. Journal of Physiology (London), 545, 1041-1053.

doi: https://dx.doi.org/10.1113/jphysiol.2002.025049

PMID: https://www.ncbi.nlm.nih.gov/pubmed/12482906

MacKenzie, C. L., Marteniuk, R. G., Dugas, C., Liske, D., \& Eckmeier, B. (1987). Three dimensional movement trajectories in Fitts' task: Implications for control. Quarterly Journal of Experimental Psychology A, 39, 629-647.

doi: https://dx.doi.org/10.1080/14640748708401806

Martin, V., Reimann, H., \& Schöner, G. (2019). A process account of the uncontrolled manifold structure of joint space variance in pointing movements. Biological Cybernetics, 113, 293-307.

doi: https://dx.doi.org/10.1007/s00422-019-00794-w

PMID: https://www.ncbi.nlm.nih.gov/pubmed/30771072

Mechsner, F., Kerzel, D., Knoblich, G., \& Prinz, W. (2001). Perceptual basis of bimanual coordination. Nature, 414, 69-73.

doi: https://dx.doi.org/10.1038/35102060

PMID: https://www.ncbi.nlm.nih.gov/pubmed/11689944 
Meijer, O. G., \& Bruijn, S. M. (2007). The loyal dissident: N.A. Bernstein and the doubleedged sword of Stalinism. Journal of the History of the Neurosciences, 16, 206-224. doi: https://dx.doi.org/10.1080/09647040600720979

PMID: https://www.ncbi.nlm.nih.gov/pubmed/17365565

Messier, J., \& Kalaska, J. F. (1999). Comparison of variability of initial kinematics and endpoints of reaching movements. Experimental Brain Research, 125, 139-152.

doi: https://dx.doi.org/10.1007/s002210050669

PMID: https://www.ncbi.nlm.nih.gov/pubmed/10204767

Meulenbroek, R. G., Rosenbaum, D. A., Thomassen, A. J., Loukopoulos, L. D., \& Vaughan, J. (1996). Adaptation of a reaching model to handwriting: How different effectors can produce the same written output, and other results. Psychological Research, 59, 64-74.

doi: https://dx.doi.org/10.1007/BF00419834

PMID: https://www.ncbi.nlm.nih.gov/pubmed/8693052

Meyer, D. E., Abrams, R. A., Kornblum, S., Wright, C. E., \& Smith J. E. K. (1988). Optimality in human motor performance: Ideal control of rapid aimed movement. Psychological Review, 95, 340-370.

doi: https://dx.doi.org/10.1037/0033-295x.95.3.340

PMID: https://www.ncbi.nlm.nih.gov/pubmed/3406245

Mitrovic, D., Klanke, S., Osu, R., Kawato, M., \& Vijayakumar, S. (2010). A computational model of limb impedance control based on principles of internal model uncertainty. PLoS One, 5, e13601.

doi: https://dx.doi.org/10.1371/journal.pone.0013601

PMID: https://www.ncbi.nlm.nih.gov/pubmed/21049061

Mohan, V., \& Morasso, P. (2011). Passive motion paradigm: An alternative to optimal control. Frontiers in Neurorobotics, 5, 4.

doi: https://dx.doi.org/10.3389/fnbot.2011.00004

PMID: https://www.ncbi.nlm.nih.gov/pubmed/22207846

Mohan, V., Bhat, A., \& Morasso, P. (2019). Muscleless motor synergies and actions without movements: From motor neuroscience to cognitive robotics. Physics of Life Review, 30, 89-111.

doi: https://dx.doi.org/10.1016/j.plrev.2018.04.005

Morasso, P., \& Mussa-Ivaldi, F. A. (1982). Trajectory formation and handwriting: A computational model. Biological Cybernetics, 45, 131-142.

doi: https://dx.doi.org/10.1007/BF00335240

PMID: https://pubmed.ncbi.nlm.nih.gov/7138957

Morasso, P., Mussa-Ivaldi, F. A., \& Ruggiero, C. (1983). How a discontinuous mechanism can produce continuous patterns in trajectory formation and handwriting. Acta Psychologica (Amsterdam), 54, 83-98.

doi: https://dx.doi.org/10.1016/0001-6918(83)90025-2 
Morasso, P. G., \& Schieppati, M. (1999). Can muscle stiffness alone stabilize upright standing? Journal of Neurophysiology, 82, 1622-1626.

doi: https://dx.doi.org/10.1152/jn.1999.82.3.1622

PMID: https://www.ncbi.nlm.nih.gov/pubmed/10482776

Morasso, P. G., \& Sanguineti, V. (2002). Ankle muscle stiffness alone cannot stabilize balance during quiet standing. Journal of Neurophysiology, 88, 2157-2162.

doi: https://dx.doi.org/10.1152/jn.2002.88.4.2157

PMID: https://www.ncbi.nlm.nih.gov/pubmed/12364538

Mosier, K. M., Scheidt, R. A., Acosta, S., \& Mussa-Ivaldi, F. A. (2005). Remapping hand movements in a novel geometrical environment. Journal of Neurophysiology, 94, 4362-4372.

doi: https://dx.doi.org/10.1152/jn.00380.2005

PMID: https://www.ncbi.nlm.nih.gov/pubmed/16148276

Nelson, W. L. (1983). Physical principles for economies of skilled movements. Biological Cybernetics, 46, 135-147.

doi: https://dx.doi.org/10.1007/BF00339982

PMID: https://www.ncbi.nlm.nih.gov/pubmed/6838914

Ostry, D. J., \& Feldman, A. G. (2003). A critical evaluation of the force control hypothesis in motor control. Experimental Brain Research, 153, 275-288.

doi: https://dx.doi.org/10.1007/s00221-003-1624-0

PMID: https://www.ncbi.nlm.nih.gov/pubmed/14610628

Osu, R., Morishige, K., Nakanishi, J., Miyamoto, H., \& Kawato, M. (2015). Practice reduces task relevant variance modulation and forms nominal trajectory. Scientific Reports, 5, 17659.

doi: https://dx.doi.org/10.1038/srep17659

PMID: https://www.ncbi.nlm.nih.gov/pubmed/26639942

Pashler, H. (1994). Dual-task interference in simple tasks: Data and theory. Psychological Bulletin, 116, 220-244

doi: https://dx.doi.org/10.1037/0033-2909.116.2.220

PMID: https://pubmed.ncbi.nlm.nih.gov/7972591

Pélisson, D., Prablanc, C., Goodale, M. A., \& Jeannerod, M. (1986). Visual control of reaching movements without vision of the limb. II. Evidence of fast unconscious processes correcting the trajectory of the hand to the final position of a double-step stimulus. Experimental Brain Research, 62, 303-311.

doi: https://dx.doi.org/10.1007/BF00238849

PMID: https://www.ncbi.nlm.nih.gov/pubmed/3709715

Perdikis, D., Huys, R., \& Jirsa, V. K. (2011). Time scale hierarchies in the functional organization of complex behaviors. PLoS Computational Biology, 7, e1002198.

doi: https://dx.doi.org/10.1371/journal.pcbi.1002198

PMID: https://www.ncbi.nlm.nih.gov/pubmed/21980278 
Poletti, C., Sleimen-Malkoun, R., Temprado, J.-J., \& Lemaire, P. (2015). Older and younger adults' strategies in sensorimotor tasks: Insights from Fitts' pointing task. Journal of Experimental Psychology: Human Perception \& Performance, 41, 542-555. doi: https://dx.doi.org/10.1037/xhp0000033

PMID: https://www.ncbi.nlm.nih.gov/pubmed/25751042

Port, N. L., Lee, D., Dassonville, P., \& Georgopoulos, A. P. (1997). Manual interception of moving targets: I. Performance and movement initiation. Experimental Brain Research, 116, 406-420.

doi: https://dx.doi.org/10.1007/PL00005769

PMID: https://www.ncbi.nlm.nih.gov/pubmed/9372290

Powers, A. S., Basso, M. A., \& Evinger, C. (2013). Blinks slow memory-guided saccades. Journal of Neurophysiology, 109, 734-741.

doi: https://dx.doi.org/10.1152/jn.00746.2012

PMID: https://www.ncbi.nlm.nih.gov/pubmed/23155174

Qian, N., Jiang, Y., Jiang, Z. P., \& Mazzoni, P. (2013). Movement duration, Fitts's law, and an infinite-horizon optimal feedback control model for biological motor systems. Neural Computation, 25, 697-724.

doi: https://dx.doi.org/10.1162/NECO a 00410

PMID: https://www.ncbi.nlm.nih.gov/pubmed/23272916

Rieger, M. (2007). The role of goal representations in moving to temporal and spatial targets. Quarterly Journal of Experimental Psychology (Hove), 60, 262-288.

doi: https://dx.doi.org/10.1080/17470210600760250

PMID: https://www.ncbi.nlm.nih.gov/pubmed/17455058

Rigoux, L., \& Guigon, E. (2012). A model of reward- and effort-based optimal decision making and motor control. PLoS Computational Biology, 8, e1002716

doi: https://dx.doi.org/10.1371/journal.pcbi.1002716

PMID: https://www.ncbi.nlm.nih.gov/pubmed/23055916

Ronsse, R., Sternad, D., \& Lefèvre, P. (2009). A computational model for rhythmic and discrete movements in uni- and bimanual coordination. Neural Computation, 21, 13351370 .

doi: https://dx.doi.org/10.1162/neco.2008.03-08-720

PMID: https://www.ncbi.nlm.nih.gov/pubmed/19018700

Saeb, S., Weber, C., \& Triesch, J. (2011). Learning the optimal control of coordinated eye and head movements. PLoS Computational Biology, 7, e1002253.

doi: https://dx.doi.org/10.1371/journal.pcbi.1002253

PMID: https://www.ncbi.nlm.nih.gov/pubmed/22072953

Saha, D. J., \& Morasso, P. (2012). Stabilization strategies for unstable dynamics. PLoS One, 7, e30301.

doi: https://dx.doi.org/10.1371/journal.pone.0030301

PMID: https://www.ncbi.nlm.nih.gov/pubmed/22279580 
Saito, H., Tsubone, T., \& Wada, Y. (2006). Can human isochrony be explained by a computational theory? Annual International Conference of the IEEE Engineering in Medicine and Biology Society, 2006, 4494-4497.

doi: https://dx.doi.org/10.1109/IEMBS.2006.260819

PMID: https://www.ncbi.nlm.nih.gov/pubmed/17946633

Sainburg, R. L, \& Schaefer, S. Y. (2004). Interlimb differences in control of movement extent. Journal of Neurophysiology, 92, 1374-1383.

doi: https://dx.doi.org/10.1152/jn.00181.2004

PMID: https://www.ncbi.nlm.nih.gov/pubmed/15115793

Salmond, L. H., Davidson, A. D., \& Charles, S. K. (2017). Proximal-distal differences in movement smoothness reflect differences in biomechanics. Journal of Neurophysiology, 117, 1239-1257.

doi: https://dx.doi.org/10.1152/jn.00712.2015

PMID: https://www.ncbi.nlm.nih.gov/pubmed/28003410

Saltzman, E., \& Kelso, J. A. S. (1987). Skilled actions: A task-dynamic approach. Psychological Review, 94, 84-106.

doi: https://dx.doi.org/10.1037/0033-295X.94.1.84

PMID: https://www.ncbi.nlm.nih.gov/pubmed/3823306

Sarlegna, F., Blouin, J., Bresciani, J.-P., Bourdin, C., Vercher, J.-L., \& Gauthier, G. M. (2003). Target and hand position information in the online control of goal-directed arm movements. Experimental Brain Research, 151, 524-535.

doi: https://dx.doi.org/10.1007/s00221-003-1504-7

PMID: https://www.ncbi.nlm.nih.gov/pubmed/12830346

Schaal, S., Ijspeert, A., \& Billard, A. (2003). Computational approaches to motor learning by imitation. Philosophical transactions of the Royal Society of London. Series B, Biological sciences, 358, 537-547.

doi: https://doi.org/10.1098/rstb.2002.1258

PMID: https://pubmed.ncbi.nlm.nih.gov/12689379

Schaal, S., Kotosaka, S., \& Sternad, D. (2000). Nonlinear dynamical systems as movement primitives. In Proceeding of the IEEE-RAS International Conference on Humanoid Robots. Piscataway, NJ, USA: IEEE Press.

Schaal, S., Sternad, D., Osu, R., \& Kawato, M. (2004). Rhythmic arm movement is not discrete. Nature Neuroscience, 7, 1136-1143.

doi: https://dx.doi.org/10.1038/nn1322

PMID: https://www.ncbi.nlm.nih.gov/pubmed/15452580

Schaal, S., Mohajerian, P., \& Ijspeert, A. (2007). Dynamics systems vs. optimal control--a unifying view. Progress in Brain Research, 165, 425-445.

doi: https://dx.doi.org/10.1016/S0079-6123(06)65027-9

PMID: https://www.ncbi.nlm.nih.gov/pubmed/17925262 
Schmidt, R. A., Zelaznik, H. N., Hawkins, B., Franck, J. S., \& Quinn, J. T. (1979). Motoroutput variability: A theory for the accuracy of rapid motor acts. Psychological Review, $86,415-451$.

doi: https://dx.doi.org/10.1037/0033-295X.86.5.415

PMID: https://www.ncbi.nlm.nih.gov/pubmed/504536

Scholz, J. P., Kelso, J. A. S., \& Schöner, G. (1987). Nonequilibrium phase transitions in coordinated biological motion: Critical slowing down and switching time. Physics Letters A, 123, 390-394.

doi: https://dx.doi.org/10.1016/0375-9601(87)90038-7

Scholz, J. P., \& Schöner, G. (1999). The uncontrolled manifold concept: Identifying control variables for a functional task. Experimental Brain Research, 126, 289-306. doi: https://dx.doi.org/10.1007/s002210050738 PMID: https://www.ncbi.nlm.nih.gov/pubmed/10382616

Schöner, G. (1990). A dynamic theory of coordination of discrete movement. Biological Cybernetics, 63, 257-270.

doi: https://dx.doi.org/10.1007/BF00203449

PMID: https://www.ncbi.nlm.nih.gov/pubmed/2207200

Schöner, G. (1994). From interlimb coordination to trajectory formation: Common dynamical principles. In S. P. Swinnen, H. Heuer, J. Massion, \& P. Casaer (Eds.), Interlimb coordination: Neural, dynamical and cognitive constraints (pp. 339-368). San Diego, CA: Academic Press.

doi: https://dx.doi.org/10.1016/B978-0-12-679270-6.50022-X

Scott, S. H. (2004). Optimal feedback control and the neural basis of volitional motor control. Nature Reviews Neuroscience, 5, 532-546.

doi: https://dx.doi.org/10.1038/nrn1427

PMID: https://www.ncbi.nlm.nih.gov/pubmed/15208695

Selen, L. P., Beek, P. J., \& van Dieën J. H. (2006). Impedance is modulated to meet accuracy demands during goal-directed arm movements. Experimental Brain Research, $172,129-138$.

doi: https://dx.doi.org/10.1007/s00221-005-0320-7

PMID: https://www.ncbi.nlm.nih.gov/pubmed/16372169

Shadmehr, R., \& Mussa-Ivaldi, F. A. (1994). Adaptive representation of dynamics during learning a motor task. Journal of Neuroscience, 14, 3208-3224.

doi: https://dx.doi.org/10.1523/JNEUROSCI.14-05-03208.1994

PMID: https://www.ncbi.nlm.nih.gov/pubmed/8182467

Shadmehr, R., Orban de Xivry, J. J., Xu-Wilson, M., \& Shih, T. Y. (2010). Temporal discounting of reward and the cost of time in motor control. Journal of Neuroscience, 30, 10507-10516.

doi: https://dx.doi.org/10.1523/JNEUROSCI.1343-10.2010

PMID: https://www.ncbi.nlm.nih.gov/pubmed/20685993 
Shmuelof, L., Krakauer, J. W., \& Mazzoni, P. (2012). How is a motor skill learned? Change and invariance at the levels of task success and trajectory control. Journal of Neurophysiology, 108, 578-594.

doi: https://dx.doi.org/10.1152/jn.00856.2011

PMID: https://www.ncbi.nlm.nih.gov/pubmed/22514286

Singer, Y., \& Tishby, N. (1994). Dynamical encoding of cursive handwriting. Biological Cybernetics, 71, 227-238.

doi: https://dx.doi.org/10.1007/s004220050085

PMID: https://www.ncbi.nlm.nih.gov/pubmed/7918801

Sleimen-Malkoun, R., Temprado, J.-J., \& Berton, E. (2013). Age-related changes of movement patterns in discrete Fitts' task. BMC Neuroscience, 14, 145.

doi: https://dx.doi.org/10.1186/1471-2202-14-145

PMID: https://www.ncbi.nlm.nih.gov/pubmed/24228864

Slifkin, A. B., \& Eder, J. R. (2012). Amplitude requirements, visual information, and the spatial structure of movement. Experimental Brain Research, 220, 297-310.

doi: https://dx.doi.org/10.1007/s00221-012-3138-0

PMID: https://www.ncbi.nlm.nih.gov/pubmed/22718148

Smith, A., Goffman, L., Zelaznik, H. N., Ying, G. S., \& McGillem, C. (1995). Spatiotemporal stability and patterning of speech movement sequences. Experimental Brain Research, 104, 493-501.

doi: https://dx.doi.org/10.1007/BF00231983

PMID: https://www.ncbi.nlm.nih.gov/pubmed/7589300

Stengel, R. F. (1994). Optimal control and estimation. New York, NY, USA: Dover.

ISBN: http://www.isbnsearch.org/isbn/9780486682006

Sternad, D. (1998). Hot topics in motor control and learning. A dynamic systems perspective to perception and action. Research Quarterly for Exercise and Sport, 69, 319-325.

doi: https://dx.doi.org/10.1080/02701367.1998.10607705

PMID: https://www.ncbi.nlm.nih.gov/pubmed/9864749

Sternad, D. (2000). Debates in dynamics: A dynamical systems perspective on action and perception. Human Movement Science, 19, 407-423.

doi: https://dx.doi.org/10.1016/S0167-9457(00)00024-5

Sternad, D., Marino, H., Charles, S.K., Duarte, M., Dipietro, L., \& Hogan, N. (2013). Transitions between discrete and rhythmic primitives in a unimanual task. Frontiers in Computational Neuroscience, 7, 90.

doi: https://dx.doi.org/10.3389/fncom.2013.00090

PMID: https://www.ncbi.nlm.nih.gov/pubmed/23888139

Strogatz, S. H. (1994). Nonlinear dynamics and chaos. With applications to physics, biology, chemistry, and engineering. Cambridge, MA, USA: Perseus.

ISBN: http://www.isbnsearch.org/isbn/9780738204536 
Tanaka, H., Krakauer, J. W., \& Qian, N. (2006). An optimization principle for determining movement duration. Journal of Neurophysiology, 95, 3875-3886.

doi: https://dx.doi.org/10.1152/jn.00751.2005

PMID: https://www.ncbi.nlm.nih.gov/pubmed/16571740

Temprado, J.-J., Sleimen-Malkoun, R., Lemaire, P., Rey-Robert, B., Retornaz, F., \& Berton, E. (2013). Aging of sensorimotor processes: A systematic study in Fitts' task. Experimental Brain Research, 228, 105-116.

doi: https://dx.doi.org/10.1007/s00221-013-3542-0

PMID: https://www.ncbi.nlm.nih.gov/pubmed/23649969

Teulings, H.-L., \& Stelmach, G. E. (1991). Control of stroke size, peak acceleration, and stroke duration in parkinsonian handwriting. Human Movement Science, 10, 315-334. doi: https://dx.doi.org/10.1016/0167-9457(91)90010-U

Todorov, E., \& Jordan, M. I. (2002). Optimal feedback control as a theory of motor coordination. Nature Neuroscience, 5, 1226-1235.

doi: https://dx.doi.org/10.1038/nn963

PMID: https://www.ncbi.nlm.nih.gov/pubmed/12404008

Todorov, E. (2002). Cosine tuning minimizes motor errors. Neural Computation, 14, $1233-1260$.

doi: https://dx.doi.org/10.1162/089976602753712918

PMID: https://www.ncbi.nlm.nih.gov/pubmed/12020444

Todorov, E. (2004). Optimality principles in sensorimotor control. Nature Neuroscience, 7, 907-915.

doi: https://dx.doi.org/10.1038/nn1309

PMID: https://www.ncbi.nlm.nih.gov/pubmed/15332089

Todorov, E. (2005). Stochastic optimal control and estimation methods adapted to the noise characteristics of the sensorimotor system. Neural Computation, 17, 1084-1108. doi: https://dx.doi.org/10.1162/0899766053491887

PMID: https://www.ncbi.nlm.nih.gov/pubmed/15829101

Torres, E. B., \& Andersen, R. (2006). Space-time separation during obstacle-avoidance learning in monkeys. Journal of Neurophysiology, 96, 2613-2632.

doi: https://dx.doi.org/10.1152/jn.00188.2006

doi: https://www.ncbi.nlm.nih.gov/pubmed/16855113

Turvey, M. T. (1977). Preliminaries to a theory of action with reference to vision. In R. Shaw \& J. Bransford (Eds.), Perceiving, acting and knowing: Toward an ecological psychology (pp. 211-265). Hillsdale, NJ, USA: Lawrence Erlbaum Associates.

Uno, Y., Kawato, M., \& Suzuki, R. (1989). Formation and control of optimal trajectory in human multijoint arm movement - minimum torque change model. Biological Cybernetics, 61, 89-101. doi: https://dx.doi.org/10.1007/BF00204593

PMID: https://www.ncbi.nlm.nih.gov/pubmed/2742921 
Valdez, A. B., \& Amazeen E. L. (2008). Using 1/f noise to examine planning and control in a discrete aiming task. Experimental Brain Research, 187, 303-319.

doi: https://dx.doi.org/10.1007/s00221-008-1305-0

PMID: https://www.ncbi.nlm.nih.gov/pubmed/18283444

Vallbo, A. B., \& Wessberg, J. (1993). Organization of motor output in slow finger movements. Journal of Physiology (London), 469, 673-691.

doi: https://dx.doi.org/10.1113/jphysiol.1993.sp019837

PMID: https://www.ncbi.nlm.nih.gov/pubmed/8271223

van Beers, R. J. (2008). Saccadic eye movements minimize the consequences of motor noise. PLoS One, 3, e2070.

doi: https://dx.doi.org/10.1371/journal.pone.0002070

PMID: https://www.ncbi.nlm.nih.gov/pubmed/18446209

van Beers, R. J., Haggard, P., \& Wolpert, D. M. (2004). The role of execution noise in movement variability. Journal of Neurophysiology, 91, 1050-1063.

doi: https://dx.doi.org/10.1152/jn.00652.2003

PMID: https://www.ncbi.nlm.nih.gov/pubmed/14561687

van Beers, R. J., Brenner, E., \& Smeets, J. B. J. (2013). Random walk of motor planning in task-irrelevant dimensions. Journal of Neurophysiology, 109, 969-977.

doi: https://dx.doi.org/10.1152/jn.00706.2012

PMID: https://www.ncbi.nlm.nih.gov/pubmed/23175799

van der Helm, F. C. T., \& Rozendaal, L. A. (2000). Musculoskeletal systems with intrinsic and proprioceptive feedback. In J. M. Winters \& P. E. Crago (Eds), Biomechanics and neural control of posture and movement (pp. 164-174). New York, NY, USA: Springer.

doi: https://dx.doi.org/10.1007/978-1-4612-2104-3_11

van Mourik, A. M., \& Beek, P. J. (2004). Discrete and cyclical movements: Unified dynamics or separate control? Acta Psychologica (Amsterdam), 117, 121-138.

doi: https://dx.doi.org/10.1016/j.actpsy.2004.06.001

PMID: https://www.ncbi.nlm.nih.gov/pubmed/15464010

Viviani, P., \& Cenzato, M. (1985). Segmentation and coupling in complex movements. Journal of Experimental Psychology: Human Perception \& Performance, 11, 828-845. doi: https://dx.doi.org/10.1037/0096-1523.11.6.828

PMID: https://www.ncbi.nlm.nih.gov/pubmed/2934511

Viviani, P., \& McCollum, G. (1983). The relation between linear extent and velocity in drawing movements. Neuroscience, 10, 211-218. doi: https://dx.doi.org/10.1016/0306-4522(83)90094-5

PMID: https://www.ncbi.nlm.nih.gov/pubmed/6646421

Viviani, P., \& Schneider, R. (1991). A developmental study of the relationship between geometry and kinematics of drawing movements. Journal of Experimental Psychology: Human Perception \& Performance, 17, 198-218. doi: https://dx.doi.org/10.1037/0096-1523.17.1.198 
PMID: https://www.ncbi.nlm.nih.gov/pubmed/1826312

Viviani, P., \& Terzuolo, C. (1982). Trajectory determines movement dynamics. Neuroscience, 7, 431-437. doi: https://dx.doi.org/10.1016/0306-4522(82)90277-9

PMID: https://www.ncbi.nlm.nih.gov/pubmed/7078732

von Holst, E., \& Mittelstaedt, H. (1973). The reafference principle. In R. D. Martin (Ed. \& Trans.), The behavioral physiology of animals and man: Selected papers of E. von Holst (Vol. 1). Coral Gables, FL, USA: University of Miami Press. (Original work published 1950).

ISBN: http://www.isbnsearch.org/isbn/9780870242618

Wada, Y., \& Kawato, M. (1995). A theory for cursive handwriting based on the minimization principle. Biological Cybernetics, 73, 3-13.

doi: https://dx.doi.org/10.1007/s004220050158

PMID: https://www.ncbi.nlm.nih.gov/pubmed/7654848

Walter, A. M., \& Rieger, M. (2012). Target- and effect-directed actions towards temporal goals: Similar mechanisms? Journal of Experimental Psychology: Human Perception \& Performance, 38, 958-974.

doi: https://dx.doi.org/10.1037/a0026792

PMID: https://www.ncbi.nlm.nih.gov/pubmed/22686693

Walsh, B., \& Smith, A. (2012). Basic parameters of articulatory movements and acoustics in individuals with Parkinson's disease. Movement Disorders, 27, 843-850.

doi: https://dx.doi.org/10.1002/mds.24888

PMID: https://www.ncbi.nlm.nih.gov/pubmed/22729986

Warren, W. H. (2006). The dynamics of perception and action. Psychological Review, $113,358-389$.

doi: https://dx.doi.org/10.1037/0033-295X.113.2.358

PMID: https://www.ncbi.nlm.nih.gov/pubmed/16637765

Wolpert, D. W., \& Ghahramani, Z. (2000). Computational principles of movement neuroscience. Nature Neuroscience, 3(Suppl), 1212-1217.

doi: https://dx.doi.org/10.1038/81497

PMID: https://www.ncbi.nlm.nih.gov/pubmed/11127840

Wolpert, D. M., Ghahramani, Z., \& Jordan, M. I. (1995). An internal model for sensorimotor integration. Science, 269, 1880-1882.

doi: https://dx.doi.org/10.1126/science.7569931

PMID: https://www.ncbi.nlm.nih.gov/pubmed/7569931

Worringham, C. J. (1991). Variability effects on the internal structure of rapid aiming movement. Journal of Motor Behavior, 23, 75-85.

doi: https://dx.doi.org/10.1080/00222895.1991.9941595

PMID: https://www.ncbi.nlm.nih.gov/pubmed/14766533 
Wright, C. E, \& Meyer, D. E. (1983). Conditions for a linear speed-accuracy trade-off in aimed movements. Quarterly Journal of Experimental Psychology A, 35, 279-296.

doi: https://dx.doi.org/10.1080/14640748308402134

PMID: https://www.ncbi.nlm.nih.gov/pubmed/6571311

Zago, M., Matic, A., Flash, T., Gomez-Marin, A., \& Lacquaniti, F. (2018). The speedcurvature power law of movements: A reappraisal. Experimental Brain Research, 236, 69-82.

doi: https://dx.doi.org/10.1007/s00221-017-5108-Z

PMI 
A

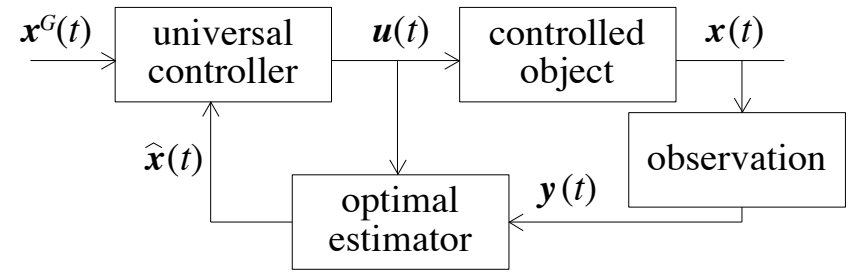

B

C

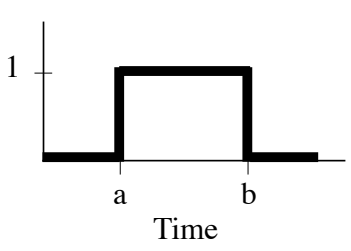

$$
\boldsymbol{x}^{G}(t)
$$

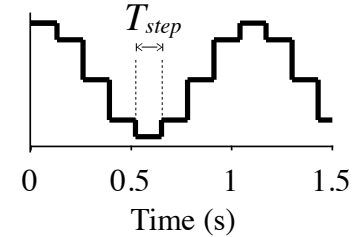

Figure 1 

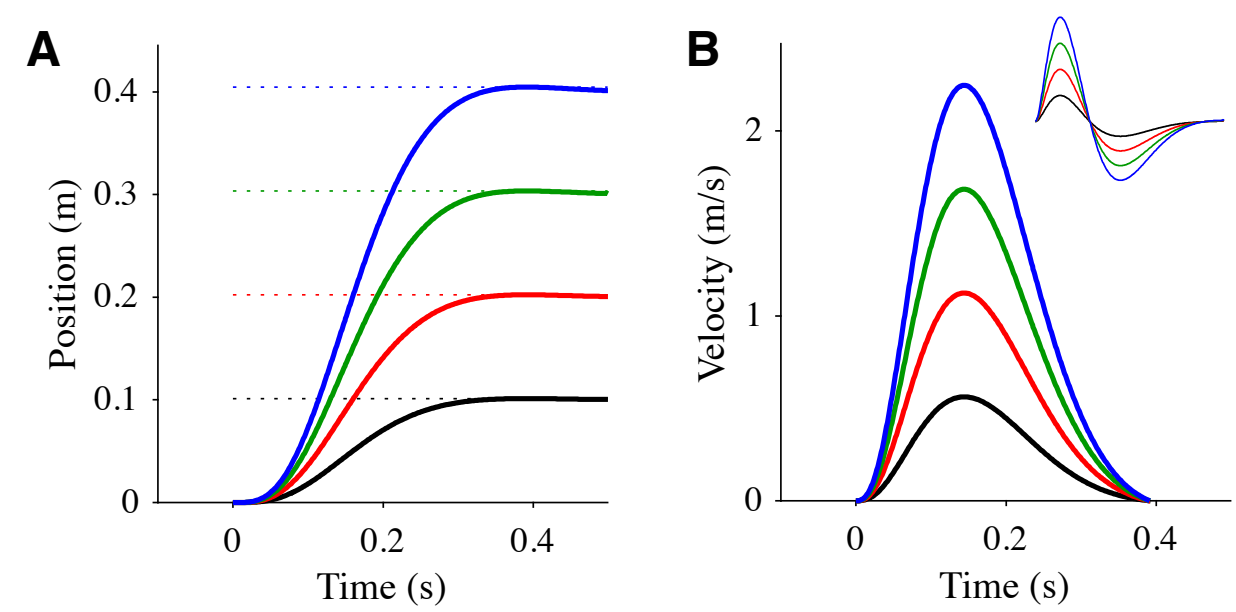

Figure 2 

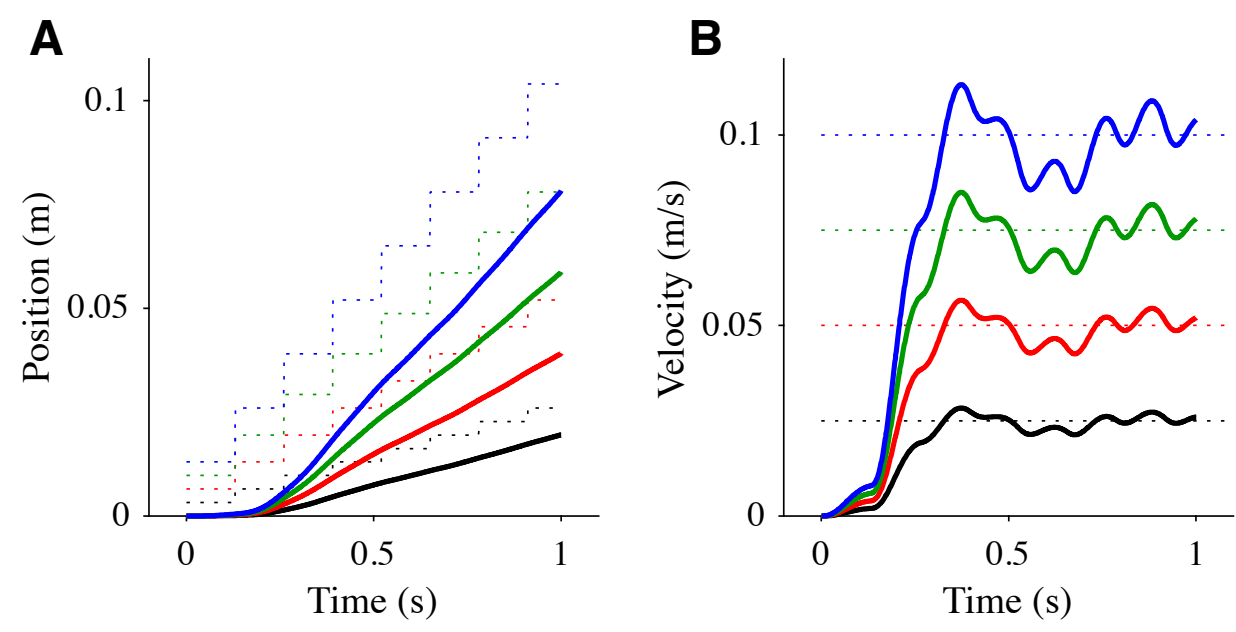

Figure 3 
A

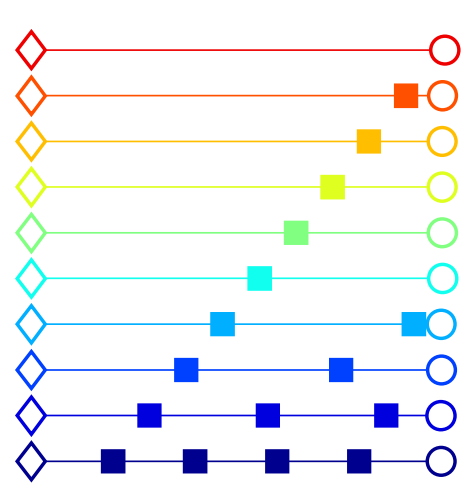

B

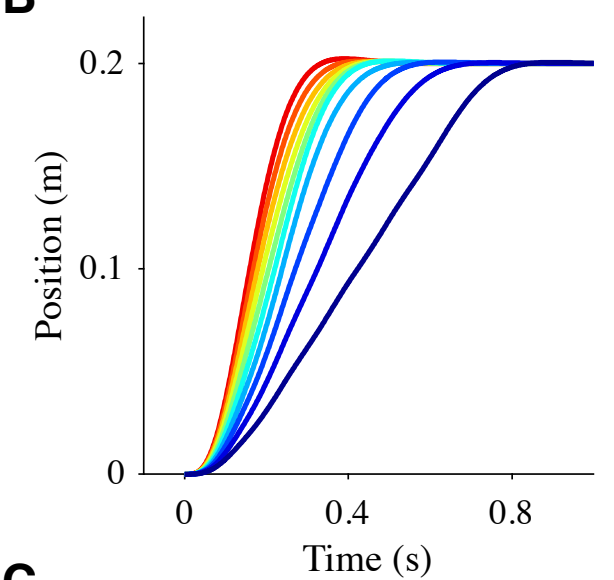

C

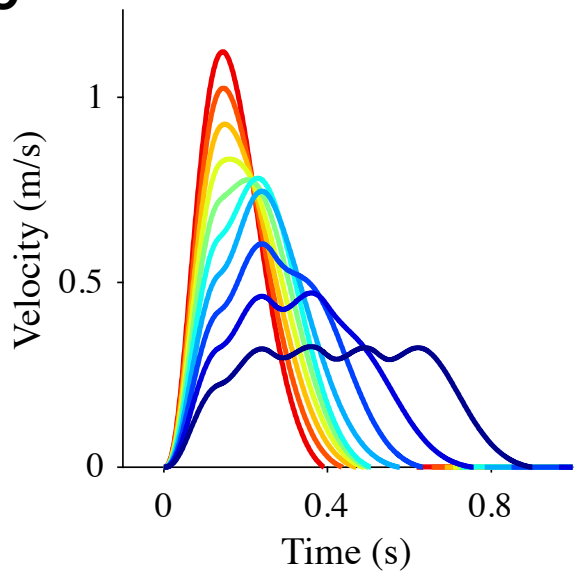

D

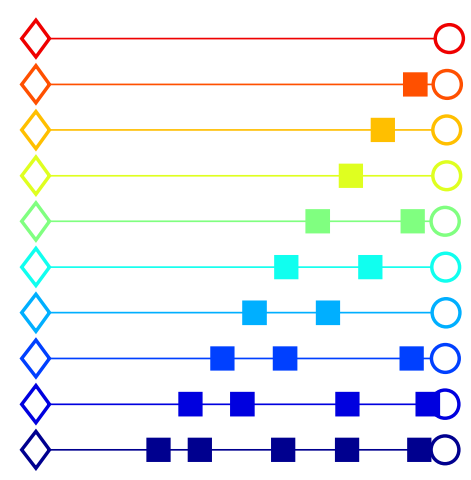

E

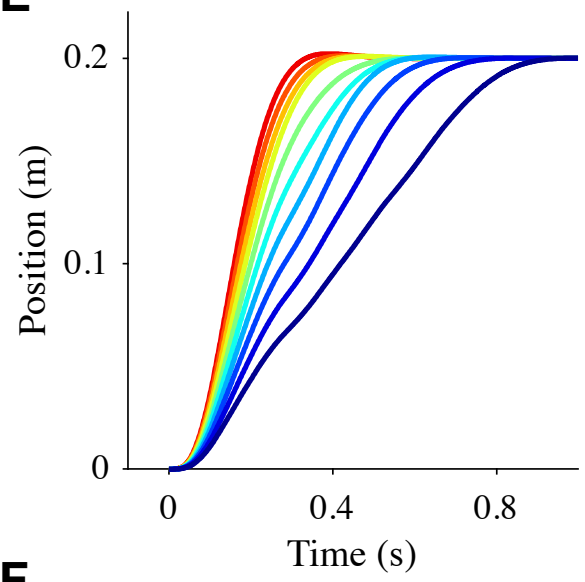

F

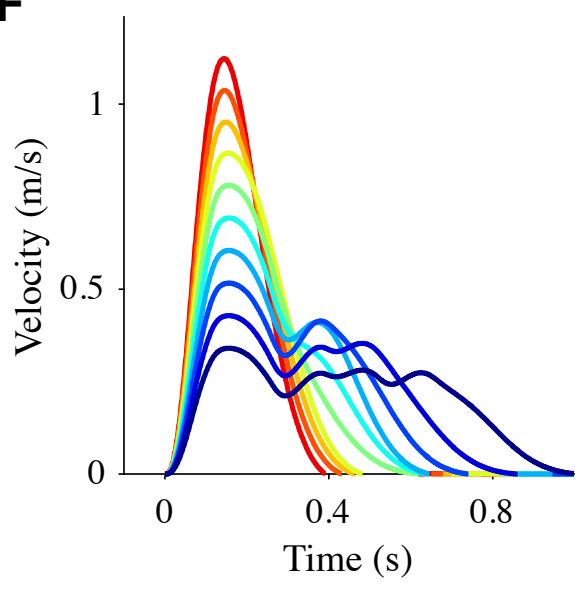

Figure 4 
A $\square$
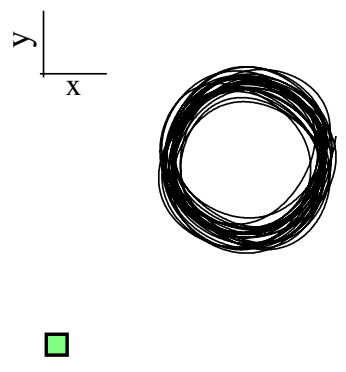

B

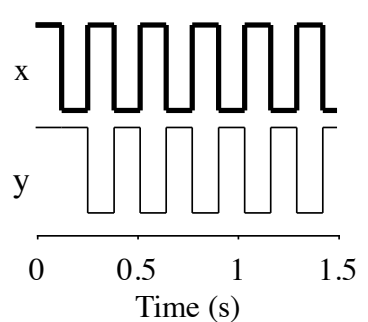

- C

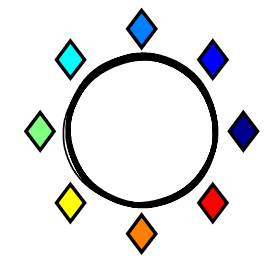

$\square$

D

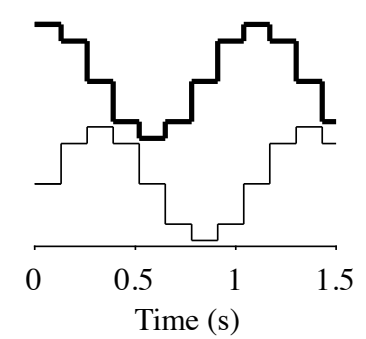

E

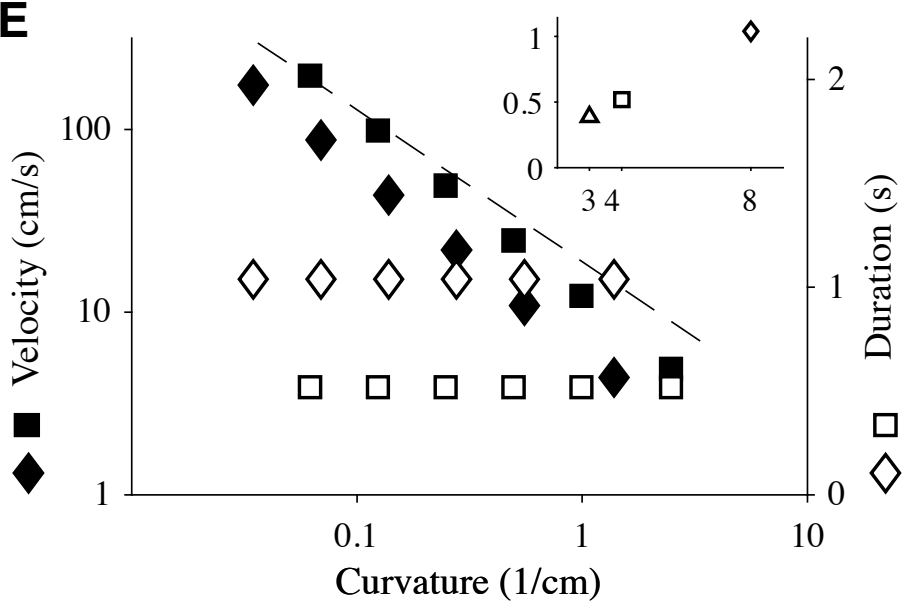

Figure 5 

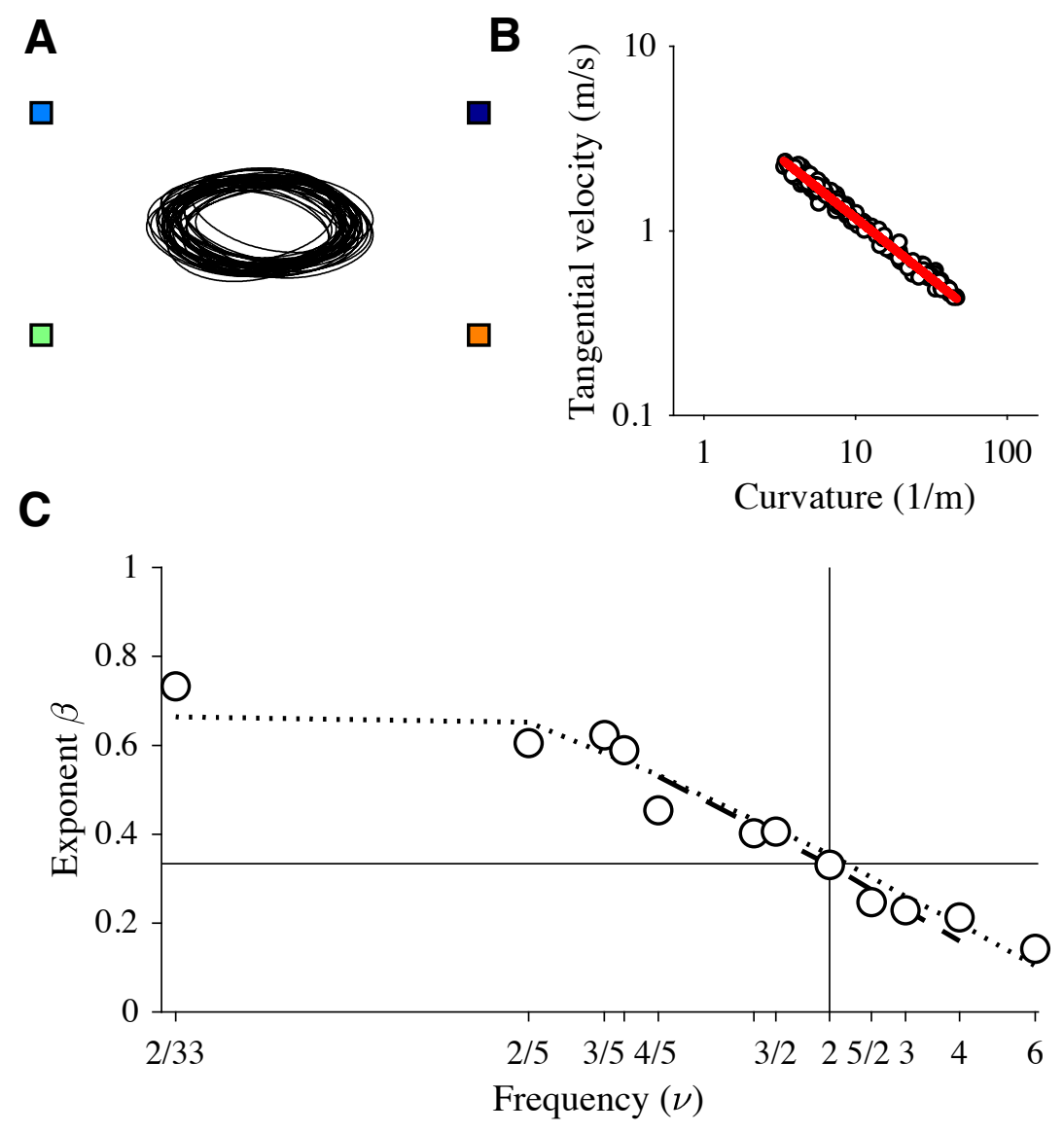

Figure 6 
A

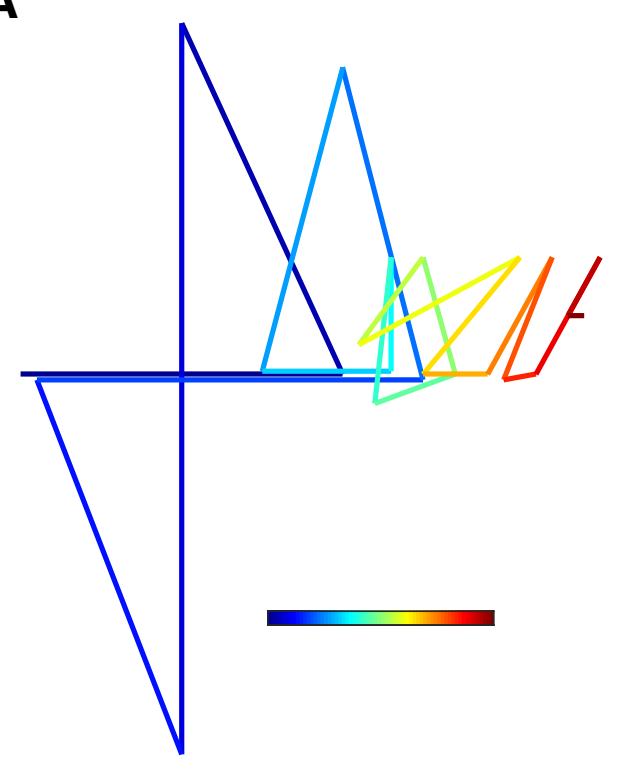

B

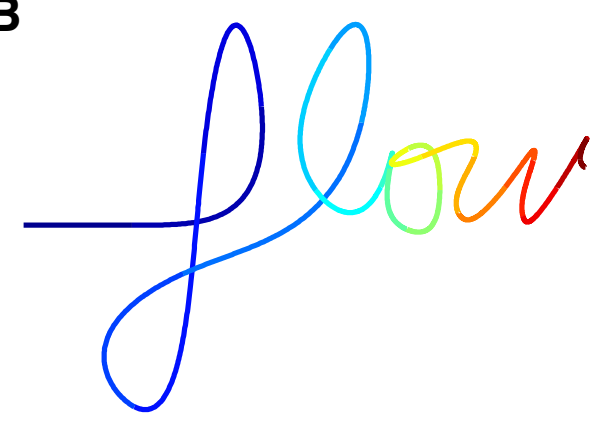

C

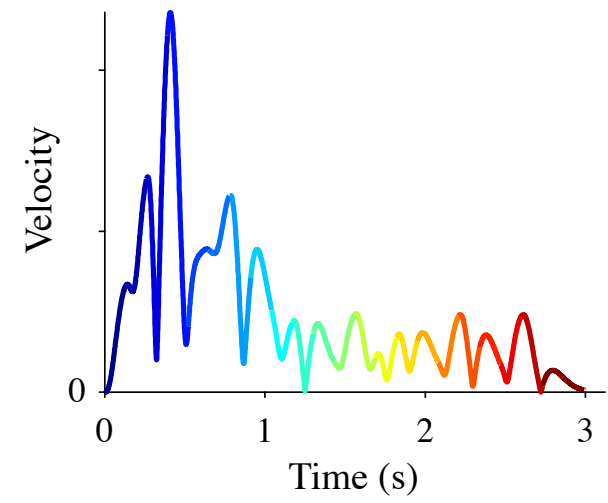

Figure 7 
A
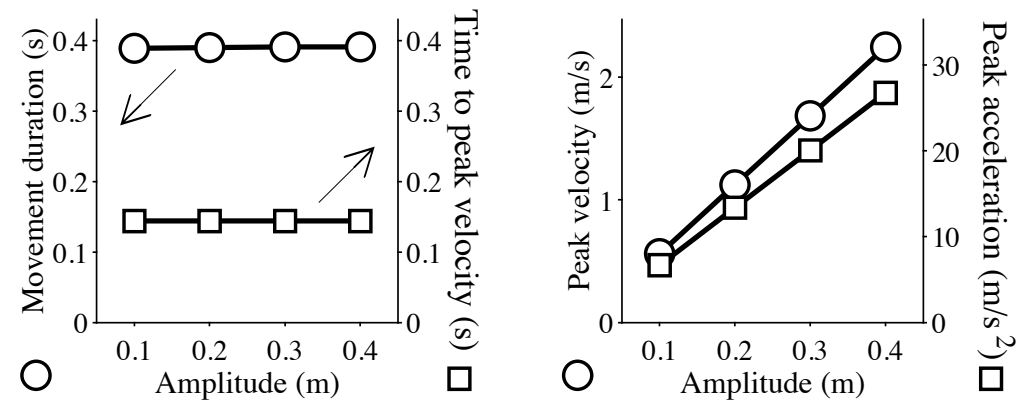

B
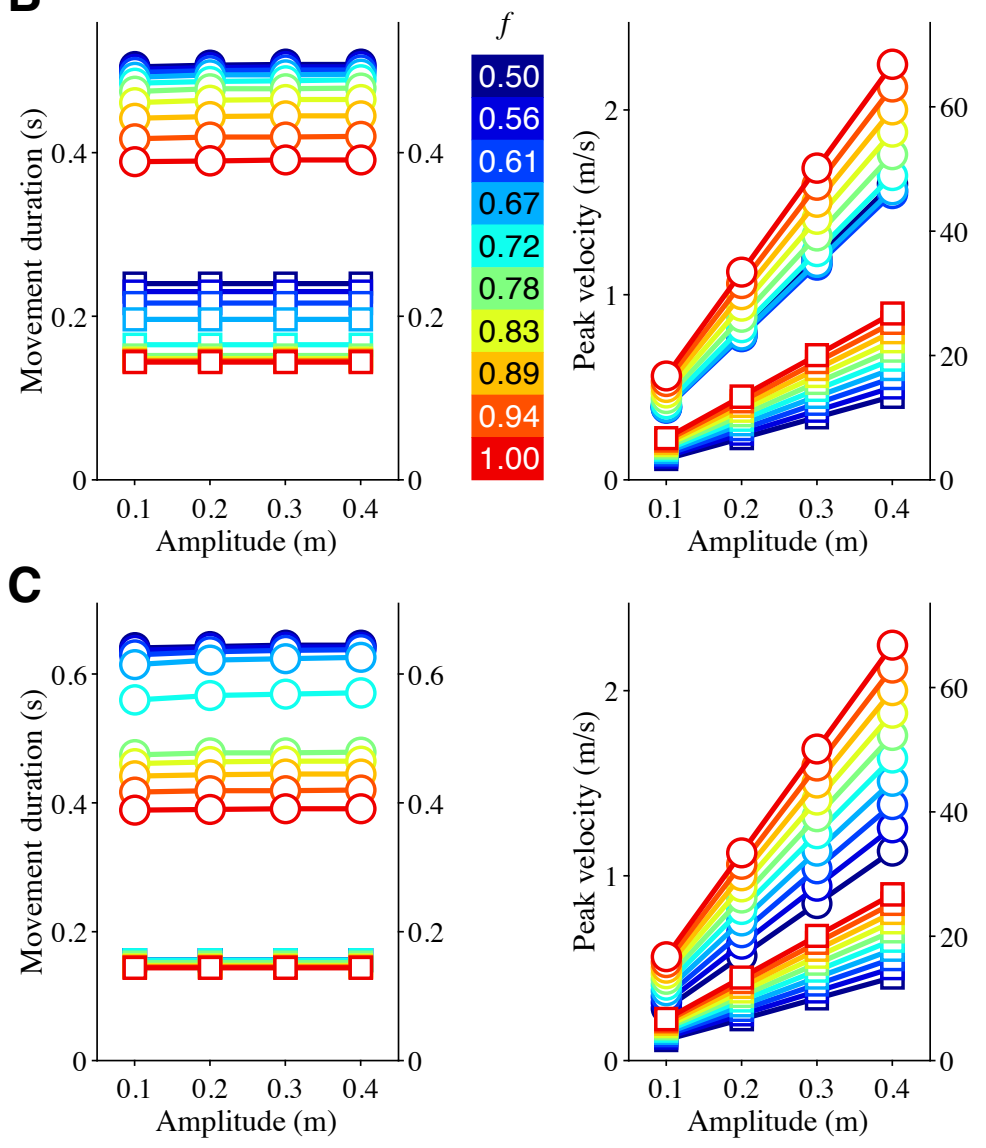

Figure 8 
A

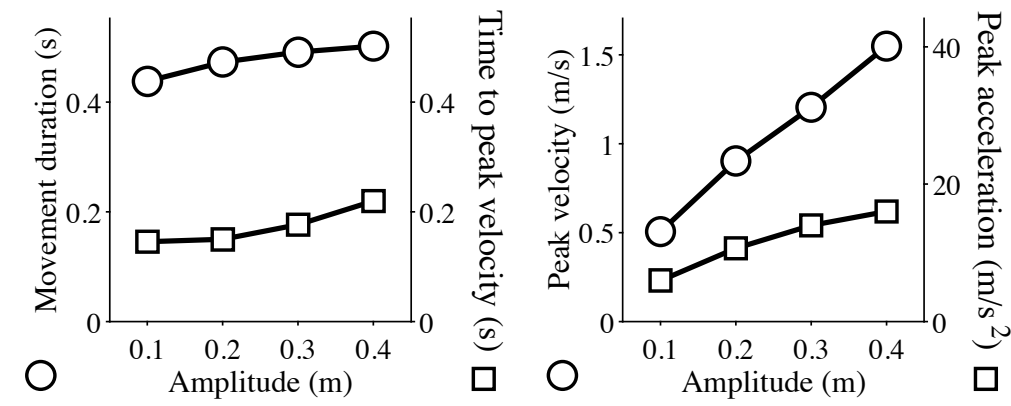

B
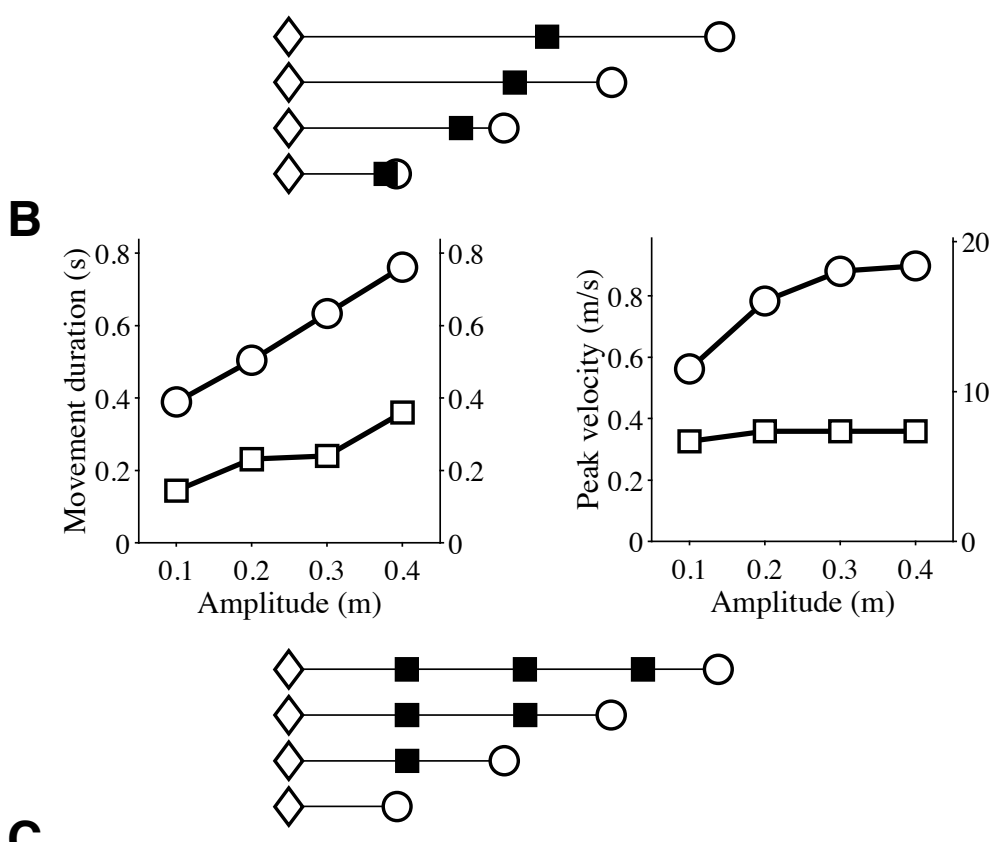

C
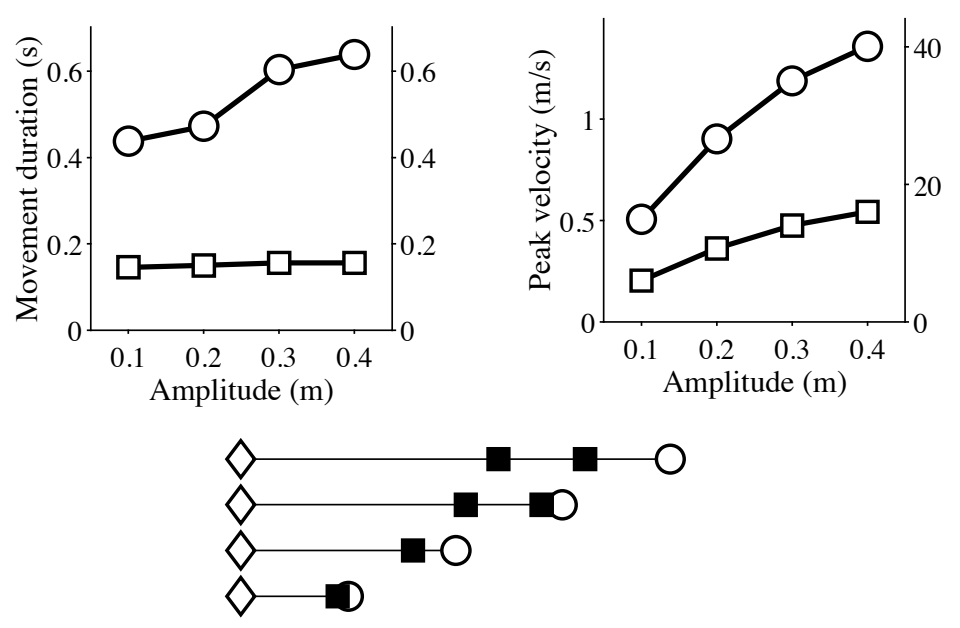

Figure 9 

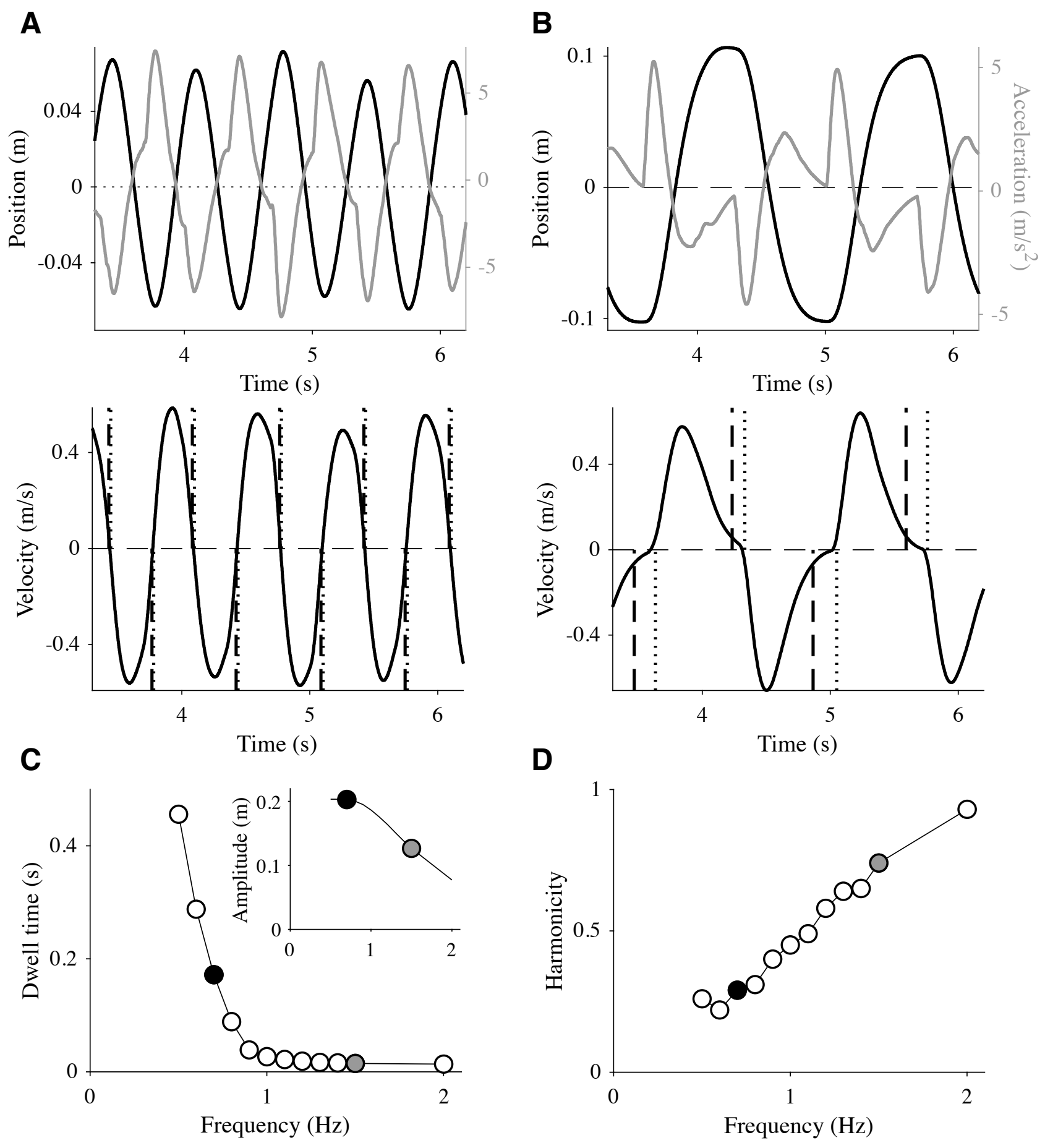

Figure 10 


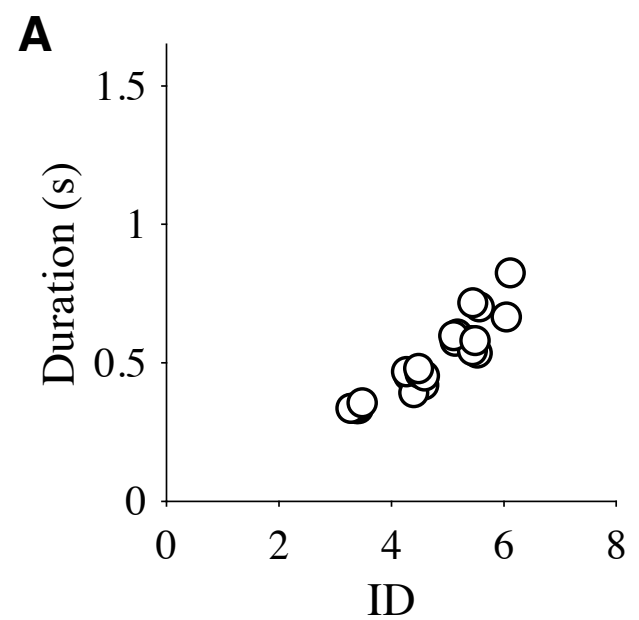

C

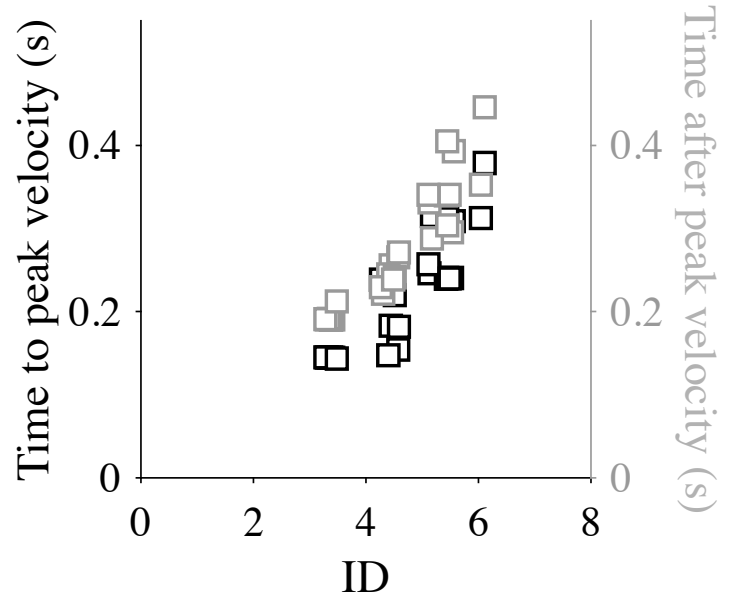

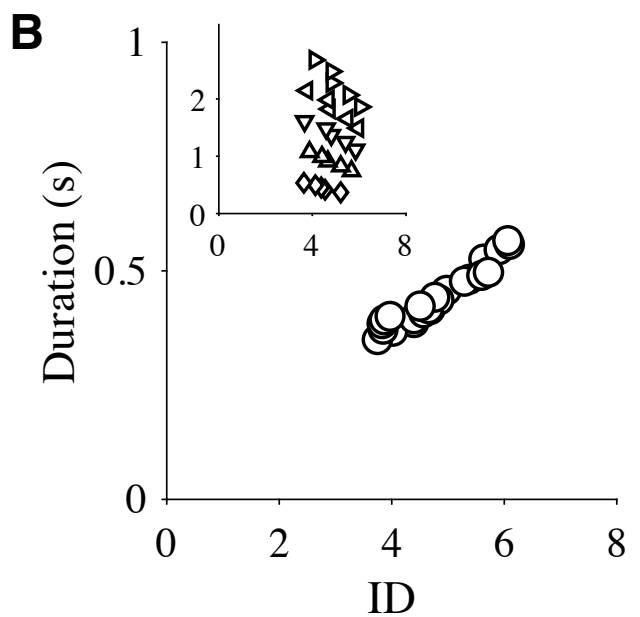

D

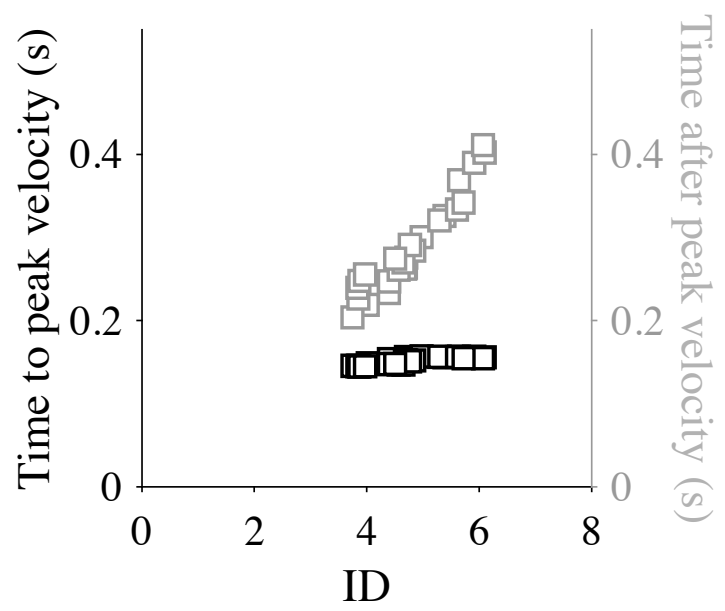

Figure 11 

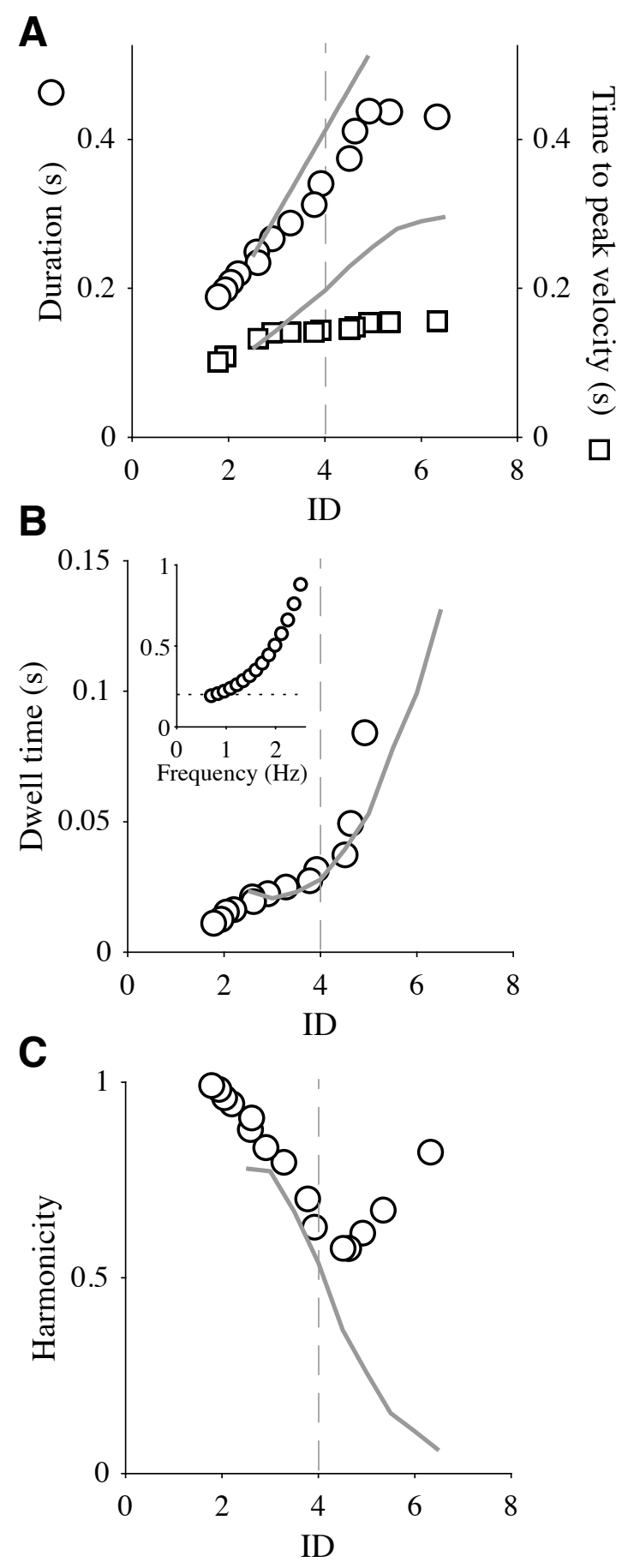

Figure 12 


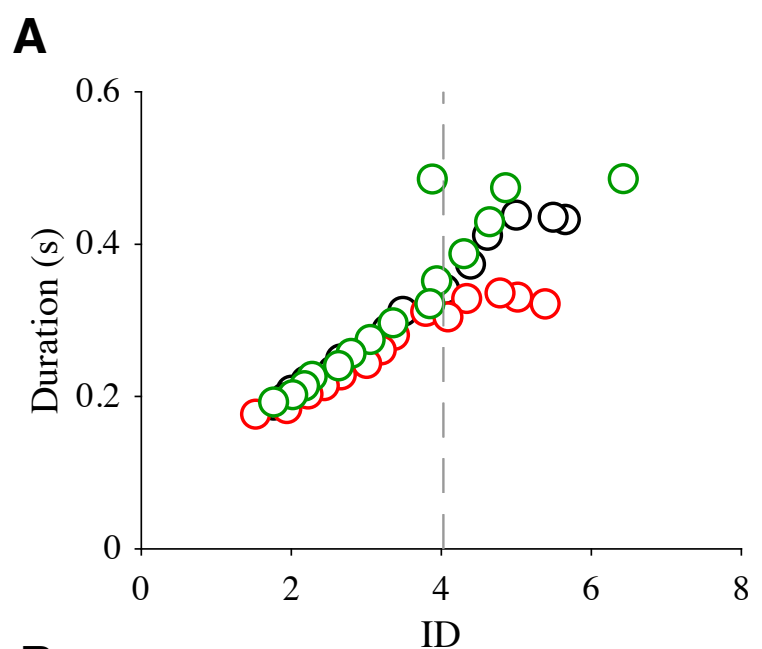

B

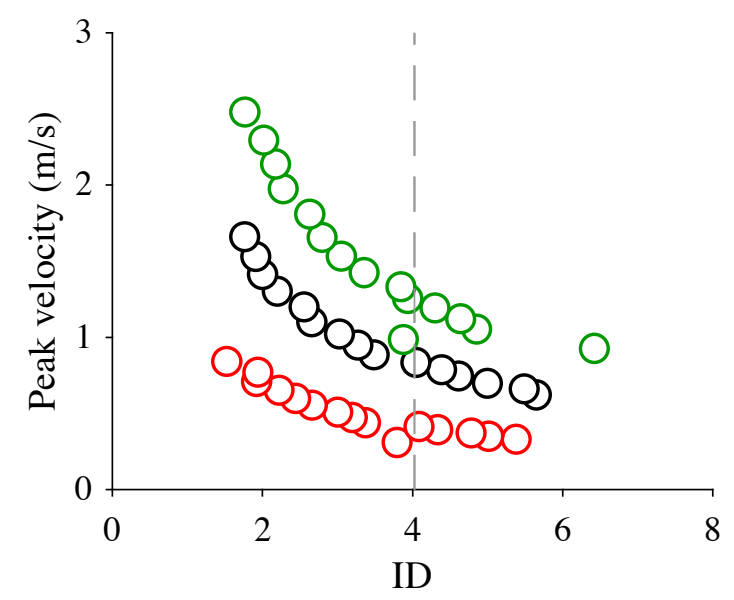

Figure 13 

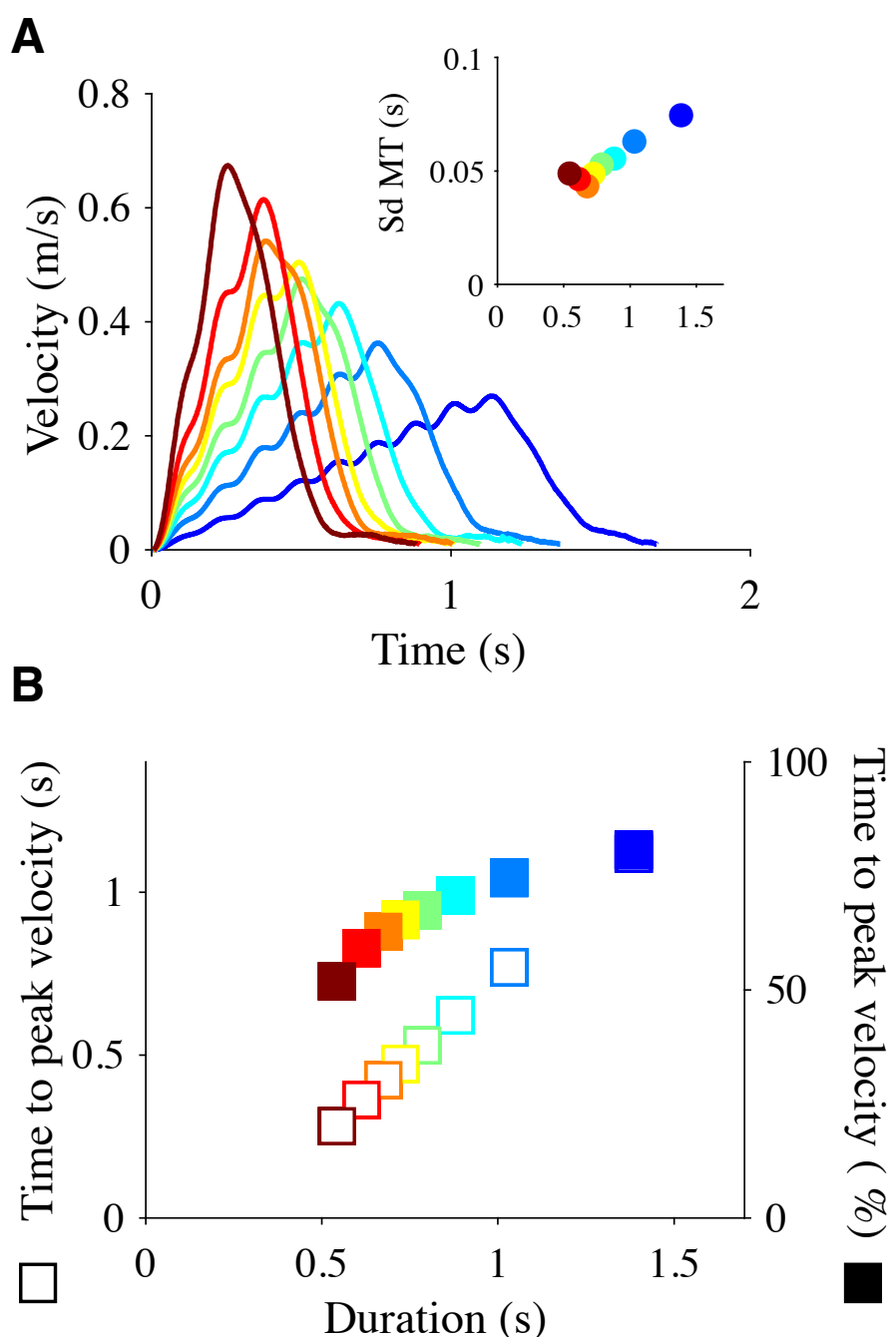

Figure 14 
A
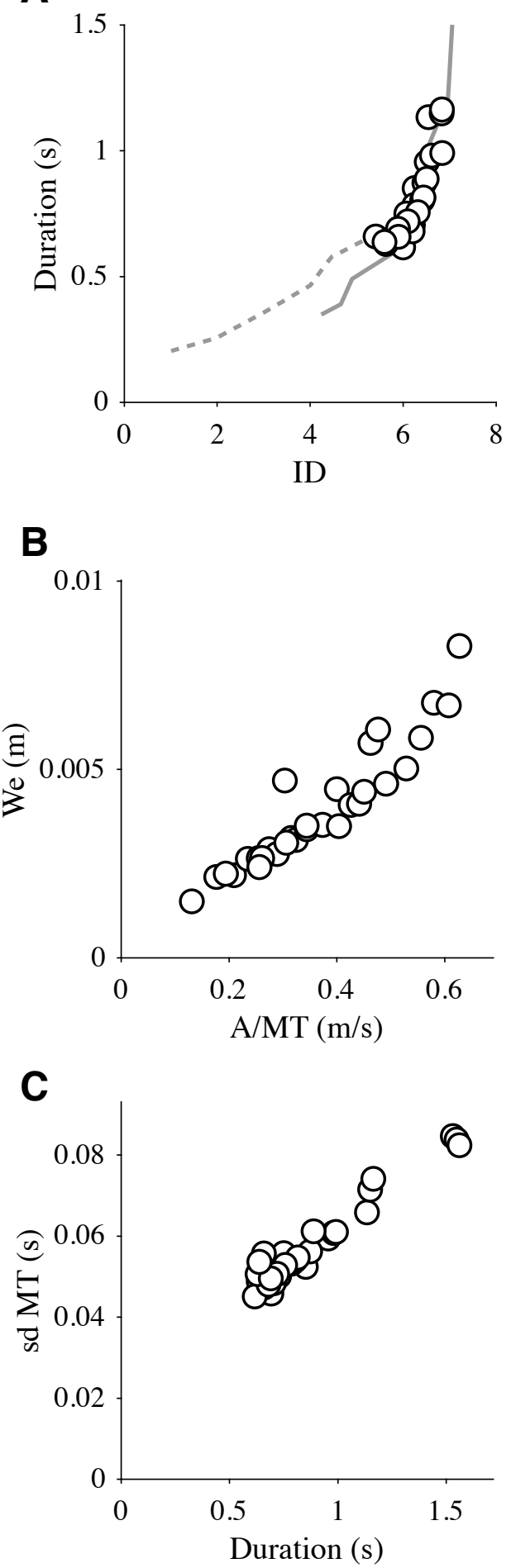

Figure 15 


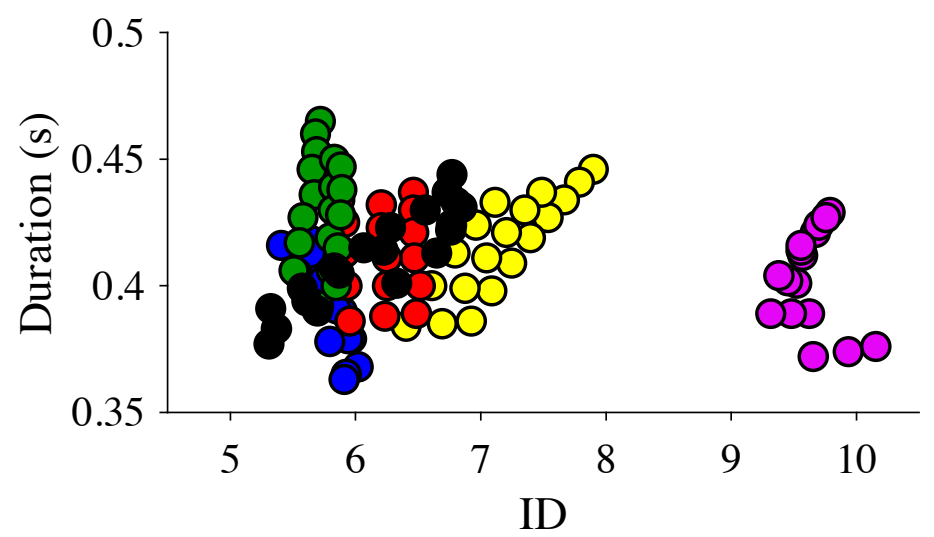

Figure 16 

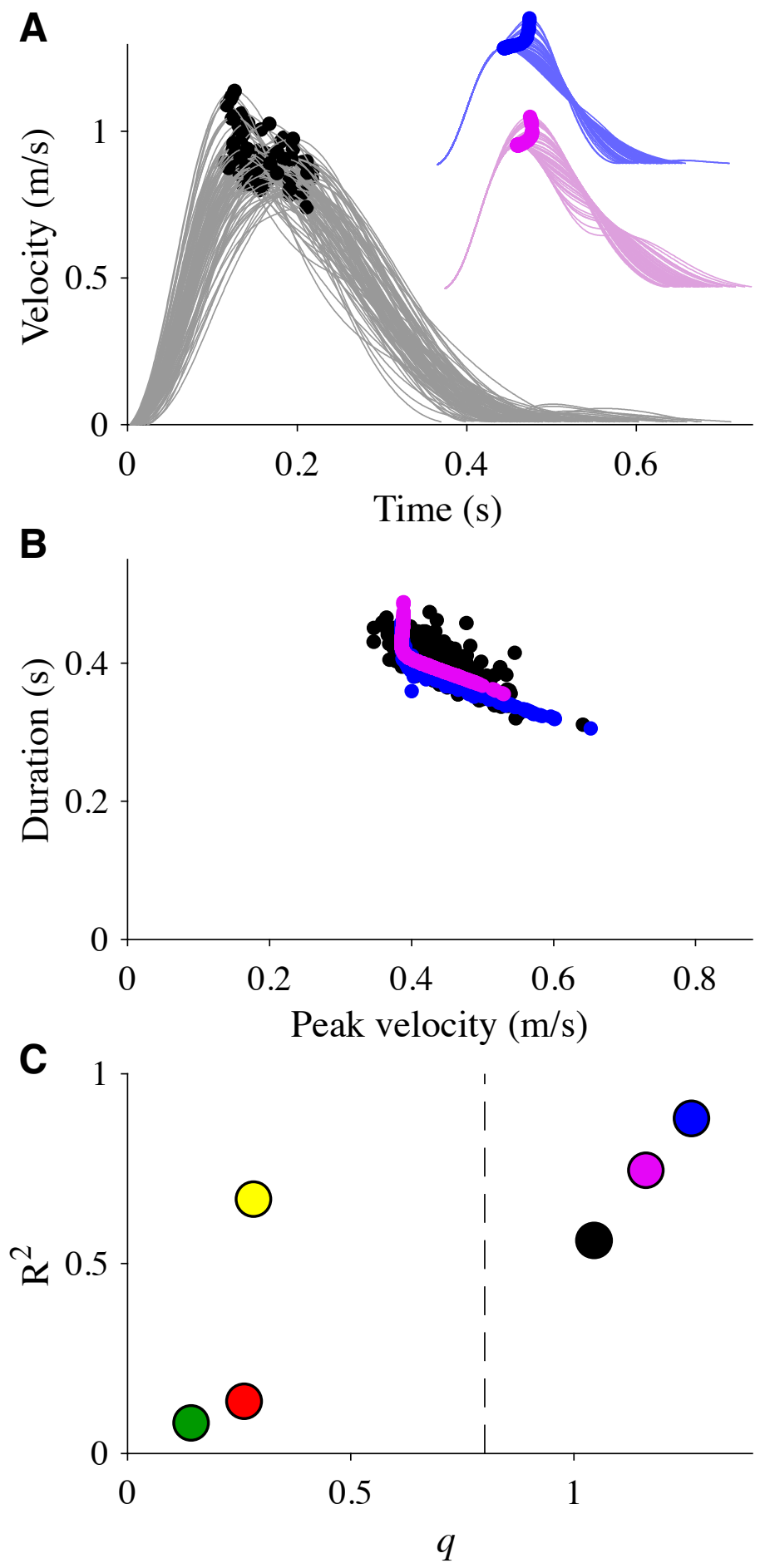

Figure 17 

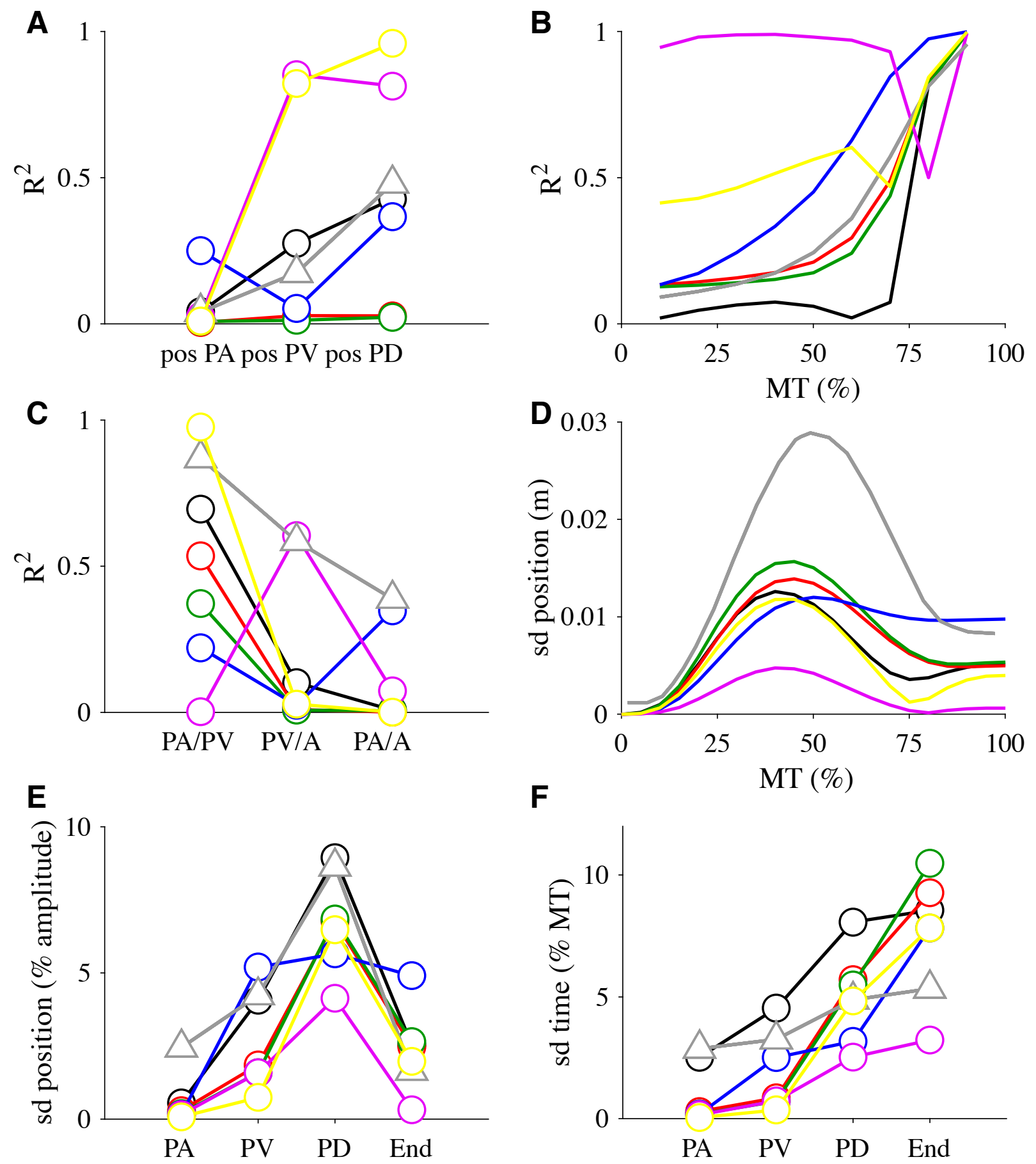

F

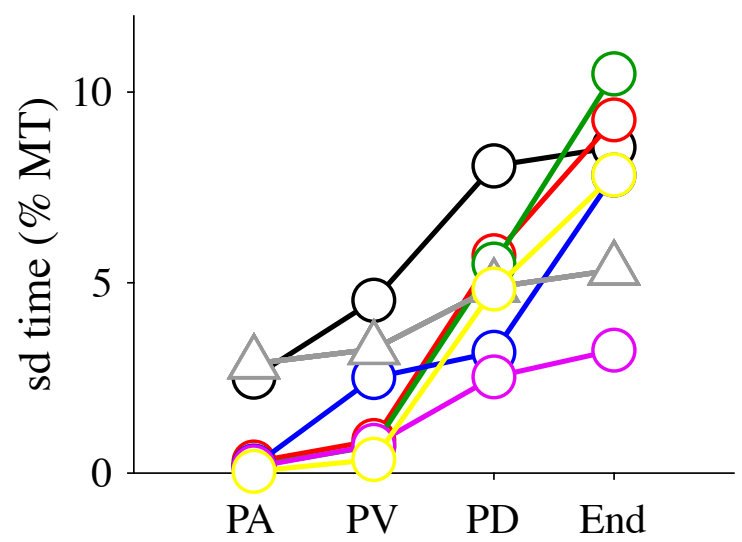

Figure 18 

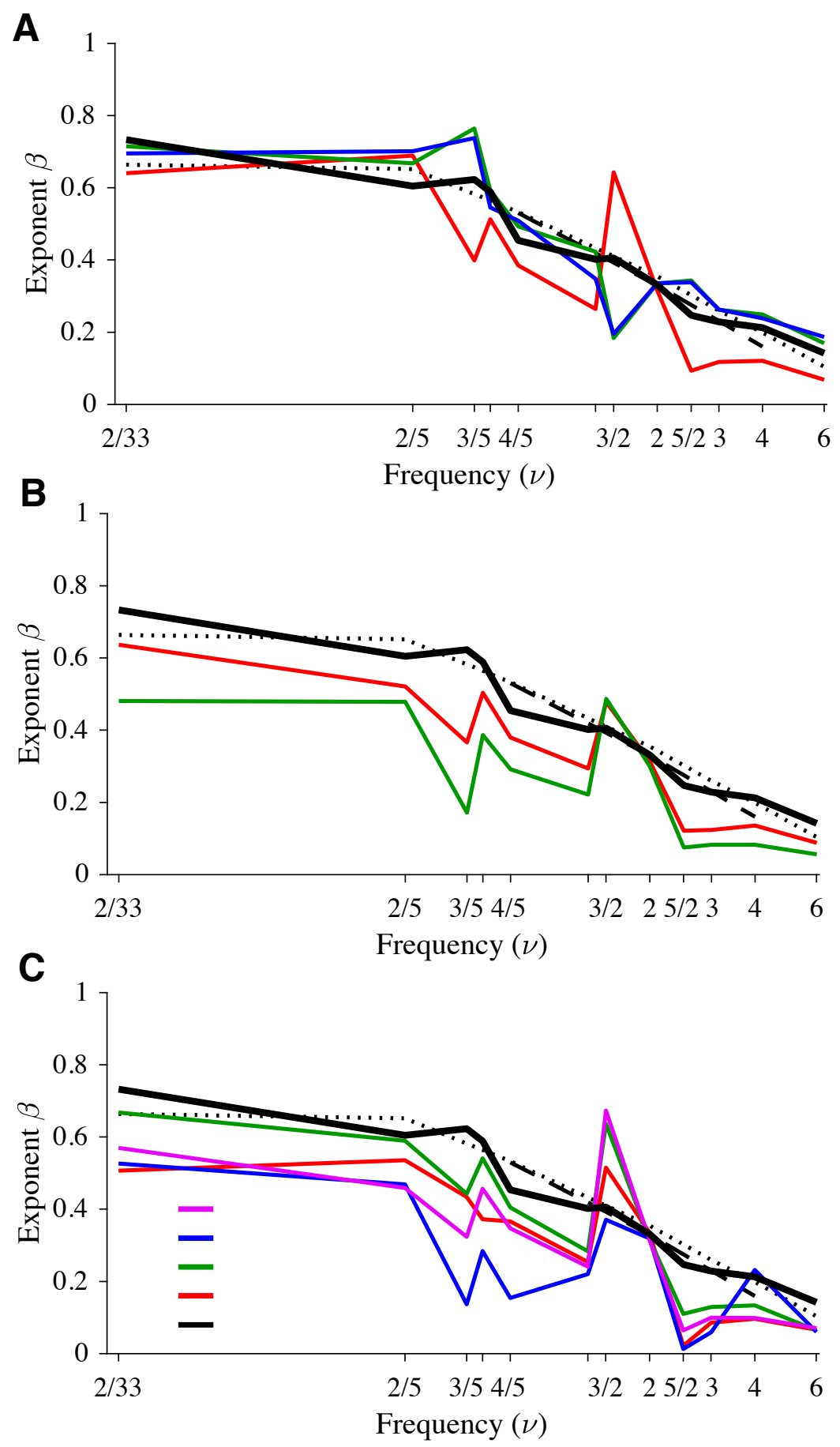

Figure A1 
A

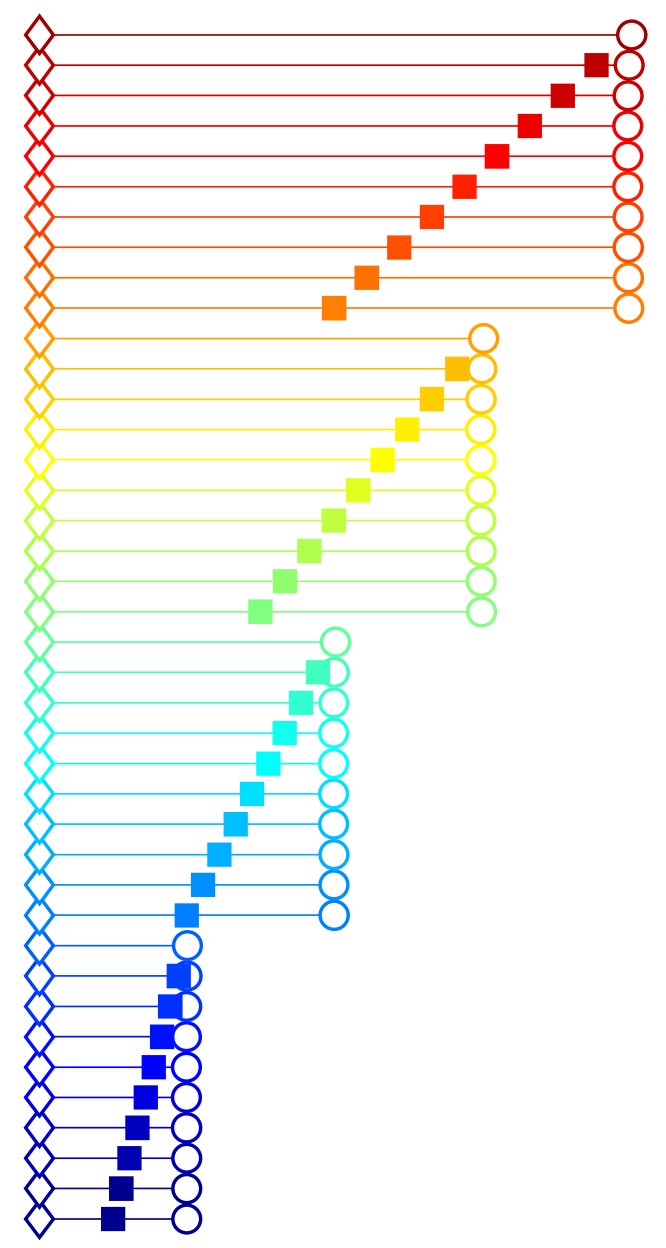

B

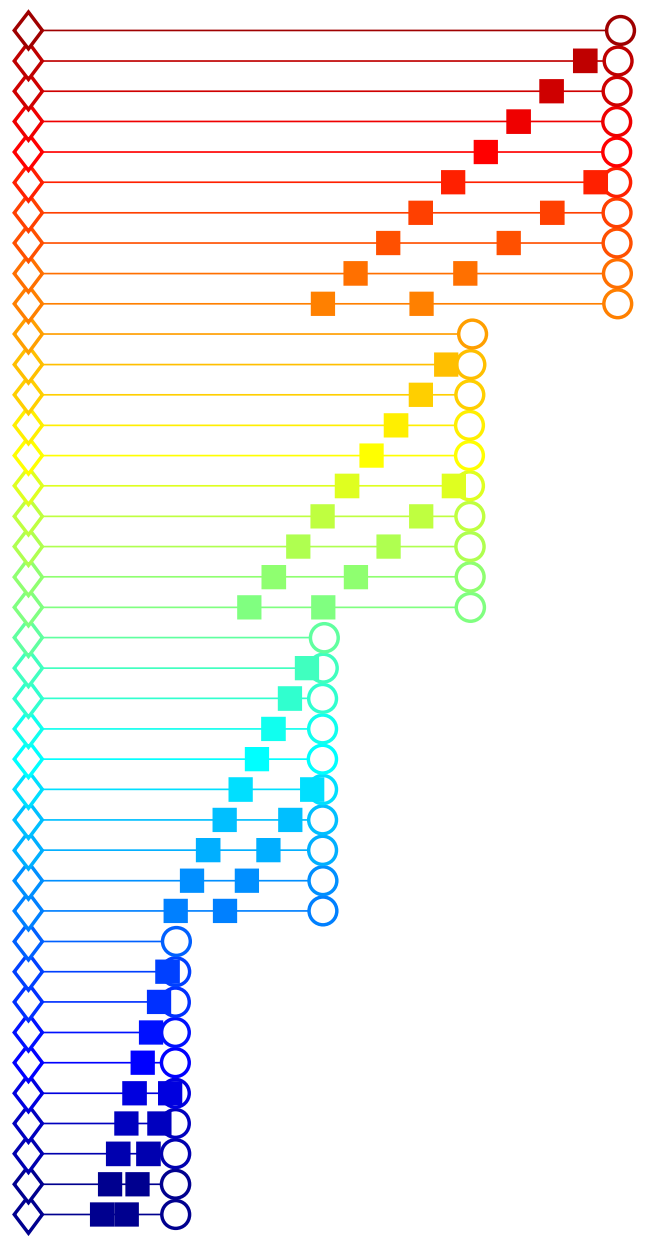

Figure B1 
A
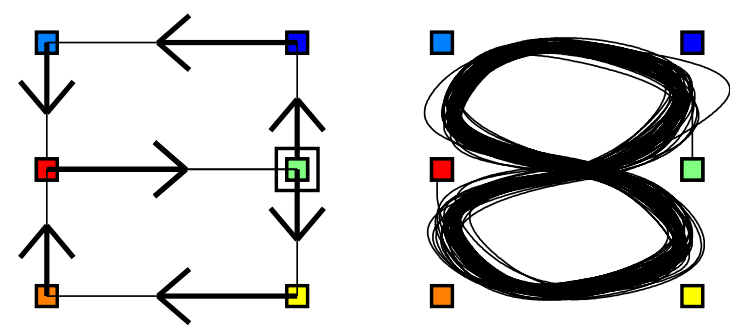

8 points

B

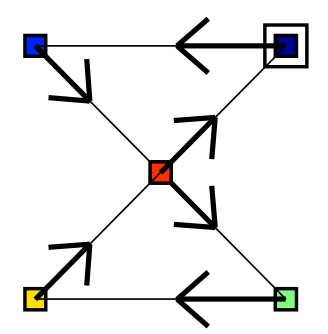

$0.95 \mathrm{~Hz}$

C
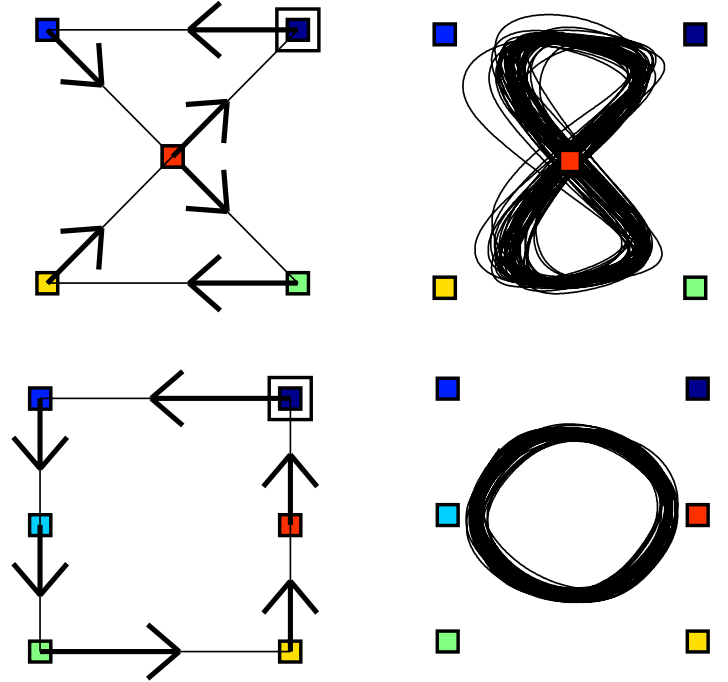

6 points

1.27 Hz

D
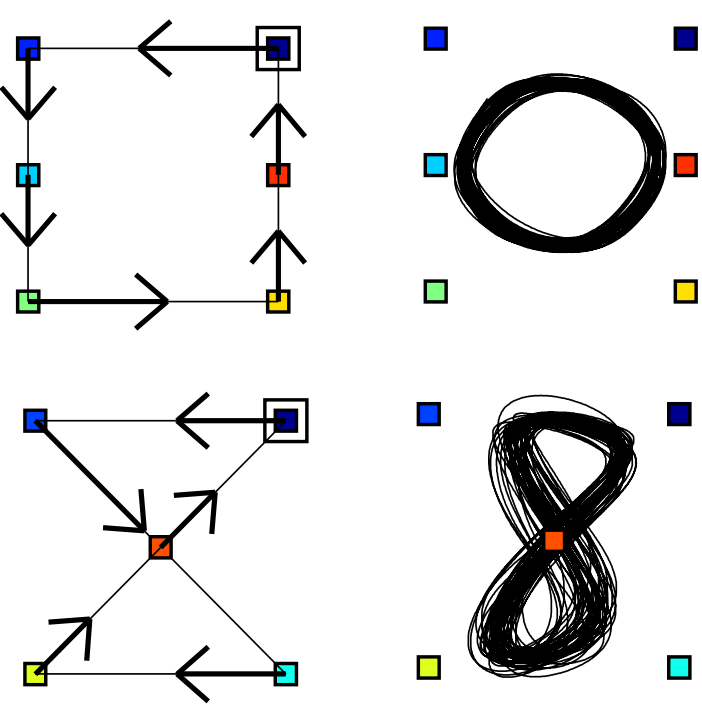

6 points

$1.28 \mathrm{~Hz}$

E
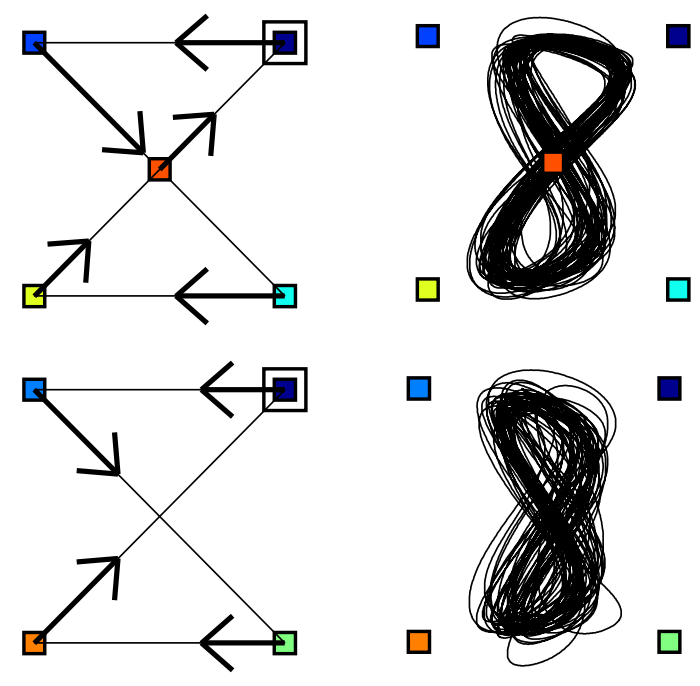

5 points

$1.52 \mathrm{~Hz}$

F
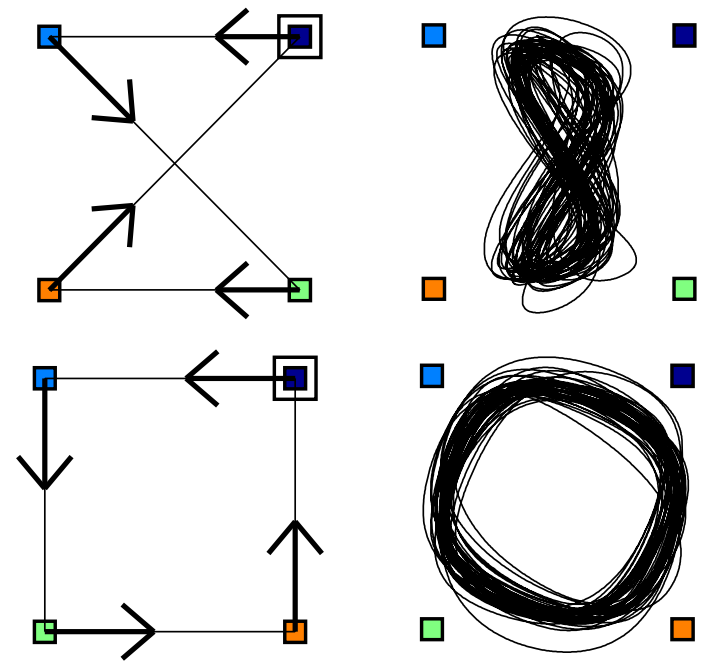

4 points

$1.89 \mathrm{~Hz}$

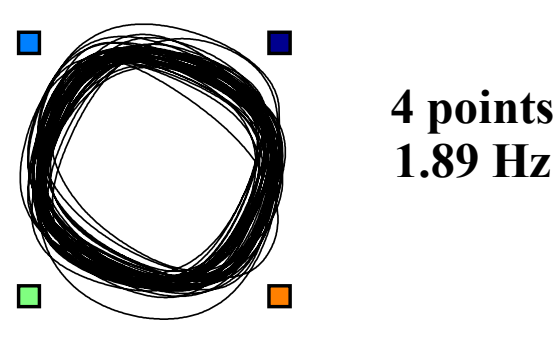

Figure $\mathrm{C} 1$ 


\section{Online Supplemental Material}

\section{A computational theory for the production of limb movements \\ Emmanuel Guigon}

March 12, 2020

Here, we present the mathematical background necessary to understand the proposed model.

\section{Optimal control with terminal constraints}

We consider a dynamical system

$$
\dot{\boldsymbol{x}}(t)=f[\boldsymbol{x}(t), \boldsymbol{u}(t)]
$$

where $\boldsymbol{x} \in \mathbb{R}^{n}$ is the state of the system and $\boldsymbol{u} \in \mathbb{R}^{m}$ a control vector. An optimal control problem for this system is to find the control vector $\boldsymbol{u}(t)$ for $t \in\left[t_{0} ; t_{f}\right]$ to minimize a performance index

$$
J=\int_{t_{0}}^{t_{f}} L[\boldsymbol{x}(t), \boldsymbol{u}(t)] d t
$$

with boundary conditions

$$
\boldsymbol{x}\left(t_{0}\right)=\boldsymbol{x}_{0} \quad \psi\left[\boldsymbol{x}\left(t_{f}\right)\right]=0 .
$$

This problem is the generic formulation corresponding to Equations 1,2,3 of the article. 


\section{Mayer formulation}

We first show that the optimal control problem defined by Eq. S1, Eq. S2 and Eq. S3 can be equivalently written

$$
\begin{gathered}
\dot{\tilde{\boldsymbol{x}}}(t)=\tilde{f}[\tilde{\boldsymbol{x}}(t), \boldsymbol{u}(t)] \\
\tilde{J}=\phi\left[\tilde{\boldsymbol{x}}\left(t_{f}\right)\right] \\
\tilde{\boldsymbol{x}}\left(t_{0}\right)=\tilde{\boldsymbol{x}}_{0} \quad \tilde{\psi}\left[\tilde{\boldsymbol{x}}\left(t_{f}\right)\right]=0
\end{gathered}
$$

which is called the Mayer formulation and which is simpler for numerical methods.

We consider the supplementary state variable $z$ defined by

$$
\dot{z}(t)=L[\boldsymbol{x}(t), \boldsymbol{u}(t)]
$$

and $z\left(t_{0}\right)=0$. Thus $J=z\left(t_{f}\right)$. We define the new state variable

$$
\tilde{\boldsymbol{x}}(t)=\left(\begin{array}{c}
z(t) \\
\boldsymbol{x}(t)
\end{array}\right) .
$$

We can reformulate the optimal control problem in the following way: find the control vector $\boldsymbol{u}(t)$ to minimize

$$
\tilde{J}=\phi\left[\tilde{\boldsymbol{x}}\left(t_{f}\right)\right]=z\left(t_{f}\right)
$$

subject to

$$
\dot{\tilde{\boldsymbol{x}}}(t)=\tilde{f}[\tilde{\boldsymbol{x}}(t), \boldsymbol{u}(t)]=\left(\begin{array}{c}
L[\boldsymbol{x}(t), \boldsymbol{u}(t)] \\
f[\boldsymbol{x}(t), \boldsymbol{u}(t)]
\end{array}\right)
$$

and

$$
\tilde{\boldsymbol{x}}\left(t_{0}\right)=\tilde{\boldsymbol{x}}_{0}=\left(\begin{array}{l}
0 \\
\boldsymbol{x}_{0}
\end{array}\right) \quad \tilde{\psi}\left[\tilde{\boldsymbol{x}}\left(t_{f}\right)\right]=\left(\begin{array}{l}
0 \\
\psi\left[\boldsymbol{x}\left(t_{f}\right)\right]
\end{array}\right)=0 .
$$

Thus we can remove the integral term in the performance index. In the following we consider the problem defined by Eq. S4, Eq. S5 and Eq. S6. For simplicity, we remove the tilde sign.

\section{Solution}

We adjoin the constraints to the performance index with Lagrange multipliers $\nu$ and $\boldsymbol{\lambda}(t)$

$$
\bar{J}=\phi+\boldsymbol{\nu}^{T} \psi+\int_{t_{0}}^{t_{f}} \boldsymbol{\lambda}^{T}(t)\{f[\boldsymbol{x}(t), \boldsymbol{u}(t)]-\dot{\boldsymbol{x}}(t)\} d t .
$$


The Hamiltonian function is

$$
\mathcal{H}[\boldsymbol{x}(t), \boldsymbol{u}(t), \boldsymbol{\lambda}(t)]=\mathcal{H}(t)=\boldsymbol{\lambda}^{T}(t) f[\boldsymbol{x}(t), \boldsymbol{u}(t)] .
$$

The generalized performance index can be written

$$
\bar{J}=\Phi\left[\boldsymbol{x}\left(t_{f}\right)\right]-\boldsymbol{\lambda}^{T}\left(t_{f}\right) \boldsymbol{x}\left(t_{f}\right)+\boldsymbol{\lambda}^{T}\left(t_{0}\right) \boldsymbol{x}\left(t_{0}\right)+\int_{t_{0}}^{t_{f}}\left\{\mathcal{H}(t)+\dot{\boldsymbol{\lambda}}^{T}(t) \boldsymbol{x}(t)\right\} d t
$$

following integration of the $\boldsymbol{\lambda}^{T} \dot{\boldsymbol{x}}$ by parts, where

$$
\Phi=\phi+\nu^{T} \psi
$$

A variation of $\bar{J}$ writes

$$
\delta \bar{J}=\left[\left(\Phi_{x}-\boldsymbol{\lambda}^{T}\right) \delta x\right]_{t=t_{f}}+\left[\lambda^{T} \delta x\right]_{t=t_{0}}+\int_{t_{0}}^{t_{f}}\left[\left(\mathcal{H}_{x}+\dot{\boldsymbol{\lambda}}^{T}\right) \delta x+\mathcal{H}_{u} \delta u\right] d t
$$

for variations $\delta x(t)$ and $\delta u(t)$. The Lagrange mutlipliers are chosen so that the coefficients of $\delta x(t)$ and $\delta x\left(t_{f}\right)$ vanish

$$
\dot{\boldsymbol{\lambda}}^{T}=-\mathcal{H}_{x}=-\boldsymbol{\lambda}^{T} f_{x},
$$

with boundary conditions

$$
\boldsymbol{\lambda}^{T}\left(t_{f}\right)=\phi_{x}\left(t_{f}\right)+\boldsymbol{\nu}^{T} \psi_{x}\left(t_{f}\right) .
$$

For a stationary solution, $\delta \bar{J}=0$ for arbitrary $\delta u(t)$, which implies

$$
\mathcal{H}_{u}=\lambda^{T} f_{u}=0 \quad t_{0} \leq t \leq t_{f} .
$$

The problem defined by Eq. S1, Eq. S12, Eq. S13 and Eq. S14 is a two-point boundary value problem which can be solved by classical integration methods (Bryson 1999).

\section{Linear case}

In the linear case, the problem is a first-order linear dynamical system which can be solved explicitly. The solution consists in a $2 n \times 2 n$ matrix $\mathbf{D}(t)$ such that

$$
\left(\begin{array}{l}
\boldsymbol{x}(t) \\
\boldsymbol{\lambda}(t)
\end{array}\right)=\mathbf{D}(t) \mathcal{C}
$$


is the solution at time $t$, where $\mathcal{C} \in \mathbb{R}^{2 n}$ is a vector determined by the boundary conditions (Eq. S3). To simplify we use $\psi\left[\boldsymbol{x}\left(t_{f}\right)\right]=\boldsymbol{x}\left(t_{f}\right)-\boldsymbol{x}_{f}$, but more complex boundary conditions can be handled as well (see below). To obtain $\mathcal{C}$, we write

$$
\left(\begin{array}{l}
\boldsymbol{x}_{0} \\
\boldsymbol{\lambda}\left(t_{0}\right)
\end{array}\right)=\mathbf{D}\left(t_{0}\right) \mathcal{C}=\left(\begin{array}{ll}
\mathbf{D}_{11}\left(t_{0}\right) & \mathbf{D}_{12}\left(t_{0}\right) \\
\mathbf{D}_{21}\left(t_{0}\right) & \mathbf{D}_{22}\left(t_{0}\right)
\end{array}\right)\left(\begin{array}{l}
\mathcal{C}_{1} \\
\mathcal{C}_{2}
\end{array}\right)
$$

and

$$
\left(\begin{array}{l}
\boldsymbol{x}_{f} \\
\boldsymbol{\lambda}\left(t_{f}\right)
\end{array}\right)=\mathbf{D}\left(t_{f}\right) \mathcal{C}=\left(\begin{array}{ll}
\mathbf{D}_{11}\left(t_{f}\right) & \mathbf{D}_{12}\left(t_{f}\right) \\
\mathbf{D}_{21}\left(t_{f}\right) & \mathbf{D}_{22}\left(t_{f}\right)
\end{array}\right)\left(\begin{array}{l}
\mathcal{C}_{1} \\
\mathcal{C}_{2}
\end{array}\right)
$$

Thus

$$
\left(\begin{array}{ll}
\mathbf{D}_{11}\left(t_{0}\right) & \mathbf{D}_{12}\left(t_{0}\right) \\
\mathbf{D}_{11}\left(t_{f}\right) & \mathbf{D}_{12}\left(t_{f}\right)
\end{array}\right)\left(\begin{array}{l}
\mathcal{C}_{1} \\
\mathcal{C}_{2}
\end{array}\right)=\left(\begin{array}{l}
\boldsymbol{x}_{0} \\
\boldsymbol{x}_{f}
\end{array}\right)
$$

which gives

$$
\mathcal{C}=\left(\begin{array}{ll}
\mathbf{D}_{11}\left(t_{0}\right) & \mathbf{D}_{12}\left(t_{0}\right) \\
\mathbf{D}_{11}\left(t_{f}\right) & \mathbf{D}_{12}\left(t_{f}\right)
\end{array}\right)^{-1}\left(\begin{array}{l}
\boldsymbol{x}_{0} \\
\boldsymbol{x}_{f}
\end{array}\right)
$$

\section{Complete treatement of a linear case}

Here we consider the problem of controlling an inertial point actuated by a linear muscle with a quadratic cost function. In Mayer formulation, the problem can be written

$$
\left\{\begin{array}{l}
\dot{x}_{1}=u^{2} / 2 \\
\dot{x}_{2}=x_{3} \\
\dot{x}_{3}=x_{4} / m \\
\dot{x}_{4}=\left(-x_{4}+x_{5}\right) / \tau \\
\dot{x}_{5}=\left(-x_{5}+u\right) / \tau
\end{array}\right.
$$

The Hamiltonian (Eq. S10) writes

$$
\begin{aligned}
\mathcal{H}= & \lambda_{1} u^{2} / 2+\lambda_{2} x_{3}+\lambda_{3} x_{4} / m+ \\
& \lambda_{4}\left(-x_{4}+x_{5}\right) / \tau+\lambda_{5}\left(-x_{5}+u\right) / \tau
\end{aligned}
$$

from which the adjoint system (Eq. S12) can be obtained 


$$
\begin{aligned}
& \dot{\lambda}_{1}=-\frac{\partial \mathcal{H}}{\partial x_{1}}=0 \\
& \dot{\lambda}_{2}=-\frac{\partial \mathcal{H}}{\partial x_{2}}=0 \\
& \dot{\lambda}_{3}=-\frac{\partial \mathcal{H}}{\partial x_{3}}=-\lambda_{2} \\
& \dot{\lambda}_{4}=-\frac{\partial \mathcal{H}}{\partial x_{4}}=-\lambda_{3} / m+\lambda_{4} / \tau \\
& \dot{\lambda}_{5}=-\frac{\partial \mathcal{H}}{\partial x_{5}}=-\lambda_{4} / \tau+\lambda_{5} / \tau
\end{aligned}
$$

The transversal condition (Eq. S14) is

$$
\mathcal{H}_{u}=\lambda_{1} u+\lambda_{5} / \tau=0,
$$

where $\lambda_{1}$ is a constant set at 1 . The corresponding boundary value problem is

$$
\left\{\begin{array}{l}
\dot{x}_{2}=x_{3} \\
\dot{x}_{3}=x_{4} / m \\
\dot{x}_{4}=\left(-x_{4}+x_{5}\right) / \tau \\
\dot{x}_{5}=\left(-x_{5}-\lambda_{5} / \tau\right) / \tau \\
\dot{\lambda}_{2}=0 \\
\dot{\lambda}_{3}=-\lambda_{2} \\
\dot{\lambda}_{4}=-\lambda_{3} / m+\lambda_{4} / \tau \\
\dot{\lambda}_{5}=-\lambda_{4} / \tau+\lambda_{5} / \tau
\end{array}\right.
$$

The constraints are defined by function $\Phi$ (Eq. S11) which can take different forms:

- Full constraints: position, velocity, activation, excitation

$$
\begin{aligned}
\Phi= & x_{1}\left(t_{f}\right)+\nu_{2}\left[x_{2}\left(t_{f}\right)-x_{2}^{f}\right]+\nu_{3}\left[x_{3}\left(t_{f}\right)-x_{3}^{f}\right]+ \\
& \nu_{4}\left[x_{4}\left(t_{f}\right)-x_{4}^{f}\right]+\nu_{5}\left[x_{5}\left(t_{f}\right)-x_{5}^{f}\right]
\end{aligned}
$$

- Partial constraints: position, velocity, activation

$$
\begin{aligned}
\Phi= & x_{1}\left(t_{f}\right)+\nu_{2}\left[x_{2}\left(t_{f}\right)-x_{2}^{f}\right]+\nu_{3}\left[x_{3}\left(t_{f}\right)-x_{3}^{f}\right]+ \\
& \nu_{4}\left[x_{4}\left(t_{f}\right)-x_{4}^{f}\right]
\end{aligned}
$$


- Partial constraints: position, velocity

$$
\Phi=x_{1}\left(t_{f}\right)+\nu_{2}\left[x_{2}\left(t_{f}\right)-x_{2}^{f}\right]+\nu_{3}\left[x_{3}\left(t_{f}\right)-x_{3}^{f}\right]
$$

- Partial constraints: position

$$
\Phi=x_{1}\left(t_{f}\right)+\nu_{2}\left[x_{2}\left(t_{f}\right)-x_{2}^{f}\right]
$$

The initial boundary conditions are

$$
x_{2}\left(t_{0}\right)=x_{2}^{0} \quad x_{3}\left(t_{0}\right)=x_{3}^{0} \quad x_{4}\left(t_{0}\right)=x_{4}^{0} \quad x_{5}\left(t_{0}\right)=x_{5}^{0}
$$

The final boundary conditions are obtained using Eq. S13:

- Full constraints: position, velocity, activation, excitation

$$
\begin{gathered}
x_{2}\left(t_{f}\right)=x_{2}^{f} \quad x_{3}\left(t_{f}\right)=x_{3}^{f} \quad x_{4}\left(t_{f}\right)=x_{4}^{f} \quad x_{5}\left(t_{f}\right)=x_{5}^{f} \\
\lambda_{1}\left(t_{f}\right)=1 \quad \lambda_{2}\left(t_{f}\right)=\nu_{2} \quad \lambda_{3}\left(t_{f}\right)=\nu_{3} \quad \lambda_{4}\left(t_{f}\right)=\nu_{4} \quad \lambda_{5}\left(t_{f}\right)=\nu_{5}
\end{gathered}
$$

- Partial constraints: position, velocity, activation

$$
\begin{gathered}
x_{2}\left(t_{f}\right)=x_{2}^{f} \quad x_{3}\left(t_{f}\right)=x_{3}^{f} \quad x_{4}\left(t_{f}\right)=x_{4}^{f} \\
\lambda_{1}\left(t_{f}\right)=1 \quad \lambda_{2}\left(t_{f}\right)=\nu_{2} \quad \lambda_{3}\left(t_{f}\right)=\nu_{3} \quad \lambda_{4}\left(t_{f}\right)=\nu_{4} \quad \lambda_{5}\left(t_{f}\right)=0
\end{gathered}
$$

- Partial constraints: position, velocity

$$
\begin{gathered}
x_{2}\left(t_{f}\right)=x_{2}^{f} \quad x_{3}\left(t_{f}\right)=x_{3}^{f} \\
\lambda_{1}\left(t_{f}\right)=1 \quad \lambda_{2}\left(t_{f}\right)=\nu_{2} \quad \lambda_{3}\left(t_{f}\right)=\nu_{3} \quad \lambda_{4}\left(t_{f}\right)=0 \quad \lambda_{5}\left(t_{f}\right)=0
\end{gathered}
$$

- Partial constraints: position

$$
\begin{gathered}
x_{2}\left(t_{f}\right)=x_{2}^{f} \\
\lambda_{1}\left(t_{f}\right)=1 \quad \lambda_{2}\left(t_{f}\right)=\nu_{2} \quad \lambda_{3}\left(t_{f}\right)=0 \quad \lambda_{4}\left(t_{f}\right)=0 \quad \lambda_{5}\left(t_{f}\right)=0
\end{gathered}
$$


From Eq. S27, the solution consists in a $2 n \times 2 n$ matrix $\mathbf{D}(t)(n=4)$ such that

$$
\left(\begin{array}{l}
\boldsymbol{x}(t) \\
\boldsymbol{\lambda}(t)
\end{array}\right)=\mathbf{D}(t) \mathcal{C}
$$

is the solution at time $t$, where $\mathcal{C} \in \mathbb{R}^{2 n}$ is a vector determined by the initial and final boundary conditions. Here $\mathbf{D}$ is the solution to the boundary value problem (Eq. S17), which can be obtained explicitly using tools of symbolic calculus.

To obtain $\mathcal{C}$, we write

$$
\left(\begin{array}{l}
\boldsymbol{x}_{0} \\
\boldsymbol{\lambda}\left(t_{0}\right)
\end{array}\right)=\mathbf{D}\left(t_{0}\right) \mathcal{C}=\mathbf{D}^{0} \mathcal{C}
$$

and

$$
\left(\begin{array}{l}
\boldsymbol{x}_{f} \\
\boldsymbol{\lambda}\left(t_{f}\right)
\end{array}\right)=\mathbf{D}\left(t_{f}\right) \mathcal{C}=\mathbf{D}^{f} \mathcal{C}
$$

and we extract what is known from these relationships in the different cases (full constraints: Eq. S19 and Eq. S20; partial constraints on position, velocity, activation: Eq. S21 and Eq. S22; partial constraints on position, velocity: Eq. S23 and Eq. S24; partial constraints on position: Eq. S25 and Eq. S26).

We obtain a relationship

$$
\mathbf{M q}=\boldsymbol{p}
$$

where $\mathbf{M}$ contains elements of $\mathbf{D}^{0}$ and $\mathbf{D}^{f}, \boldsymbol{q}$ the vector $\mathcal{C}$ and some elements of $\nu$, and $\boldsymbol{p}$ the vector $\boldsymbol{x}_{0}$ and some elements of $\boldsymbol{x}_{f}$. Taking $\boldsymbol{q}=\mathbf{M}^{-1} \boldsymbol{p}$ gives the vector $\mathcal{C}$.

For the case of full constraints (position, velocity, activation, excitation), there are 8 unknowns ( 8 in $\mathcal{C}$ ). We get 4 equations for $\boldsymbol{x}_{0}$ (Eq. S18), and 4 equations for $\boldsymbol{x}_{f}$ (Eq. S19), and Eq. S28 becomes

$$
\left(\begin{array}{llllllll}
\mathbf{D}_{11}^{0} & \mathbf{D}_{12}^{0} & \mathbf{D}_{13}^{0} & \mathbf{D}_{14}^{0} & \mathbf{D}_{15}^{0} & \mathbf{D}_{16}^{0} & \mathbf{D}_{17}^{0} & \mathbf{D}_{18}^{0} \\
\mathbf{D}_{21}^{0} & \mathbf{D}_{22}^{0} & \mathbf{D}_{23}^{0} & \mathbf{D}_{24}^{0} & \mathbf{D}_{25}^{0} & \mathbf{D}_{26}^{0} & \mathbf{D}_{27}^{0} & \mathbf{D}_{28}^{0} \\
\mathbf{D}_{31}^{0} & \mathbf{D}_{32}^{0} & \mathbf{D}_{33}^{0} & \mathbf{D}_{34}^{0} & \mathbf{D}_{35}^{0} & \mathbf{D}_{36}^{0} & \mathbf{D}_{37}^{0} & \mathbf{D}_{38}^{0} \\
\mathbf{D}_{41}^{0} & \mathbf{D}_{42}^{0} & \mathbf{D}_{43}^{0} & \mathbf{D}_{44}^{0} & \mathbf{D}_{45}^{0} & \mathbf{D}_{46}^{0} & \mathbf{D}_{47}^{0} & \mathbf{D}_{48}^{0} \\
\mathbf{D}_{11}^{f} & \mathbf{D}_{12}^{f} & \mathbf{D}_{13}^{f} & \mathbf{D}_{14}^{f} & \mathbf{D}_{15}^{f} & \mathbf{D}_{16}^{f} & \mathbf{D}_{17}^{f} & \mathbf{D}_{18}^{f} \\
\mathbf{D}_{21}^{f} & \mathbf{D}_{22}^{f} & \mathbf{D}_{23}^{f} & \mathbf{D}_{24}^{f} & \mathbf{D}_{25}^{f} & \mathbf{D}_{26}^{f} & \mathbf{D}_{27}^{f} & \mathbf{D}_{28}^{f} \\
\mathbf{D}_{31}^{f} & \mathbf{D}_{32}^{f} & \mathbf{D}_{33}^{f} & \mathbf{D}_{34}^{f} & \mathbf{D}_{35}^{f} & \mathbf{D}_{36}^{f} & \mathbf{D}_{37}^{f} & \mathbf{D}_{38}^{f} \\
\mathbf{D}_{41}^{f} & \mathbf{D}_{42}^{f} & \mathbf{D}_{43}^{f} & \mathbf{D}_{44}^{f} & \mathbf{D}_{45}^{f} & \mathbf{D}_{46}^{f} & \mathbf{D}_{47}^{f} & \mathbf{D}_{48}^{f}
\end{array}\right)\left(\begin{array}{c}
\mathcal{C}_{1} \\
\mathcal{C}_{2} \\
\mathcal{C}_{3} \\
\mathcal{C}_{4} \\
\mathcal{C}_{5} \\
\mathcal{C}_{6} \\
\mathcal{C}_{7} \\
\mathcal{C}_{8}
\end{array}\right)=\left(\begin{array}{c}
x_{2}^{0} \\
x_{3}^{0} \\
x_{4}^{0} \\
x_{5}^{0} \\
x_{2}^{f} \\
x_{3}^{f} \\
x_{4}^{f} \\
x_{5}^{f}
\end{array}\right)
$$

For the case of partial constraints on position, velocity, and activation, there are 11 unknowns ( 8 in $\mathcal{C}, \nu_{2}, \nu_{3}, \nu_{4}$ ). We get 4 equations for $\boldsymbol{x}_{0}$ (Eq. S18), 3 equations for $\boldsymbol{x}_{f}$ (Eq. S21), 4 equations for $\boldsymbol{\lambda}\left(t_{f}\right)$ (Eq. S22), and Eq. S28 becomes 


$$
\left(\begin{array}{lllllllllll}
\mathbf{D}_{11}^{0} & \mathbf{D}_{12}^{0} & \mathbf{D}_{13}^{0} & \mathbf{D}_{14}^{0} & \mathbf{D}_{15}^{0} & \mathbf{D}_{16}^{0} & \mathbf{D}_{17}^{0} & \mathbf{D}_{18}^{0} & 0 & 0 & 0 \\
\mathbf{D}_{21}^{0} & \mathbf{D}_{22}^{0} & \mathbf{D}_{23}^{0} & \mathbf{D}_{24}^{0} & \mathbf{D}_{25}^{0} & \mathbf{D}_{26}^{0} & \mathbf{D}_{27}^{0} & \mathbf{D}_{28}^{0} & 0 & 0 & 0 \\
\mathbf{D}_{31}^{0} & \mathbf{D}_{32}^{0} & \mathbf{D}_{33}^{0} & \mathbf{D}_{34}^{0} & \mathbf{D}_{35}^{0} & \mathbf{D}_{36}^{0} & \mathbf{D}_{37}^{0} & \mathbf{D}_{38}^{0} & 0 & 0 & 0 \\
\mathbf{D}_{41}^{0} & \mathbf{D}_{42}^{0} & \mathbf{D}_{43}^{0} & \mathbf{D}_{44}^{0} & \mathbf{D}_{45}^{0} & \mathbf{D}_{46}^{0} & \mathbf{D}_{47}^{0} & \mathbf{D}_{48}^{0} & 0 & 0 & 0 \\
\mathbf{D}_{11}^{f} & \mathbf{D}_{12}^{f} & \mathbf{D}_{13}^{f} & \mathbf{D}_{14}^{f} & \mathbf{D}_{15}^{f} & \mathbf{D}_{16}^{f} & \mathbf{D}_{17}^{f} & \mathbf{D}_{18}^{f} & 0 & 0 & 0 \\
\mathbf{D}_{21}^{f} & \mathbf{D}_{22}^{f} & \mathbf{D}_{23}^{f} & \mathbf{D}_{24}^{f} & \mathbf{D}_{25}^{f} & \mathbf{D}_{26}^{f} & \mathbf{D}_{27}^{f} & \mathbf{D}_{18}^{f} & 0 & 0 & 0 \\
\mathbf{D}_{31}^{f} & \mathbf{D}_{32}^{f} & \mathbf{D}_{33}^{f} & \mathbf{D}_{34}^{f} & \mathbf{D}_{35}^{f} & \mathbf{D}_{36}^{f} & \mathbf{D}_{37}^{f} & \mathbf{D}_{38}^{f} & 0 & 0 & 0 \\
\mathbf{D}_{51}^{f} & \mathbf{D}_{52}^{f} & \mathbf{D}_{53}^{f} & \mathbf{D}_{54}^{f} & \mathbf{D}_{55}^{f} & \mathbf{D}_{56}^{f} & \mathbf{D}_{57}^{f} & \mathbf{D}_{58}^{f} & -1 & 0 & 0 \\
\mathbf{D}_{61}^{f} & \mathbf{D}_{62}^{f} & \mathbf{D}_{63}^{f} & \mathbf{D}_{64}^{f} & \mathbf{D}_{65}^{f} & \mathbf{D}_{66}^{f} & \mathbf{D}_{67}^{f} & \mathbf{D}_{68}^{f} & 0 & -1 & 0 \\
\mathbf{D}_{71}^{f} & \mathbf{D}_{72}^{f} & \mathbf{D}_{73}^{f} & \mathbf{D}_{74}^{f} & \mathbf{D}_{75}^{f} & \mathbf{D}_{76}^{f} & \mathbf{D}_{77}^{f} & \mathbf{D}_{78}^{f} & 0 & 0 & -1 \\
\mathbf{D}_{81}^{f} & \mathbf{D}_{82}^{f} & \mathbf{D}_{83}^{f} & \mathbf{D}_{84}^{f} & \mathbf{D}_{85}^{f} & \mathbf{D}_{86}^{f} & \mathbf{D}_{87}^{f} & \mathbf{D}_{88}^{f} & 0 & 0 & 0
\end{array}\right)\left(\begin{array}{c}
\mathcal{C}_{1} \\
\mathcal{C}_{2} \\
\mathcal{C}_{3} \\
\mathcal{C}_{4} \\
\mathcal{C}_{5} \\
\mathcal{C}_{6} \\
\mathcal{C}_{7} \\
\mathcal{C}_{8} \\
\nu_{2} \\
\nu_{3} \\
\nu_{4}
\end{array}\right)=\left(\begin{array}{c}
x_{2}^{0} \\
x_{3}^{0} \\
x_{4}^{0} \\
x_{5}^{0} \\
x_{2}^{f} \\
x_{3}^{f} \\
x_{4}^{f} \\
0 \\
0 \\
0 \\
0
\end{array}\right)
$$

For the case of partial constraints on position and velocity, there are 10 unknowns ( 8 in $\left.\mathcal{C}, \nu_{2}, \nu_{3}\right)$. We get 4 equations for $\boldsymbol{x}_{0}$ (Eq. S18), 2 equations for $\boldsymbol{x}_{f}$ (Eq. S23), 4 equations for $\boldsymbol{\lambda}\left(t_{f}\right)$ (Eq. S24), and Eq. S28 becomes

$$
\left(\begin{array}{llllllllll}
\mathbf{D}_{11}^{0} & \mathbf{D}_{12}^{0} & \mathbf{D}_{13}^{0} & \mathbf{D}_{14}^{0} & \mathbf{D}_{15}^{0} & \mathbf{D}_{16}^{0} & \mathbf{D}_{17}^{0} & \mathbf{D}_{18}^{0} & 0 & 0 \\
\mathbf{D}_{21}^{0} & \mathbf{D}_{22}^{0} & \mathbf{D}_{23}^{0} & \mathbf{D}_{24}^{0} & \mathbf{D}_{25}^{0} & \mathbf{D}_{26}^{0} & \mathbf{D}_{27}^{0} & \mathbf{D}_{28}^{0} & 0 & 0 \\
\mathbf{D}_{31}^{0} & \mathbf{D}_{32}^{0} & \mathbf{D}_{33}^{0} & \mathbf{D}_{34}^{0} & \mathbf{D}_{35}^{0} & \mathbf{D}_{36}^{0} & \mathbf{D}_{37}^{0} & \mathbf{D}_{38}^{0} & 0 & 0 \\
\mathbf{D}_{41}^{0} & \mathbf{D}_{42}^{0} & \mathbf{D}_{43}^{0} & \mathbf{D}_{44}^{0} & \mathbf{D}_{45}^{0} & \mathbf{D}_{46}^{0} & \mathbf{D}_{47}^{0} & \mathbf{D}_{48}^{0} & 0 & 0 \\
\mathbf{D}_{11}^{f} & \mathbf{D}_{12}^{f} & \mathbf{D}_{13}^{f} & \mathbf{D}_{14}^{f} & \mathbf{D}_{15}^{f} & \mathbf{D}_{16}^{f} & \mathbf{D}_{17}^{f} & \mathbf{D}_{18}^{f} & 0 & 0 \\
\mathbf{D}_{21}^{f} & \mathbf{D}_{22}^{f} & \mathbf{D}_{23}^{f} & \mathbf{D}_{24}^{f} & \mathbf{D}_{25}^{f} & \mathbf{D}_{26}^{f} & \mathbf{D}_{27}^{f} & \mathbf{D}_{18}^{f} & 0 & 0 \\
\mathbf{D}_{51}^{f} & \mathbf{D}_{52}^{f} & \mathbf{D}_{53}^{f} & \mathbf{D}_{54}^{f} & \mathbf{D}_{55}^{f} & \mathbf{D}_{56}^{f} & \mathbf{D}_{57}^{f} & \mathbf{D}_{58}^{f} & -1 & 0 \\
\mathbf{D}_{61}^{f} & \mathbf{D}_{62}^{f} & \mathbf{D}_{63}^{f} & \mathbf{D}_{64}^{f} & \mathbf{D}_{65}^{f} & \mathbf{D}_{66}^{f} & \mathbf{D}_{67}^{f} & \mathbf{D}_{68}^{f} & 0 & -1 \\
\mathbf{D}_{71}^{f} & \mathbf{D}_{72}^{f} & \mathbf{D}_{73}^{f} & \mathbf{D}_{74}^{f} & \mathbf{D}_{75}^{f} & \mathbf{D}_{76}^{f} & \mathbf{D}_{77}^{f} & \mathbf{D}_{78}^{f} & 0 & 0 \\
\mathbf{D}_{81}^{f} & \mathbf{D}_{82}^{f} & \mathbf{D}_{83}^{f} & \mathbf{D}_{84}^{f} & \mathbf{D}_{85}^{f} & \mathbf{D}_{86}^{f} & \mathbf{D}_{87}^{f} & \mathbf{D}_{88}^{f} & 0 & 0
\end{array}\right)\left(\begin{array}{c}
\mathcal{C}_{1} \\
\mathcal{C}_{2} \\
\mathcal{C}_{3} \\
\mathcal{C}_{4} \\
\mathcal{C}_{5} \\
\mathcal{C}_{6} \\
\mathcal{C}_{7} \\
\mathcal{C}_{8} \\
\nu_{2} \\
\nu_{3}
\end{array}\right)=\left(\begin{array}{c}
x_{2}^{0} \\
x_{3}^{0} \\
x_{4}^{0} \\
x_{5}^{0} \\
x_{2}^{f} \\
x_{3}^{f} \\
0 \\
0 \\
0 \\
0
\end{array}\right)
$$

For the case of partial constraints on position, there are 9 unknowns $\left(8\right.$ in $\left.\mathcal{C}, \nu_{2}\right)$. We get 4 equations for $\boldsymbol{x}_{0}$ (Eq. S18), 1 equation for $\boldsymbol{x}_{f}$ (Eq. S25), 4 equations for $\boldsymbol{\lambda}\left(t_{f}\right)$ (Eq. S26), and Eq. $\mathbf{S} 28$ becomes 


$$
\left(\begin{array}{lllllllll}
\mathbf{D}_{11}^{0} & \mathbf{D}_{12}^{0} & \mathbf{D}_{13}^{0} & \mathbf{D}_{14}^{0} & \mathbf{D}_{15}^{0} & \mathbf{D}_{16}^{0} & \mathbf{D}_{17}^{0} & \mathbf{D}_{18}^{0} & 0 \\
\mathbf{D}_{21}^{0} & \mathbf{D}_{22}^{0} & \mathbf{D}_{23}^{0} & \mathbf{D}_{24}^{0} & \mathbf{D}_{25}^{0} & \mathbf{D}_{26}^{0} & \mathbf{D}_{27}^{0} & \mathbf{D}_{28}^{0} & 0 \\
\mathbf{D}_{31}^{0} & \mathbf{D}_{32}^{0} & \mathbf{D}_{33}^{0} & \mathbf{D}_{34}^{0} & \mathbf{D}_{35}^{0} & \mathbf{D}_{36}^{0} & \mathbf{D}_{37}^{0} & \mathbf{D}_{38}^{0} & 0 \\
\mathbf{D}_{41}^{0} & \mathbf{D}_{42}^{0} & \mathbf{D}_{43}^{0} & \mathbf{D}_{44}^{0} & \mathbf{D}_{45}^{0} & \mathbf{D}_{46}^{0} & \mathbf{D}_{47}^{0} & \mathbf{D}_{48}^{0} & 0 \\
\mathbf{D}_{11}^{f} & \mathbf{D}_{12}^{f} & \mathbf{D}_{13}^{f} & \mathbf{D}_{14}^{f} & \mathbf{D}_{15}^{f} & \mathbf{D}_{16}^{f} & \mathbf{D}_{17}^{f} & \mathbf{D}_{18}^{f} & 0 \\
\mathbf{D}_{51}^{f} & \mathbf{D}_{52}^{f} & \mathbf{D}_{53}^{f} & \mathbf{D}_{54}^{f} & \mathbf{D}_{55}^{f} & \mathbf{D}_{56}^{f} & \mathbf{D}_{57}^{f} & \mathbf{D}_{58}^{f} & -1 \\
\mathbf{D}_{61}^{f} & \mathbf{D}_{62}^{f} & \mathbf{D}_{63}^{f} & \mathbf{D}_{64}^{f} & \mathbf{D}_{65}^{f} & \mathbf{D}_{66}^{f} & \mathbf{D}_{67}^{f} & \mathbf{D}_{68}^{f} & 0 \\
\mathbf{D}_{71}^{f} & \mathbf{D}_{72}^{f} & \mathbf{D}_{73}^{f} & \mathbf{D}_{74}^{f} & \mathbf{D}_{75}^{f} & \mathbf{D}_{76}^{f} & \mathbf{D}_{77}^{f} & \mathbf{D}_{78}^{f} & 0 \\
\mathbf{D}_{81}^{f} & \mathbf{D}_{82}^{f} & \mathbf{D}_{83}^{f} & \mathbf{D}_{84}^{f} & \mathbf{D}_{85}^{f} & \mathbf{D}_{86}^{f} & \mathbf{D}_{87}^{f} & \mathbf{D}_{88}^{f} & 0
\end{array}\right)\left(\begin{array}{c}
\mathcal{C}_{1} \\
\mathcal{C}_{2} \\
\mathcal{C}_{3} \\
\mathcal{C}_{4} \\
\mathcal{C}_{5} \\
\mathcal{C}_{6} \\
\mathcal{C}_{7} \\
\mathcal{C}_{8} \\
\nu_{2}
\end{array}\right)=\left(\begin{array}{c}
x_{2}^{0} \\
x_{3}^{0} \\
x_{4}^{0} \\
x_{5}^{0} \\
x_{2}^{f} \\
0 \\
0 \\
0 \\
0
\end{array}\right)
$$

\section{References}

Bryson A (1999) Dynamic Optimization. Englewood Cliffs, NJ: Prentice-Hall. 